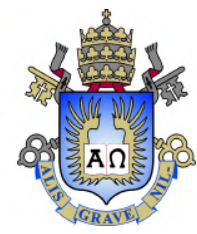

Vitor Heitor Cardoso Cunha

\title{
Breakup dynamics of thin liquid sheets with viscous interfaces
}

Dissertation presented to the Programa de Pós-graduação em Engenharia Mecânica, do Departamento de Engenharia Mecânica da PUC-Rio in partial fulfillment of the requirements for the degree of Mestre em Engenharia Mecânica.

Advisor : Prof. Márcio da Silveira Carvalho

Co-advisor: Dr. Sergio Santiago Ribeiro 


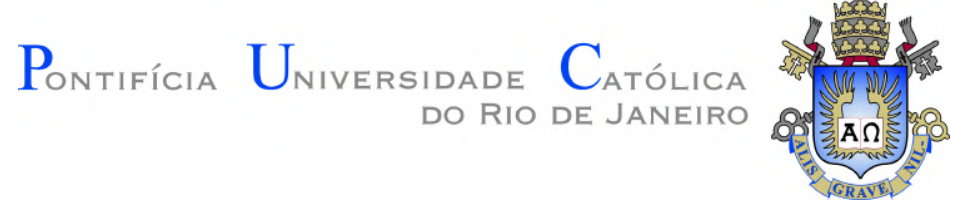

Vitor Heitor Cardoso Cunha

Breakup dynamics of thin liquid sheets with

viscous interfaces

Dissertation presented to the Programa de Pós-graduação em Engenharia Mecânica da PUC-Rio in partial fulfillment of the requirements for the degree of Mestre em Engenharia Mecânica. Approved by the Examination Committee:

Prof. Márcio da Silveira Carvalho

Advisor

Departamento de Engenharia Mecânica - PUC-Rio

Dr. Sergio Santiago Ribeiro

Co-advisor

Departamento de Engenharia Mecânica - PUC-Rio

Prof. Monica Naccache

Departamento de Engenharia Mecânica - PUC-Rio

Prof. Roney Leon Thompson

UFRJ

Rio de Janeiro, September 28th, 2021 
All rights reserved.

\section{Vitor Heitor Cardoso Cunha}

Vitor Heitor Cardoso Cunha received the diploma in Mechanical Engineering from the Department of Mechanical Engineering of the Pontifícia Universidade Católica do Rio de Janeiro (PUC-Rio). Afterwards, he began his master's degree at the Laboratory of Microhydrodynamics and Flow in Porous Media of the Department of Mechanical Engineering of PUC-Rio under the supervision of prof. Marcio Carvalho.

Bibliographic data

Cunha, V. H. C.

Breakup dynamics of thin liquid sheets with viscous interfaces / Vitor Heitor Cardoso Cunha; advisor: Márcio da Silveira Carvalho; co-advisor: Sergio Santiago Ribeiro. - 2021.

$84 \mathrm{f}$ : il. color. ; $30 \mathrm{~cm}$

Dissertação (mestrado) - Pontifícia Universidade Católica do Rio de Janeiro, Departamento de Engenharia Mecânica, 2021.

Inclui bibliografia

1. Engenharia Mecânica - Teses. 2. Filmes finos. 3. Reologia Interfacial. 4. Interfaces Viscosas. 5. BoussinesqScriven. I. Carvalho, M. S.. II. Ribeiro, S. S.. III. Pontifícia Universidade Católica do Rio de Janeiro. Departamento de Engenharia Mecânica. IV. Título. 


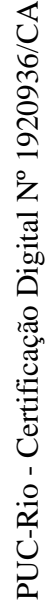

To the reader. 


\section{Acknowledgments}

The two years of development of this work coincided with the beginning

of the Covid-19 pandemic. Over the course of the research, the difficulties arising from the limitation in the exchange between employees due to social distancing became relevant. Therefore, I am immensely grateful for all who helped me with the development of this work, despite the challenges of social limitation.

The conclusion of this research is the result of the collaboration of my advisors, friends, family and the online scientific community. To my friends Rodrigo Castello Branco, Mateus Aguiar, Murilo Oliveira, Rafael Duarte, Vinicius Mattoso, Marcelo Coelho, Daniele Dias, Rafael Ferrão and Andrea Vanessa, I am very grateful for all the emotional and intellectual support you have provided me during this stage of my life . I can't think of words to describe the gratitude I feel to be part of such a rich and intense core of friends.

I am immensely grateful for the assistance provided by my partner Mariana Valente. She provided me with support that I had never experienced before and showed me that being resilient to activities you believe in is a learned trait. Thank you for motivating me to never give up, to always pursue my dreams and not be afraid of the uncertainties that come with life.

To my brothers Vinicius Manoel and André Luiz I owe all my curiosity. The passion for applied computing and linguistics is contagious and expands the way we understand reality. Thank you so much for teaching me so much about so much that you know. To my mother, Vera Lucia, I thank you in such a profound way that I can only express myself by hugging you. To my father, Roberto Cunha, you have inspired me since I was a child and contributed directly to my professional training.

To my friend Natalia Temistocles I would like to say that you are brilliant. Thank you for being someone who listens and doesn't judge. Thank you for all our studies in philosophy, logic and art together. Thanks to my dear friend of decades Brian Ripper for his absurd intensity and ability to see beyond the ordinary. Thanks also to my friend Brad Ripper who provided me insights about biology, life and several other subjects. You all have an important role in my personal and professional development.

To all fellow researchers from the Laboratory of Microhydrodynamics and Flow in Porous Media (LMMP/PUC-Rio) for their welcome, moments of relaxation and teaching. In special to Paula Reis, Monique Dali, Nicolle Lima and Rafael Chalhub for all the content-rich interactions. I am extremely grateful for the opportunity to be part of such a diverse and competent group. 
To my co-advisor Sergio Ribeiro I would like to say that you inspire me to be a more curious engineer. His way of evaluating the challenges ahead of us taught me the importance of small and consistent steps. You are a great friend who taught me a lot of what you know.

I thank my advisor Márcio Carvalho for the opportunity, stimulus, exchanges and partnership to carry out this work. I had the opportunity to be guided by one of the smartest and most cunning professionals I have ever met. Still waiting for a new opportunity to sing Creedence Clearwater Revival at the Karaoke.

At last, I thank the financial support given by the Brazilian Research Council (CNPq) and PUC-Rio, without which this work could not have been accomplished. This study was financed in part by the Coordenação de Aperfeiçoamento de Pessoal de Nível Superior - Brasil (CAPES) - Finance Code 001. 


\section{Abstract}

Cunha, V. H. C.; Carvalho, M. S. (Advisor); Ribeiro, S. S. (Co-Advisor). Breakup dynamics of thin liquid sheets with viscous interfaces. Rio de Janeiro, 2021. 84p. Dissertação de Mestrado - Departamento de Engenharia Mecânica, Pontifícia Universidade Católica do Rio de Janeiro.

Thin liquid films play a big role in many real-life applications and are of indisputable interest to scientific and industrial researchers. Evidence of thin films are observed in nature in large scales such as snow avalanches in the mountains, lava flows on volcanoes and landslides, and in small scales such as the pulmonary airways and the eye surface. They are also widespread in many industrial applications, ranging from high-resistance thin film resistors, atomization, soft-lithography methods and several coating techniques such as dip, roll, slot, spin and curtain coating. Understanding the physical mechanisms contributing to the stability of thin liquid films is a challenging problem, as thin films' flows present a fluid-fluid interface which is free to deform. The interface is bounded between two liquids or a liquid and a gas, typically having its own dynamic properties from which interfacial tension effects and complex interfacial rheological behavior arises. Instability is usually driven by long-range intermolecular forces, also known as van der Waals attractions, and may result in the rupture of the layer. Numerical investigation is often used to understand the breakup dynamics of thin liquid sheets by addressing the evolution of the film thickness using either asymptotic derivations of the lubrication theory or interface tracking techniques. In this work, a computational investigation of the breakup dynamics of a stationary thin liquid sheet bounded by a passive gas with a viscous interface is presented. The Arbitrary Lagrangian-Eulerian method (ALE) is used to track the interface position. The rheological behavior of the viscous interface is modeled by the Boussinesq-Scriven constitutive law, and the numerical solution is obtained through finite element approximation. The results show that thin liquid film stability is influenced both by surface rheology and disjoining effects and that the viscous character of the interface delays the sheet breakup, leading to more stable films.

\section{Keywords}

Thin liquid films; Interfacial rheology; Viscous interfaces; BoussinesqScriven. 


\section{Resumo}

Cunha, V. H. C.; Carvalho, M. S.; Ribeiro, S. S.. Ruptura de filmes finos líquidos com interfaces viscosas. Rio de Janeiro, 2021. 84p. Dissertação de Mestrado - Departamento de Engenharia Mecânica, Pontifícia Universidade Católica do Rio de Janeiro.

Filmes finos líquidos desempenham um grande papel em diversas aplicações cotidianas e são de interesse indiscutível para pesquisadores científicos e industriais. Evidências de filmes finos são observadas na natureza em grandes escalas, como avalanches de neve nas montanhas, escoamento de lava em vulcões e deslizamentos de terra, e em pequenas escalas, como nas vias respiratórias pulmonares e na superfície dos olhos. Eles também são estão presentes em muitas aplicações industriais, variando de resistores de filme fino de alta resistência, atomização, métodos de litografia e várias técnicas de revestimento. Entender os mecanismos que contribuem para a estabilidade de filmes finos líquidos é um problema desafiador, pois o escoamento de filmes finos apresenta uma interface fluido-fluido livre para deformar. A instabilidade de um filme fino é geralmente impulsionada por forças intermoleculares de longo alcance, também conhecidas como atrações de van der Waals, e resultam na ruptura do filme. Investigações numéricas são frequentemente usadas para entender a dinâmica de ruptura de filmes líquidos finos, abordando a evolução da espessura do filme usando derivações assintóticas da teoria da lubrificação ou técnicas de rastreamento de interface. Neste trabalho, uma investigação computacional da dinâmica de ruptura de um filme fino líquido estacionário com uma interface viscosa é apresentada. O método Arbitrary Lagrangian-Eulerian (ALE) é usado para rastrear a posição da interface. O comportamento reológico da interface viscosa é modelado pela lei constitutiva de Boussinesq-Scriven, e a solução numérica é obtida através da aproximação de elementos finitos. Os resultados mostram que a estabilidade do filme líquido fino é influenciada tanto pela reologia da superfície quanto pela atração intermolecular e que o caráter viscoso da interface retarda a quebra da folha, levando a filmes mais estáveis.

\section{Palavras-chave}

Filmes finos; Reologia Interfacial; Interfaces Viscosas; BoussinesqScriven. 


\section{Table of contents}

1 Introduction $\quad 13$

1.1 Motivation 13

$\begin{array}{lll}1.2 & \text { Research objectives } & 18\end{array}$

$\begin{array}{llr}1.3 & \text { Outline } & 18\end{array}$

$2 \quad$ Literature review and background $\quad 19$

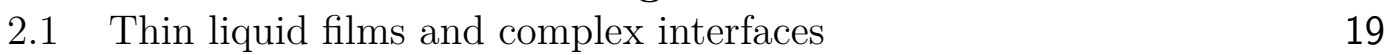

$\begin{array}{lll}2.2 & \text { Interface tracking methods } & 27\end{array}$

$3 \quad$ Problem formulation 33

3.1 Governing equations $\quad 34$

3.1.1 Bulk dynamics 34

$\begin{array}{lll}3.1 .2 & \text { Interface dynamics } & 35\end{array}$

$\begin{array}{lll}3.1 .3 & \text { Boundary conditions } & 36\end{array}$

$\begin{array}{lll}3.2 & \text { Dimensionless parameters } & 37\end{array}$

4 Numerical implementation $\quad 40$

4.1 The finite element method $\quad 41$

4.2 Weak form and residual equation 43

4.3 Arbitrary Lagrangian-Eulerian method $\quad 47$

$\begin{array}{lll}4.4 & \text { Numerical procedure } & 49\end{array}$

$5 \quad$ Results and discussion $\quad 52$

5.1 Mesh convergence test $\quad 52$

5.2 Simple interface $\quad 54$

5.2.1 Literature comparison $\quad 54$

5.2.2 Thin film rupture $\quad 60$

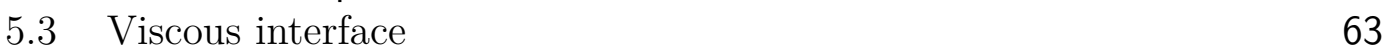

$\begin{array}{lll}\text { 5.3.1 Amplitude growth } & 64\end{array}$

5.3.2 Velocity and pressure fields 66

6 Conclusion $\quad 71$

$\begin{array}{lll}6.1 & \text { Future works } & 72\end{array}$

$\begin{array}{lll}7 & \text { Bibliography } & 74\end{array}$ 


\section{List of algorithms}

1

procedure51
Newton-Raphson method472 


\section{List of Abreviations}

DES - Dry Eye Syndrome

OTFT - Organic Thin Film Transistor

RFID - Radio Frequency IDentification

OPC - Organic Photovoltaic Cell

PSC - Perovskite Solar Cell

PCE - Power Conversion Efficiency

DPPC - Dipalmitoylphosphatidylcoline

MAC - Marker and Cell

SPH - Smooth Particle Hydrodynamics

LS - Level-Set

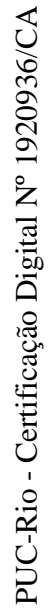

VOF - Volume of Fluid

FSI - Fluid Structure Interaction

ALE - Arbitrary Lagrangian-Eulerian

FEniCS - Finite Element Computational Software

CFL - Courant-Friedrichs-Lewy 
"Hat man sein warum des Lebens, so verträgt man sich fast mit jedem wie." (If you have your why of life, you get along with almost every how.)

Friedrich Nietzsche, Götzen-Dämmerung; oder, Wie man mit dem Hammer philosophirt. 


\section{Introduction}

\section{1}

\section{Motivation}

The dynamics of thin liquid films have fascinated scientists over many decades, presenting several interesting multiphysics aspects $[1,2]$. They are central to the study of numerous areas of engineering, geophysics, and biophysics, and can occur over a wide range of length scales in nature, such as lava flows and snow avalanches [3], tear-film thinning and tear breakup [4] and the liquid lining protecting the pulmonary airways [5]. Many industrial processes also show a thin film configuration, such as solid state thin film lithiumion battery systems [6], atomic layer deposition techniques [7], high-resistance thin film resistors, atomization, soft-lithography methods, and several coating techniques such as dip, roll, slot, spin and curtain coating $[8,9]$. Thin liquid films can generate a host of fascinating behaviors, as they present a fluid-fluid interface that is free to deform. The interface is bounded between two liquids or a liquid and a gas, where each particular interface typically presents its own dynamic properties from which surface tension effects and complex interfacial rheological behavior may arise. Figure 1.1a (image from [10]) and 1.1b (image from [11]) illustrate the presence of naturally occurring thin film phenomena, by means of a liquid film covering the human eye and lava flow, respectively, whereas Figure 1.1c (image from [12]) pictures a printed organic circuit for low voltage applications of slender aspect ratio.

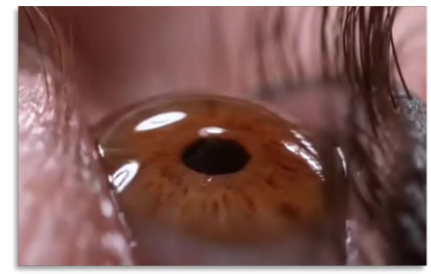

(a)

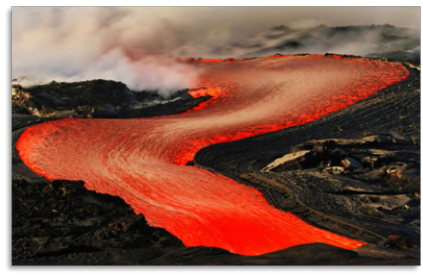

(b)

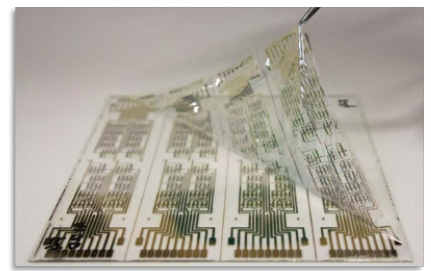

(c)

Figure 1.1: (a) Close look at the human eye; (b) Lava flow in Hawaii; Ultrathin printed organic logic circuit for low-voltage wearable sensor applications.

In nature, the rupture of thin liquid films bounded by a solid substrate and a free surface is one of the core mechanisms in common health conditions, such as the dry eye syndrome (DES), in which instabilities lead the tear film to quickly breakup, leading to a poor coat of the eye surface. The tear film 
is typically considered a three-layered film with a very thin lipid layer on its free surface, a relatively thick aqueous layer and a bound mucin layer on the bottom, as depicted in Figure 1.2a (adapted from [13]). DES is widely accepted to be caused by hyperosmolarity and tear film instability [4]. Another occurrence of pathologies due to thin film instability is the respiratory distress syndrome, where the lungs of prematurely born infants are not sufficiently mature to produce adequate quantities of pulmonary surfactant, giving rise to respiratory difficulties associated with airway closure, pulmonary edema and mechanical damage of the airway linings [5]. Lung surfactants flow up the terminal airways through a thin aqueous protective layer and reduces the formation of liquid plugs that can obstruct terminal airways at endexpiration, thus supporting clearance of inhaled particulates and pathogens. These surfactant monolayers are illustrated in Figure 1.2b (adapted from [14]). The mechanisms through which lung surfactants lower the surface tension inside our lungs increase the pulmonary compliance, thus facilitating the breathing process [15]. In the absence of naturally occurring surfactants, artificial surfactant replacements can be introduced as a clinical treatment. In such flows, the decrease of the film thickness followed by the breakup is a phenomenon known as dewetting [16]. Understanding the stability of the thin surfactant films through fluid mechanical processes may lead to a better understanding of the proper mechanisms of which clinical interventions rely. The role of interfacial rheology in these pathologies has been suggested to be of great importance, along with adsorption/desorption dynamics [17].

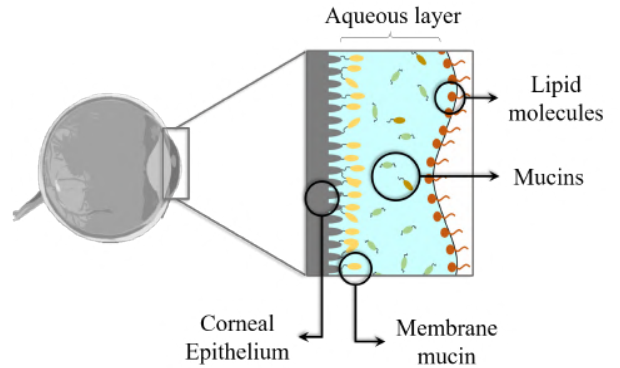

(a)

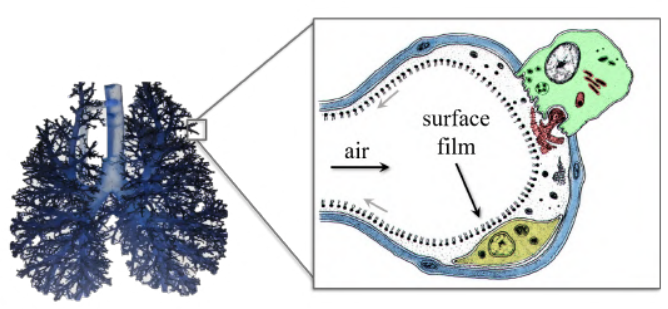

(b)

Figure 1.2: Sketches of surface phenomena: (a) the composition of the precorneal tear film and of (b) naturally occurring surfactant film covering the lung surfaces.

Thin aqueous sheets are also used to access biochemical insights of liquid environments with photon spectroscopy techniques. Microfluidic gas-dynamic nozzles with small orifice diameter enables the generation of a chain of multiple mutually orthogonal ultrathin liquid sheets in a vacuum chamber, which are 
then used in time-dependent experiments of soft-x-ray spectroscopy [18]. The inferred information obtained from analysis of spectroscopy techniques may showcase key insights into the local molecular interactions in liquids solutions, but the necessary probe samples often rely on a limited thickness from sub micrometer to a few micrometers due to infrared and soft x-ray spectroscopy detection scheme and strong absorption of the aqueous phase.

The use of gas-dynamic forces from focused gas flow through microfluidic nozzles to generate sub-micron thick free liquid sheets allow the reduction of the liquid flow rate by an order of magnitude relative to other techniques such as the colliding liquid jets. This results in much thinner sheets that can readily transmit infrared radiation and soft x-rays. In these microfluidic configurations, the liquid flow is kept constant and the gas flow is increased incrementally, and a gradual spread of the liquid into a sheet is achieved as the speed of the colliding gas jet is increased. The gas also protects the ultrathin free liquid sheet from freezing near the nozzle in the case of vacuum operation due to evaporative cooling, as the progressive reduction of the sheet thickness occur. The use of ultrathin liquid sheets is thus an attractive alternative for soft-xray absorption spectroscopy. Koralek et al. [19] demonstrated the generation of ultrathin free liquid sheets of de-ionized water with thickness tunable down to 10 's of nanometers that can operate stably in vacuum for days with microfluidic nozzles. Such ultrathin free liquid sheets correspond to fewer than 100 water molecules thick, and are potentially transformative for infrared, soft x-ray and electron spectroscopies.

Industrial applications such as aerosol droplet generation [20], inkjet printing [21] and monodisperse emulsions fabrication [22] rely on the rupture of thin liquid sheets in their procedure. However, the breakup process of thin films can be undesirable in applications such as slot die and curtain coating [23]. Figure 1.3 (adapted from [24, 25]) portrays an atomization procedure on the left and a curtain coating process on the right. The sheet rupture is required to produce dispersed fluid droplets, whereas in curtain coating, the breakup of the liquid sheet ruins the process. Hence, thin films are key elements in the manufacturing of several electronic devices, such as nanoscale lubricant coating of head-disc interfaces in hard disk drives and nextgeneration ultrathin electronics [26, 12]. Regarding the latter (Figure 1.1c), the fabrication of organic thin film transistor (OTFT) technology lead to several performance enhancements enabled its use in applications such as practical wearable devices, flexible displays [27] and radio frequency identification (RFID) wireless tags [28]. These organic electronic solutions enable thin film organic photovoltaic cells (OPCs) as a potential technology in the generation 
of electric energy, driving the scientific community to research for fast and inexpensive solutions for the manufacturing of OPCs [23].

Desirable rupture

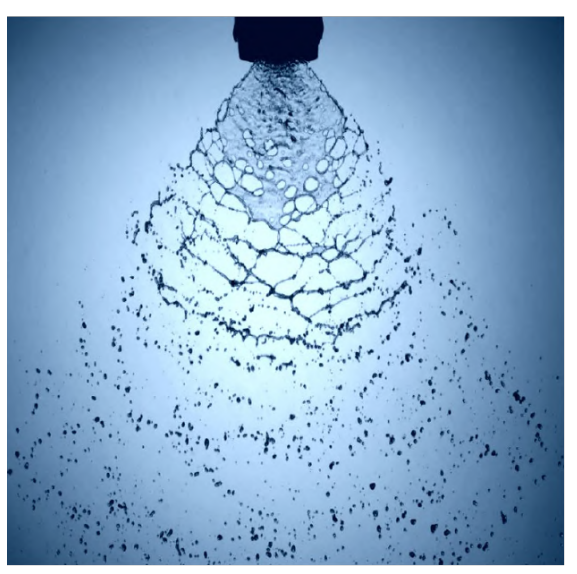

Undesirable rupture

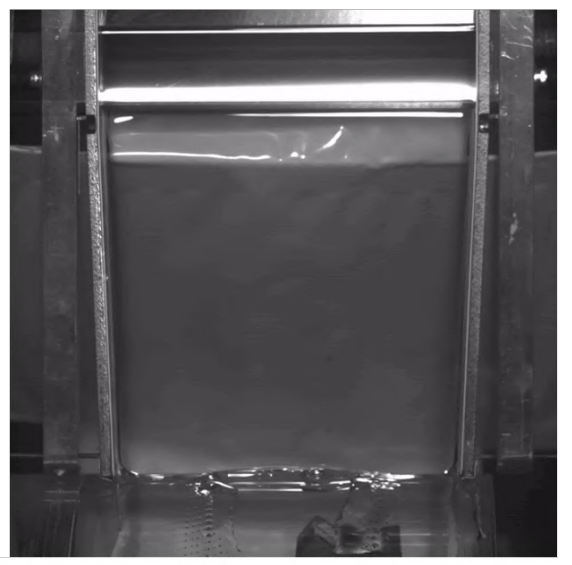

Figure 1.3: Portraits of liquid atomization process and curtain coating, from left to right.

Recently, perovskite solar cells (PSCs) are being accepted as auspicious candidates to supply electricity by the scientific and industrial community, despite the challenges in scaling their fabrication to meet current energy demands. PSCs have a thin electron transport layer attached directly to a perovskite layer, which can be arranged in ways to reduce photocurrent voltage hysteresis phenomenon and thus resulting in high-efficiency performance solar cells [29]. The use of methylammonium lead trihalides (MAPbX3) perovskites as inorganic sensitizers in solar cells was introduced by Kojima et al. [30] and improved by Im et al. [31], reaching an overall power conversion efficiency (PCE) of 6.5\%. Further developments of PSCs relied on the replacement of liquid electrolytes by a solid hole-conducting material such as titanium dioxide films with thickness ranging from 2 to $0.2 \mu \mathrm{m}$, leading to an overall PCE of $25.2 \%$ on a laboratory scale [32]. Moreover, there are several challenges in upscaling the assembly of PSCs to larger areas, as the manufacturing of these solar cells is done using spin coating techniques.

The thin film configuration is also presented in all-solid-state thinfilm batteries, which are part of the next generation of batteries due to their high power and energy densities and long battery lifetimes [33]. The development of preliminary studies of all-solid-state thin-film batteries is driven by the simplification on the battery assembly, size reduction and performance requirements of electronic devices [6]. Each thin film battery component is deposited by a coating process in which previously deposited layers are stable for subsequent film depositions and thermal treatments, with 
an assembled battery ranging from $100 \mu \mathrm{m}$ down to $5 \mu \mathrm{m}$ of thickness. In addition, the use of ultrathin layer-by-layer assembly has become a versatile nanocoating on polypropylene separator to suppress deterioration of lithiumsulfur batteries from polysulfides dissolution [34]. Applications such as thinfilm photovoltaic cells or all-solid-state thin film batteries rely on smooth and uniform films, which on a solid substrate can become unstable due to intermolecular forces and lead to malfunction of such applications.

Liquid-liquid and liquid-gas interfacial forces play a key role in the stability of thin liquid sheets. Depending on the problem scale, different behavior must be considered. As examples are interfacial viscosity, longrange intermolecular attraction between the interface molecules and diffusive transport of materials across the interface [35, 36, 37]. In most practical applications, the instability of a thin liquid sheet is driven by long-range molecular forces due to van der Waals attractions, whereas capillary and viscous forces have a stabilizing effect [38, 9]. A liquid film rupture occurs when it is sufficiently thin, in which the long-range intermolecular forces become dominant [1]. Also, the rupture of free films driven by van der Waals attractions is closely related mathematically to the problem of surface-tensiondriven pinch-off of an axisymmetric fluid thread, in which the dynamics of rupture in both phenomena are often described by two set of differential equations governing mass and momentum conservation, with the geometry of the phenomena affecting the form of these equations [39].

Considering the rupture dynamics, the ultimate lifetime of liquid films is determined by two processes: thinning and growth of surface fluctuations, and the rupture gives rise to a finite time singularity in the governing equations. For both films supported by a substrate and free films, the thinning process is produced by drainage of the film phase under the influence of gravity and capillary suction, and the action of the long-range attractive forces increases as the film thickness decreases [40, 41].

In both nature and industry, these surface fluctuations are often stabilized by the presence of surface-active agents (surfactants) that can extend the life span of thin films in a considerable manner. The response of the interface in these cases is strongly dependent on the chemical composition of the surfactants, which may reduce the drainage rate of the thin films according to the mechanisms by which the surface-active agents operate [42]. The interaction within the interface due to a significant microstructure lead to extra and deviatoric interfacial stresses and possibly rheologically complex behavior of the interface [37]. These complex interfaces can yield viscous and viscoelastic responses to deformations and may reduce the mobility of the interface. 


\section{2 \\ Research objectives}

The study of thin film dynamics may lead to insights for many technological breakthroughs in areas such as photovoltaic systems, thin sheet solid state batteries, lithography, additive manufacturing and ultrathin polymeric coating. The underlying rupture mechanisms of these flows are encompassed in a complex interplay between capillarity, hydrodynamics and interfacial stresses and are of utmost importance for quality control, reliability and reproducibility of industrial processes. Therefore, in this work, the influence of interface rheology on the dynamics of stationary free thin liquid films is studied. We aim at understanding the mechanisms by which interfacial stresses of a viscous interface delay the breakup time of a stationary free thin liquid sheet. The main objective of this dissertation is to analyze and compare the rupture dynamics of free liquid films with inviscid and viscous surfaces.

\section{3}

Outline

This dissertation is organized in the following chapters: Chapter 2 presents a literature review of thin liquid sheets with complex interfaces and interface tracking methods. We provide a multidisciplinar overview of the literature to account for foundations of thin film studies.

In Chapter 3, we describe the complete mathematical formulation of both simple and viscous cases. We review the physical aspects of the problem and state the assumptions used in this manuscript.

Chapter 4 presents the numerical methods used in this investigation. More specifically, we present a theoretical introduction on the tools used herein. Chapter 5 illustrates the results of our investigation along with a discussion. Lastly, Chapter 6 provides some thoughts on future works. 


\section{2}

\section{Literature review and background}

\section{1}

\section{Thin liquid films and complex interfaces}

As previously mentioned, the growth of surface fluctuations can be spontaneous or induced, driven by long range intermolecular attractions. Derjaguin and Obuchov [43] introduced the concept of a disjoining pressure, which is defined as the sum of the equilibrium intermolecular forces acting between two opposing surfaces of a liquid sheet. The stability of thin liquid films is strongly influenced by the magnitude of the film's disjoining pressure, whose first experimental determinations about its molecular components confirmed its attractive nature, as published by Derjaguin and Abrikosova [44]. These intermolecular forces arise from fluctuations in the electromagnetic field present in the medium, thus resulting in changes on intensive thermodynamic properties. In view of the above, Lifshitz [45] developed a rigorous macroscopic theory for the interaction of bodies whose surfaces are brought within a small distance.

Such intermolecular forces are often referred as van der Waals forces due to their resemblance with the van der Waals forces of attraction between molecules at large distances. Thus, assuming that the interaction between the material molecules obey the van der Waals law, an arbitrarily thick material will present small contributions of the long wave fluctuations relative to the free energy of the body. However, in thin films, the chemical potential $\mu$ of the same material will express a dependence on the film thickness $h$ of $1 / h^{3}$, leading the appearance of van der Waals forces of mutual attraction between surfaces [46]. Later on, Dzyaloshinskii et al. [47] derived a general formulation for the thermodynamic quantities such as chemical potential of thin liquid films under the action of van der Waals forces.

The stability of free liquid sheets is strongly influenced by the interplay between capillary, intermolecular forces and interfacial stresses. Pressure gradients caused by capillary forces lead to flows that may yield additional hydrodynamic forces that can influence the movement of the interface. Concerning flows of high interface area, the interfaces between the liquid and its surroundings were first characterized by their surface energies, in which surface energy represents the minimum amount of work required to create that interface. 
Thus, the interfacial tension between two phases is determined by the measure of the interfacial free energy per unit area. The capillary number $C a$ is commonly defined to describe the action of capillarity and hydrodynamics on the system, which is taken as the ratio between viscous forces and interfacial tension forces

$$
C a=\frac{\mu U}{\sigma_{\alpha \beta}}
$$

Where $\mu$ is the dynamic viscosity, $U$ is a characteristic flow velocity and $\sigma_{\alpha \beta}$ is the constant interfacial tension between the phases.

Taylor [48] was the first to analyze the stability of free liquid sheets, aiming to understand the wave propagation on the surface of free films. In his work, he identified that the surface of the free liquid sheet can exhibit two distinct dynamical modes: an antisymmetric or stretching mode where the free liquid film buckles; and a symmetric or squeezing mode, in which the opposite surfaces move towards each other, urging the possibility of film rupture, as depicted in Figure 2.1.

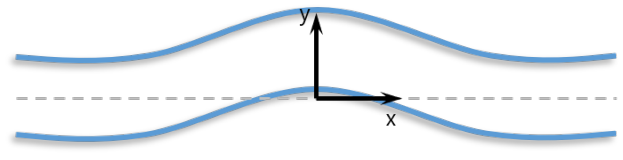

(a)

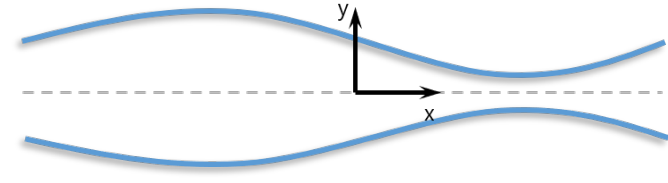

(b)

Figure 2.1: Adapted schematic of Taylor's surface dynamical modes. (a) Antisymmetric mode, characterized by the buckling of the thin liquid film; (b) Symmetric mode, in which opposite surfaces attract each other.

Later on, Brown [49] carried out an investigation to understand the behavior of a thin liquid sheet falling on a moving surface, which occurs in curtain coating process. He stated that a continuum film could be uniformly laid down on a rapidly moving surface and be industrially employed if certain conditions regarding the curtain length, flow rate and impingement velocity are respected.

Sheludko [40] observed that the amplification of spontaneous fluctuations in free liquid films are operative for sheet thickness ranging between $10 \mathrm{\eta m}$ and $100 \eta \mathrm{m}$, and that nonlinearities arise due to long range intermolecular effects. The understanding of thin film rupture was extended theoretically by the derivation of a nonlinear evolution equation for the thickness of a thin film on a solid substrate by Williams and Davis [50]. In their study, a nonlinear 
partial differential equation is solved by numerical methods, which resulted in a characteristic rupture time calculation 10 times smaller than rupture time obtained from previous linear approaches. They also proposed a nonlinear stability theory based on the long-wave nature of the thin film perturbation.

Prévost and Gallez [41] took a similar approach and investigated the nonlinear effects on the stability of free film rupture, and showed that the squeezing mode firstly reported by Taylor is the more appropriate mode to describe the mechanism of rupture of a free liquid film. Through approximations of long wavelengths, they concluded that the effect of hydrodynamic nonlinearities accelerates the rupture process due to the increase of the action of long-range attractive forces as the film thickness diminishes. Moreover, Lin et al. [51] performed a linear stability analysis of a viscous liquid sheet and showed that there are two independent modes of instability, namely sinuous and varicose modes, in the presence of ambient gas.

The long wavelength approximation was later exploited by Erneux and Davis [38], who considered the Navier-Stokes equations with an extra term comprehending the van der Waals attraction to derive asymptotically a system of governing nonlinear evolution equations for longitudinal velocity and film thickness. They applied stability analysis on the set of equations and derived a weakly non-linear stability criterion for a Newtonian fluid based on the ratio between surface tension and van der Waals forces. Ida and Miksis [52] then solved numerically the set of equations proposed by Erneux and Davis [38] and examined the dominant balances in the evolution equations using similaritytimes solutions in the temporal and spatial vicinity of rupture.

The influence of the chemical composition of a free surface has long been recognized to change its dynamics, thus asserting the importance of interfacial rheology for the understanding of complex surface phenomena [53]. These complex fluid interfaces are often characterized by the presence of amphiphilic molecules such as surfactants, proteins and particles that may induce microstructures with significant mechanical strength, along with possible thermodynamic complexities. A sketch of a microstructure formation in a liquid interface is depicted in Figure 2.2. 


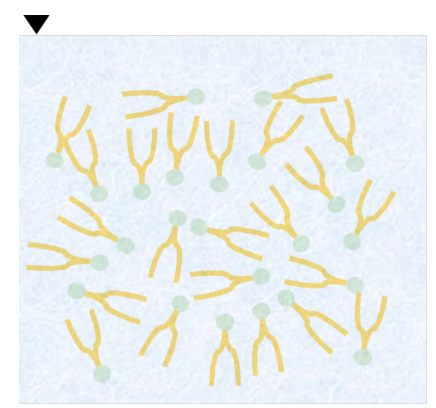

Aqueous solution

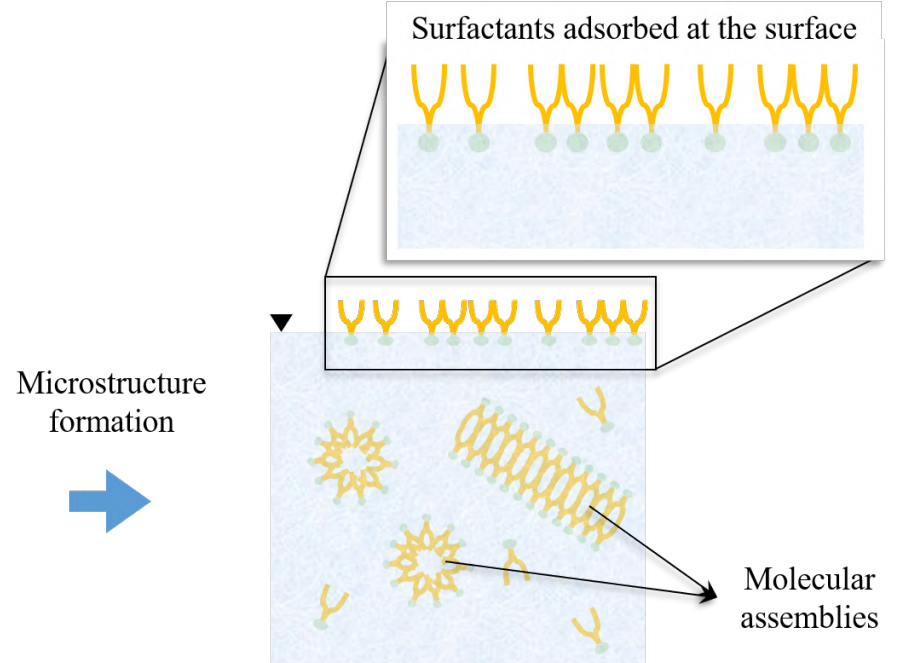

Figure 2.2: Sketch of microstructure formation in both bulk and interface regions. The presence of surfactants adsorbed on the surface yields additional mechanical resistance of the surface, thus influencing the system dynamics.

Amphiphilic molecules are often added as stabilisers to liquid mixtures and are required in the processing and functioning of many industrial applications [37]. These molecules adsorb at the interface and the process depends on the $C a$ number and the thickness of the liquid film. However, nonhomogeneous surfactant concentration and temperature differences over the interface cause a surface tension gradient $\Delta \sigma_{\alpha \beta}$, and the unbalanced force along the interface lead to the appearance of interfacial stresses known as Marangoni stresses $[53,54]$.

Thus, the presence of molecular assemblies along interfaces may influence how hydrodynamic stresses are transmitted in the system, as viscous, viscoelastic and Marangoni stresses arise from the interaction between the fluid molecules and the complex microstructures [55]. Interface diffusion and Marangoni convection opposes to surfactant concentration variations over the interface, whereas surface viscous stresses reduce the surface velocity gradients [56].

Interfacial rheology describes the functional relationship between the deformation of a complex interface, the stresses along it and the resulting flows in the adjacent fluid phases [57]. The response of these complex interfaces against deformations depends on their composition and are characterized by different rheological properties, measured during dilatation and shear deformation $[58,59]$.

Given an interfacial film, interfacial rheometry, which is the measurement of rheological properties of an interface, can be performed by both dilatational and shearing deformation of the interfaces, which are studied by dilatational 
rheology and shear rheology, respectively [60]. In dilatational rheology, one measures the variation of area while maintaining a constant shape, whereas shear rheology deals with changes in shape at constant area while considering that the interface is in thermodynamic equilibrium with the bulk solution. Distinct dynamic properties of interfaces arise for each type of deformation, such as interfacial dilatational viscosity, $\kappa_{s}$, and interfacial shear viscosity, $\mu_{s}$. In order to assure that the stress measured in interfacial rheology tests comes mainly from the interfacial behavior and not from the bulk, it is important to quantify the ratio between interfacial and bulk forces. The Boussinesq (Bo) number is defined as the ratio between the viscous forces at the interface to viscous forces in a fluid bulk of thickness $H$.

$$
B o=\frac{\kappa_{s}+\mu_{s}}{\mu H}
$$

A rheologically complex interface is often modeled through interfacial constitutive models that account the extra and deviatoric contributions to the interfacial stress from the interfacial microstructure. A general expression for the interfacial stress $\sigma_{s}$ of a complex interface is given by Eq 2-1 $[61,55]$.

$$
\sigma_{s}=\sigma\left(\Gamma_{c}, T\right) I_{s}+\sigma_{e}
$$

The term $\sigma(\Gamma, T)$ is the interfacial tension that depends on the surfactant concentration $\Gamma_{c}$ and temperature $T, I_{s}$ is a the surface unit tensor and $\sigma_{e}$ is the surface extra stress. The aforementioned approach takes into consideration the sharp-interface to account for the interfacial stress, where a zero-thickness dividing interface is positioned in between the fluid phases, yielding a quasi-2D interface. As a result of that, the bulk properties of the fluids are homogeneous up until the dividing surface and excess quantities are assigned to the dividing surface to account for any energy or mass that is not accounted for by the homogeneous bulk phases.

A general form of an equation to describe a linear dependence of extra stress $\sigma_{e}$ on rate of strain at the interface was first introduced by Boussinesq [62] and later generalized by Scriven [53] to account for the evolution of Newtonian fluids in the interfacial state, which is nowadays known as the BoussinesqScriven model:

$$
\sigma_{e}=\left(\kappa_{s}-\mu_{s}\right)\left(\nabla_{s} \cdot u\right) I_{s}+2 \mu_{s} D_{s}
$$


Here $\nabla_{s}=I_{s} \cdot \nabla$ is the surface gradient operator, $u$ is the velocity vector along the interface and $D_{s}=0.5\left(\nabla_{s} u \cdot I_{s}+I_{s} \cdot\left(\nabla_{s} u\right)^{T}\right)$ is the surface rateof-deformation tensor. The Boussinesq-Scriven model is a Newtonian model for viscous interfaces derived from a differential geometry approach in tensor form. Hence, interfacial shear and dilatational viscosities can have a significant effect on the flow behavior, and effects caused by these properties can strongly influence the dynamics of thin liquid films and other high interface materials [63]. In most practical applications, thin film stabilisation is caused by a blend of surface-active components of different sizes that yield extra viscous and viscoelastic stresses that are strongly coupled with the steric intermolecular interactions [55].

Jensen and Grotberg [64] used lubrication theory to study the spreading rate of a localized monolayer of insoluble surfactant on the surface of a thin viscous film, in the limit of weak capillary and weak surface diffusion. In their work, they describe the mechanism by which surfactant stabilizes the rupture process, and states that a non-uniform surfactant distribution along the free surface creates a surface tension gradient which then induces a shear stress at the surface of the underlying liquid, and thus a Marangoni flow in the substrate spread the surfactants across the domain. They also indicate that the presence of insoluble surfactants decreases the growth rate of the instability, but do not affect critical wavelengths, and the stabilizing influence of surfactants remains relatively weak. De Wit and Gallez [65] performed an analogous study, in which they analyzed the dynamics of free liquid films with insoluble surfactants. They derived a system of nonlinear evolution equations asymptotically from the full Navier-Stokes equations for free films and incorporated the effect of van der Waals attraction, capillary forces and Marangoni forces due to gradients of surface tension. They showed that surfactant monolayer has a stabilizing effect on the growth of the instability, and observed that the physical role of the surfactant alters the time of rupture of the free film.

Vaynblat et al [39] extended the studies on the rupture dynamics of thin liquid films by introducing two main rupture geometries, line rupture and point rupture, as portrayed in Figure 2.3. They considered a free viscous film exhibiting both simple Newtonian and more complex power-law rheology and a simple interface with vanishing viscous and elastic stresses. Moreover, they show that both line and point rupture of a free liquid film under van der Waals forces asymptote toward a self-similar regime in which the film thins according to a power law, and that the scaling exponent of film thickness has the same value in both line and point rupture. In order to further understand how the rheological properties of the liquid influence a free liquid sheet stability, 
Becerra and Carvalho [8] proceeded with an examination regarding the flow of free viscoelastic liquid sheets occurring in curtain coating processes. They showed that high extensional viscosity due to rheological behavior of polymer solutions on the flow create more stable curtains. Additionally, they pointed out that the apparent extensional viscosity of the liquids raised with the polymer concentration lead to the presence of higher normal stress that resists the growth of a hole in the curtain, which De Wit and Gallez [65] also presented in their investigation.
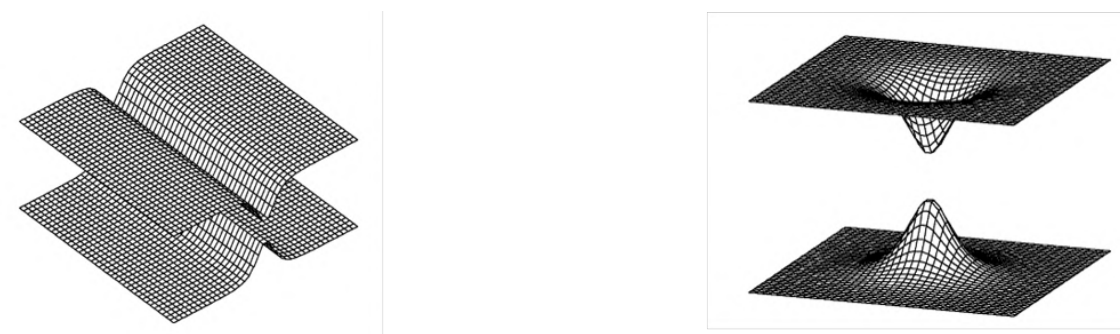

Figure 2.3: Line and point rupture geometries.

More recently, Thete et al. [66] examined the self-similar evolution of free liquid films of power law fluids towards the rupture spatial and temporal location through numerical methods. They identified two regimes of thinning depending on the power-law index $0<n \leq 1$ of the fluid, in which for $6 / 7 \leq n \leq 1$, the sheet thins and ruptures under the competition between inertial, viscous and van der Waals forces, and for $n<6 / 7$, the dominant balance of forces changes and viscous force becomes asymptotically negligible and the sheet thins and ruptures under inertial, van der Waals and surface tension forces. Later on, Thete et al. [67] have also revealed that the scaling exponents for film thickness in any power-law index have the same value regardless of whether the film undergoes line or point rupture, complementing the findings of Vaynblat et al. [39].

Furthermore, Bazzi and Carvalho [9] addressed the effect of viscoelastic properties of polymer solutions of the breakup process of a free liquid sheet with a simple interface. They studied the response of stationary Newtonian and viscoelastic thin liquid sheets to planar and axisymmetric disturbances by extending the stability criterion proposed by Erneux and Davis [38] for axisymmetric perturbations and Oldroyd-B liquids, and showed through numerical solutions that the effect of rheology slows down the perturbation growth drastically and thus slowing the sheet rupture.

The additional stresses associated with the viscoelastic behavior contribute to the decrease of the growth rate of the perturbation in a free thin liquid film, leading to substantial changes in the dynamics with respect to 
Newtonian fluids. Underhill et al. [68] presented a model to describe interfacial hydrodynamics that allows for shear-thinning response of the interface and that can be decoupled from the bulk flow in the limit of highly viscous interfaces. They also compared the results obtained with experimental measurements of dipalmitoylphosphatidylcholine (DPPC) monolayers, which is the primary constituent of lung surfactants.

Concerning tear films, Bhamla et al. [69] proposed an experimental platform to replicate the human tear film on a contact lens to investigate the influence of interfacial viscosity against the breakup and dewetting phenomena. They concluded that tear film stabilized by surface rheology and evaporation of the liquid layer result in dewetting via nucleation. Bhamla et al. [42] analyzed the drainage dynamics of surfactant-laden aqueous films with both insoluble and soluble surfactants, resulting in viscoelastic and inviscid interfaces, respectively. They showed that films are stabilized through interfacial rheology at high surface pressures and through Marangoni stresses at low surface pressures.

Moreover, aqueous films with soluble surfactants are stabilized purely through Marangoni effects and interfacial viscosity. Dey et al. [13] also analyzed the stability of tear films, as the rupture of the film that shields the ocular surface from harmful environmental factors may lead to health conditions. The tear film contains a mixture of several proteins and other biomolecules in an aqueous solution subject to the action of van der Waals forces that lead the sheet to rupture. The authors proposed a framework for modeling tear-film breakup carried out by the thin-film approximation and raised several possible mechanisms to account for the occurrence of eye diseases.

Experimental analyses also show that the rheologic characteristics of liquids strongly influence their behavior in industrial applications. Karim et al. [25] studied the effect of viscoelastic forces on the hole growth speed in a liquid curtain by high-speed visualization and showed that elastic stresses stabilize the liquid curtain by both the reduction on the retraction rim speed observed with viscoelastic solutions and by the delay on the growth rate of any disturbance that may lead a hole in the curtain. Karim et al. [70] performed a thorough analysis on the rheological behavior of dilute xanthan gum solutions and reveal that the dynamics of curtain breakup is governed by the shear viscosity at a characteristic deformation rate. They also showed that for Newtonian liquids, the stability increases as the characteristic viscosity rises. Additionally, Karim et al. [71] proposed the use of a two-layer configuration with a shear-thinning liquid as the bottom layer and a viscoelastic liquid as the top layer to delay the breakup of the liquid sheet and to reduce the 
minimum film thickness. They stated that the thickness reduction is a function of the viscoelastic stress of the top layer liquid, and reported a film thickness reduction of $60 \%$ for an aqueous solution of polyethylene oxide with $0.1 \mathrm{wt} \%$ as the viscoelastic fluid.

A growing number of emerging technologies involving the manipulation of liquid metals from millimeter to sub-micron scales, such as additive manufacturing, electronics, solar cells, plasmonic-related applications, among others, in which inertial effects play a big role on the thin film dynamics and lubrication theory cannot be used to describe the local flow close to rupture $[72,73]$. In this sense, González et al. [74] put in evidence the effects of inertia and the two-dimensional aspects of a flat liquid film extended over a solid plane and performed numerical simulations of the nonlinear Navier-Stokes equations and analyze to which extent the linear predictions can be applied for cases involving inertial and different aspect rations of the film. They found that inertia does not lead to new regions of instability, but their results show that the growth rates of the instability decrease as inertial effects are stronger.

There are few works available in the literature that discuss the effect of interfacial rheology on the rupture of thin films. Furthermore, the few works that address the topic are almost all experimental.

\section{2 \\ Interface tracking methods}

The flow of free thin liquid sheets is an intrinsic example of a multiphase flow. The temporal evolution of fluid phases distribution in a multiphase flow is often a very difficult task to predict due to their dependence on several parameters such as geometry, fluid properties and flow regime, and are commonly characterized in dispersed flows and separated flows [75]. To tackle the temporal evolution of multiphase systems in continuum mechanics, a computational mesh that covers the physical domain is often required, such as in finite elements simulations. From a computational perspective, an accurate model of multiphase flow requires proper treatment of the interaction between the phases and the liquid interface between them, along with reliable methods to track the evolution of the interface position with time.

Continuum-based fluid mechanics studies of free boundary problems involving the motion of fluid interfaces rely mainly on two approaches: a diffuse interface method with a virtual finite thickness and a sharp interface of zero thickness. A diffuse interface representation is based on a phenomenological model due to Cahn and Hilliard theory [76], in which a phase-field variable $\phi$ is defined such that the concentrations of the two phases in the interfacial 
region are $(1 \pm \phi) / 2$ and the functional for the free energy of the "mixing" phase is trucated at a square-gradient dependence $|\nabla \phi|^{2}$. However, this approach can only represent "weakly" nonlocal interactions, and does not properly capture the long-range intermolecular van der Waals attraction across a thin film [77].

The sharp interface approach treats interfaces between two macroscopic phases as mathematical surfaces of zero thickness and zero mass, across which the values of fluids' physical properties of the fluid such as viscosity and density jump discontinuously from one phase to another. This approach takes into consideration the local values of properties of interfacial tension and long-range interaction potentials to account for the differences in local molecular interactions. Navier-Stokes equations are solved along with appropriate boundary conditions to address the evolution of a multiphase system.

The computation of a sharp interface approach can be performed by interface capturing methods or interface tracking methods. The computation of an interface capturing method is based on a fixed spatial domain, where an interface function marking the interface position is computed. In interface tracking methods, the mesh needs to be updated as the flow evolves [78]. An interface capturing method is commonly implemented through an Eulerian reference frame, whereas interface tracking methods rely mostly on a Lagrangian representation of the coordinate system. Furthermore, both interface capturing methods and interface tracking methods can be derived based on stabilized formulations to prevent numerical oscillations and other instabilities with complex problems.

There are several methods to track an interface of a free surface problem discussed in the literature, which can represent the interface explicitly or implicitly and proceed with the tracking though Lagrangian or Eulerian approaches. The Front-Tracking method [79] and the Marker and Cell (MAC) method [80, 81] are examples of tracking strategies employed through a Lagrangian reference system and track and follow the interface according to the local velocity explicitly and implicitly, respectively. Both methods require a dynamic deletion or addition of particles when stretching or shrinking of the interface occur, and the MAC method requires a large number of particles to track the interface, which may scale the computational cost to solve such systems into unfeasible values.

Another important Lagrangian method for free surface analysis is the Smooth Particle Hydrodynamics (SPH) method, which relies on a meshless approach to numerically address the system dynamics. However, the use of artificial constants such as smoothing factors in the SPH method may induce spurious oscillations, in which additional steps may be required to obtain 
accurate solutions [82]. Particle finite element methods also adopt a purely Lagrangian framework, in which each node of the finite element mesh is tracked through time, along with element addition/removal strategies (Nguyen et al., 2008).

Nonetheless, the high computational cost of the tracking of large number of particles in a Lagrangian reference system led to the development of methods that use different strategies to capture the free surface position. The use of a marker (color) function in an Eulerian reference system instead of Lagrangian particles led to the development of interface capturing approaches such as the Level-Set (LS) method and the Volume of Fluid (VOF) method. The LS method (Osher and Sethian, 1988) identifies the interface through a smooth signed distance function $\gamma$, in which the interface lies in the zeroth level and its evolution is performed by advecting $\gamma$ at each time step. However, the magnitude of $\nabla \gamma$ can become too large or too small close to the interface, leading to inaccurate calculations of variables dependent of the interface position such as the normal vector and mean curvatures. Moreover, the method may also not conserve mas in flows with significant vorticity or with high deformation of the interface. To mitigate these problems, the distance function $\gamma$ must be reinitialized in every time step after the interface evolution process.

The VOF method [83] tracks the interface indirectly using a scalar marker $\alpha$ that denotes the volume fraction of the reference phase in each discrete element, taking $\alpha=1$ in regions fully occupied by the reference phase and $\alpha=0$ otherwise. The scalar marker function $\alpha$ is also known as the volume fraction function, and cells with $0 \leq \alpha \leq 1$ yields the interface position which is advected in time in an Eulerian manner. The VOF is one of the most widely used interface tracking methods and one of its advantages is that it presents good mass conservation properties due to its formulation. However, the accuracy on computation of curvature is still a key issue in the method, albeit several works aimed at overcoming the issue $[84,85]$. The VOF and LS methods track the interface implicitly and have similar ways to advect their respective marker function, and the main difference between the methods is that the LS marker function $\gamma$ is smooth, in contrast with the VOF step-like marker function $\alpha$. Moreover, both methods can lead to inaccurate curvature computations, which are known to generate spurious currents that result in non-physical pressure fields [86].

In short, Lagrangian descriptions allow an easy tracking of free surfaces and facilitates the treatment of materials with time-dependent constitutive relations but are unable to follow large distortions of the computational domain, whereas Eulerian descriptions can handle large distortions in the 
continuum motion with relative ease, but generally at the expense of precise interface definition and resolution of flow details [87]. A straightforward approach to tackle excessive mesh deformations consists in computing a new mesh that covers the deformed domain and then project the results from the deformed mesh to the new mesh, in a procedure often referred as mesh re-generation or remeshing. However, problems undergoing very large and quick deformations require repeated mesh re-generation and can thus become expensive in terms of computational time [88].

The shortcomings of purely Lagrangian and Eulerian descriptions lead to the development of an alternative technique that combines the best features of both descriptions in a mixed manner. Such techniques are known as arbitrary Lagrangian-Eulerian descriptions, and were first introduced by Hirt et al. [89] using a finite difference method. A formulation of the method using the finite element method was later introduced by Hughes et al. [90] to solve free surface flows and by Donea et al. [87] for transient dynamic Fluid-Structure Interaction (FSI) problems. An Arbitrary Lagrangian-Eulerian (ALE) description is very appealing for free surface problems due to its freedom in moving the mesh and its capability for an automatic reasoning that conserves the regularity of the computational mesh [91].

The ALE reference frame is identified by its instantaneous position vector $\xi$, which is a priori arbitrary and consequently independent of the motion of the mesh grid points. The position vector $\xi$ is linked to the material variables indirectly through the material coordinates in the initial configuration of the computational mesh. Thus, the ALE description is taken as a mapping of the initial configuration of the computational mesh into the current configuration of the reference frame. Figure 2.4 (adapted from [92]) portrays a schematic of free surface evolution for the aforementioned reference frames, in which the dashed line represent the initial configuration of the moving boundary, whereas the continuous line isits updated position. The left sketch in Figure 2.4 represents the Lagrangian approach to convect the free surface, in which the surface particles move along the direction of the solid red arrows that represent the material velocity.
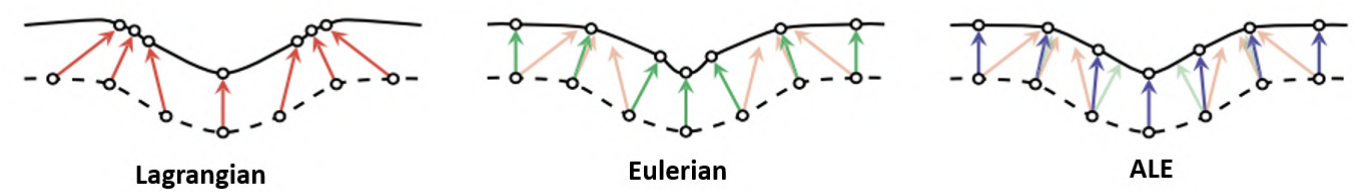

Figure 2.4: Comparative between Lagrangian, Eulerian and ALE methods to move the interface. 
The material velocity of the surface particles are represented in transparent red arrows on the middle and right sketches of Figure 2.4, concerning the Eulerian and ALE frames, respectively. The Eulerian approach moves the mesh on a previously defined, fixed mesh lines. In the example of Figure 2.4, this direction is taken to be orthogonal to the boundary, depicted as solid green arrows. The Eulerian reference frame provides regularity on the movement of surface particles in large in-plane flows, but may also squeeze the surface coordinates together in certain cases.

The ALE description treats the computational mesh as a reference frame which may be moving with an arbitrary velocity $\beta$. If $\beta=0$, the reference frame is fixed in space and corresponds to the Eulerian coordinate system, whereas $\beta=v$ indicates that the reference frame moves in space at the same velocity as the grid points, corresponding to the Lagrangian reference system. For the case $\beta \neq v \neq 0$, the reference system is called ALE frame and moves in space at a velocity $\beta$. The right sketch of Figure 2.4 shows the ALE method, where the surface points are arbitrarily displaced in the direction of the mesh velocity $\beta$, displaced as solid blue arrows. The mesh velocity $\beta$ is often linked to the normal component of the material velocity in the ALE method.

The choice of an appropriate description of the interface between fluid phases is therefore a fundamentally important consideration when developing numerical analyses of multiphase problems, as an ill-conditioned relation between the deforming continuum surface and its finite grid representation may yield unnacurate results or the inability to deal with large distortions. Tanaka and Kashiyama [93] introduced a robust ALE finite element method for FSI problems with a free surface using a mesh re-generation algorithm based on a background mesh that captures the position of the interfaces. The mesh re-generation is thus performed from the background mesh to the updated finite element mesh.

Later on, Ganesan and Tobiska [94] developed a coupled ALE-Lagrangian finite element method to compute the dynamics of insoluble surfactant on a free surface flow, in which the ALE method is used to track the surface and the surfactant concentration transport equation is approximated in a Lagrangian manner. You and Bathe [82] developed an improved numerical method that accurately describe transient solutions of 3D free surface flows using large time steps through the ALE method. The authors used the ALE method with a special focus on the condition of mass conservation during long-time response and introduce specific 3D elements for the free surface flow conditions evaluated therein.

Baiges et al. [88] presented an ALE method for numerical analyses of 
free surface flows using an adaptive finite element mesh covering a background domain that is capable of performing adaptative mesh refinement procedure on distributed memory environments. Stabilization terms were implemented to ensure a smooth solution for both velocity and pressure fields, which were in good agreement with experimental data. Then, Fumagalli et al. [95] used the ALE method to analyze the motion of a free surface in contact with a solid wall. In their study, surface tension, capillary effects and wall friction are accounted for both the determination of the contact angle between the free surface and the solid wall and the evolution of the surface dynamics.

Furthermore, Wang et al. [96] studied the bubble rising progress using a diffuse interface method to represent the interface and an ALE method to address the morphological changes of the bubble alongside an adaptative mesh refinement algorithm. More recently, Sahu et al. [92] developed an isoparametric ALE finite element method for incompressible fluid films with arbitrarily curved and deforming 2D interfaces that is independent of the inplane material flow. A finite element formulation to model the dynamics of lipid membranes with elastic behavior was also presented in the study.

In this work, we implement the ALE reference frame to track the motion of the interface. The details of the method are further discussed in Chapter 4 . 


\section{3}

\section{Problem formulation}

The problem analyzed here concerns the interplay between capillarity, interfacial rheology and long-range intermolecular van der Waals forces in the rupture of stationary free thin liquid sheets. Therefore, we aim at performing a numerical analysis of the problem dynamics towards the sheet rupture and analyze the effects of capillarity and interfacial viscosity on the rupture delay.

We employ a two-dimensional model of the problem and implement the One-Fluid model to numerically analyze the breakup dynamics of stationary free thin liquid sheet in a gaseous environment, considering the effects of surface tension, interfacial viscosity and van der Waals forces. The dynamics is evaluated under a symmetric long wave perturbation, concerning Taylor's findings. A symmetry plane on the $x$ axis is taken into account to ease the computational cost of the simulation. The modeling of the bulk hydrodynamics is done through the continuum approach, by means of conservation of mass and linear momentum along with proper boundary conditions.

The free surface is thought of as a sharp interface to account for the disjoining pressure in the system. The extra interface stress term to represent the viscous behavior of the interface is described using the Boussinesq-Scriven constitutive law. The system of differential equations is solved using the Finite Element Method and the free surface is tracked using the Arbitrary Lagrangian-Eulerian method. Moreover, we state that the surrounding gaseous phase is inert and therefore does not interact with the thin liquid sheet.

Figure 3.1 represents the initial configuration of the domain, in which $H_{C}$ is the undisturbed film thickness, $L_{C}$ is the perturbation wavelength and $\Gamma=1 \bigcup 3 \bigcup 2 \bigcup 3$ is the domain boundary. The free surface is marked with the $\operatorname{tag} 1$, the symmetry boundary is marked with 2 and left and right boundaries are marked with the tag 3 . The interface position is denoted as $h(x, t) \subset 1$, the initial conditions used are $u(x, y, t=0)=0$ and $h(x, t=0)=H_{C} / 2-\epsilon \cos (\pi x)$, where $\epsilon$ is the perturbation amplitude. The plane of minimum thickness is located at $x=0$. 


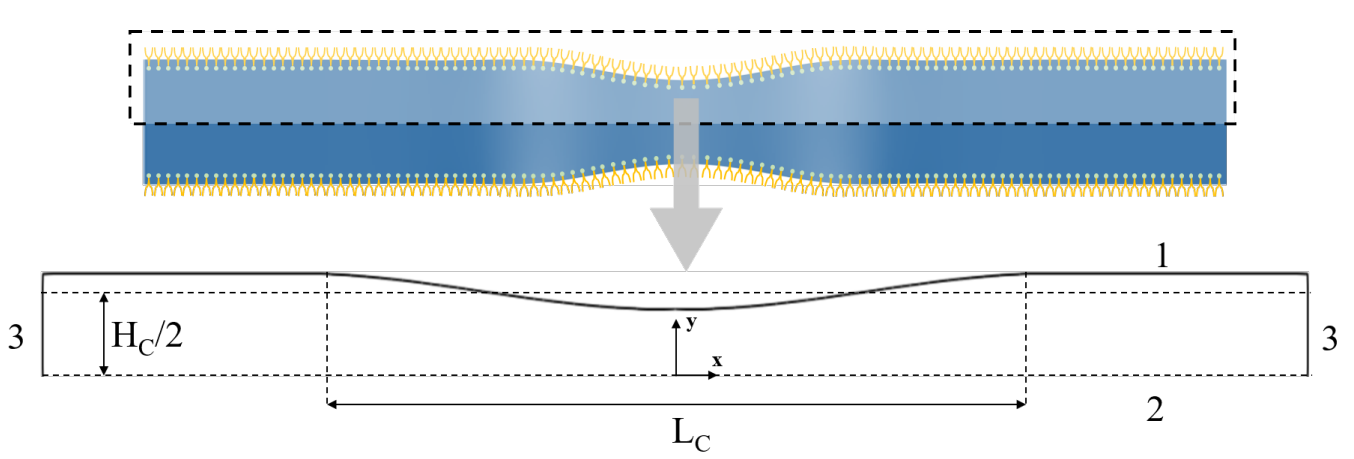

Figure 3.1: Initial configuration of the stationary free thin liquid sheet.

The numerical solution is based on the finite element method and is implemented in Python through the FEniCs open-source library. The simulation ends when the film minimum thickness achieves $h(x=0, t) \leq 0.1 H_{c}$.

\section{1}

\section{Governing equations}

\subsection{1}

\section{Bulk dynamics}

We consider the continuity equation (Eq. 3-1) for the conservation of mass and the Navier-Stokes equations (Eq. 3-2) for the conservation of linear momentum. Also, the fluid is considered incompressible and the system is isothermal. With these hypothesis, the conservation of mass is written as

$$
\nabla \cdot \mathbf{u}=0
$$

and the conservation of linear momentum is written as

$$
\rho\left(\frac{\partial \mathbf{u}}{\partial t}+\mathbf{u} \cdot \nabla \mathbf{u}\right)=\nabla \cdot T(\mathbf{u}, p)
$$

in which $T(\mathbf{u}, p)=-p I+2 \mu D(\mathbf{u})$ is the Cauchy stress tensor and $D(\mathbf{u})=$ $\left[\nabla \mathbf{u}+(\nabla \mathbf{u})^{T}\right]$ is the rate-of-deformation tensor. $\rho$ and $\mu$ are the bulk density and viscosity, respectively. 


\subsection{2}

\section{Interface dynamics}

Marangoni advection and thermal effects are neglected in our study, thus the term $\sigma\left(\Gamma_{c}, T\right)$ from the interfacial stress tensor $\sigma_{s}$ presented in Eq. 2-1 is a constant in our model.

$$
\sigma_{s}=\sigma_{\alpha \beta} I_{s}+\sigma_{e}
$$

The viscous behavior of the interface is described by the BoussinesqScriven law presented in Eq. 2-2. However, the expression for the extra interfacial stress can be substantially simplified in a $2 \mathrm{D}$ flow, as the free surface is a one-dimensional subdomain. The surface velocity divergence is defined as $\nabla_{s} \cdot \mathbf{u}=\operatorname{tr}\left(D_{s}\right)$, in which $\operatorname{tr}\left(D_{s}\right)=I_{s}: D_{s}$ is the trace of the surface rate-ofdeformation tensor. To that extent, the deviatoric part of $D_{s}$ can be written as $D_{s}^{*}=D_{s}-1 / 2\left(\nabla_{s} \cdot \mathbf{u}\right) I_{s}$.

The surface gradient operator can be written as $\nabla_{s}=\hat{\mathbf{t}} d / d s$, in which $s$ is the interface arclength coordinate and $\hat{\mathbf{t}}$ is the unit vector tangent to the free surface. The previous simplification is taken due to the fact that the projection tensor $I_{s}=\hat{\mathbf{t}} \hat{\mathbf{t}}+\hat{\mathbf{k}} \hat{\mathbf{k}}$ is reduced to $I_{s}=\hat{\mathbf{t}} \hat{\mathbf{t}}$ as $\hat{\mathbf{k}}$ is a constant unit vector in the z-direction. At last, we simplify the surface divergence as $\nabla_{s} \cdot \mathbf{u}=d u_{t} / d s$ and the surface rate-of-deformation tensor as $D_{s}=d u_{t} / d s \hat{\mathbf{t}} \hat{\mathbf{t}}$, which yields that $D_{s}^{*}=1 / 2\left(d u_{t} / d s\right)(\hat{\mathbf{t}} \hat{\mathbf{t}}-\hat{\mathbf{k}} \hat{\mathbf{k}})$. The term $u_{t}=\hat{\mathbf{t}} \cdot u$ is the velocity tangent to the interface. The simplification of the Boussinesq-Scriven law for a $1 \mathrm{D}$ viscous interface is reduced to

$$
\sigma_{e}=\left[\left(\kappa_{s}+\mu_{s}\right) \frac{d u_{t}}{d s}\right] \hat{\mathbf{t}} \hat{\mathbf{t}}+\left[\left(\kappa_{s}-\mu_{s}\right) \frac{d u_{t}}{d s}\right] \hat{k} \hat{k}
$$

Thus, the total interfacial stress tensor $\sigma_{s}$ in $2 \mathrm{D}$ flows is a contribution of curvature-dependent stress term and the viscous extra stress term.

$$
\sigma_{s}=\sigma_{\alpha \beta} I_{S}+\left[\left(\kappa_{s}+\mu_{s}\right) \frac{d u_{t}}{d s}\right] \hat{\mathbf{t}} \hat{\mathbf{t}}+\left[\left(\kappa_{s}-\mu_{s}\right) \frac{d u_{t}}{d s}\right] \hat{\mathbf{k}} \hat{\mathbf{k}}
$$

The surface divergence of the interfacial stress is

$$
\nabla_{s} \cdot \sigma_{s}=\left(\frac{d \eta_{s}}{d s} \frac{d u_{t}}{d s}+\eta_{s} \frac{d^{2} u_{t}}{d s^{2}}\right) \hat{\mathbf{t}}+\left(\sigma_{\alpha \beta}+\eta_{s} \frac{d u_{t}}{d s}\right) \frac{d \hat{\mathbf{t}}}{d s}
$$


where $\eta_{s}=\kappa_{s}+\mu_{s}$ is the total interfacial viscosity. The simple interface is recovered by setting $\eta_{s}=0$, as the extra viscous stress $\sigma_{e}$ is reduced to zero.

\subsection{3}

\section{Boundary conditions}

The solution of the drainage flow of the thin liquid film must satisfy the additional constrains along the boundaries of the domain. A first boundary condition at the liquid interface 1, as referred in Figure 3.1, is to assume that there is no mass transfer across the interface

$$
\hat{\mathbf{n}} \cdot \frac{d \mathbf{x}}{d t}=\hat{\mathbf{n}} \cdot \mathbf{u}
$$

A second boundary condition is given from the conservation of linear momentum across the interface, which yields a traction jump. The stress balance across the free surface is imposed as follows

$$
\hat{\mathbf{n}} \cdot T(\mathbf{u}, p)=\nabla_{s} \cdot \sigma_{s}-\left(p_{g}+\Phi\right) I_{s}
$$

where $p_{g}$ is the pressure of the gaseous phase and $\Phi$ is the long-range intermolecular van der Waals potential. To account for the long-range intermolecular attraction, the potential $\Phi$ is given as

$$
\Phi=\frac{\tilde{A}}{2 \pi h^{3}}
$$

in which $\tilde{A}$ is the Hamaker constant and $h$ is the thickness of the liquid film. The disjoining pressure is often modelled by assuming equilibrium van der Waals attraction between two unbounded parallel interfaces, albeit the free interfaces generally not being flat $[55,9]$.

The bottom boundary 2, as in Figure 3.1, is a symmetry line, where there is no flux across it and the tangential stress is null. In terms of the velocity field, these conditions can be written as

$$
\begin{gathered}
\mathbf{u}_{y}=0 \\
\frac{\partial \mathbf{u}_{x}}{\partial y}=0 .
\end{gathered}
$$


Moreover, the fluid is considered free to flow across left and right boundaries 3, which is a fictitious boundary of the domain, as depicted in Figure 3.1. We consider a zero pressure gradient in the $y$ along the left and right boundaries, which means that the pressure along the boundaries is given by the pressure at the point of intersection between left/right boundary and the free surface $\left(x_{l r}, y_{l r}\right)$. Since the interfacial stress acts on the tangential direction of the interface, when the interface is flat the boundary condition is taken as the normal-directed van der Waals force acting on the surface point of each left and right boundaries, respectively. Thus, the boundary condition for the lateral boundaries 3 is given as

$$
\begin{gathered}
\frac{\partial \mathbf{u}}{\partial x}=0, \\
p_{l r}=\frac{\tilde{A}}{2 \pi y_{l r}^{3}} .
\end{gathered}
$$

\section{2}

\section{Dimensionless parameters}

The scales on the $x$ and $y$ directions of the domain are taken as the perturbation wavelength $L_{C}$ and the undisturbed film thickness $H_{C}$, respectively. We introduce dimensionless variables using the following scales for time, velocity and pressure as

$$
T=\frac{\rho L_{C}^{2}}{\mu}, \quad \mathbf{U}=\frac{\mu}{\rho L_{C}}, \quad P=\frac{\mu^{2}}{\rho H_{C}^{2}} .
$$

Thus, the dimensionless variables are defined as follows

$$
x^{*}=\frac{x}{L_{C}}, \quad y^{*}=\frac{y}{H_{C}}, \quad t^{*}=\frac{t}{T}, \quad \mathbf{u}^{*}=\frac{\mathbf{u}}{U}, \quad p^{*}=\frac{p}{P} .
$$


We redefine the capillary number $C a$ and the Boussinesq number $B o$ in terms of the scales used in this work. As mentioned in section 2, the capillary number $C a$ is defined as the ratio between bulk viscous forces and interface tension forces, whereas the Boussinesq number measures the ratio between interface viscous forces and bulk viscous forces.

$$
C a=\frac{\mu U}{\sigma_{\alpha \beta}}, \quad B o=\frac{\eta_{s}}{\mu H_{C}}
$$

The ratio $H_{C} / L_{C}$ indicates the slenderness of the domain in terms of the undisturbed film thickness $H_{C}$ and the perturbation wavelength $L_{C}$. Considering a perturbation of amplitude $\varepsilon$, A long wavelength yields $H_{C} / L_{C}<<1$, meaning that the curvature of the free surface is smooth. On the other hand, the surface curvature becomes more pronounced as $H_{C} / L_{C}$ approximates unity.

We take into consideration the linear stability criterion proposed by Erneux and Davis [38] to evaluate the stability of thin liquid films with simple interfaces in our work. The authors consider the balance between interface tension forces and van der Waals forces to predict the sign of the rate of perturbation near $t=0$. A positive sign of the rate of perturbation indicates that the perturbation grows and the system undergoes rupture. A negative sign of the rate of perturbation indicates a retraction of the perturbation and the thin film is expected to recover a homogeneous thickness.

The predictions of the linear stability criterion are obtained in terms of the ratio between capillary forces and van der Waals forces. To that extent, the dimensionless parameters $S$ and $A$ are introduced. The parameter $S$ is the ratio between capillary forces and bulk viscous forces and is taken as the inverse of the capillary number:

$$
S=\frac{1}{3} \frac{1}{C a} \frac{H_{C}}{L_{C}}=\frac{\rho \sigma_{\alpha \beta} H_{C}}{3 \mu^{2}}
$$

The parameter $A$ is defined as the ratio between van der Waals forces and bulk viscous forces as below.

$$
A=\frac{\rho L_{C} \tilde{A}}{6 \pi H_{C}^{3} \mu^{2}}
$$


The linear stability criterion takes into consideration a long wavelength perturbation such that $H_{C} / L_{C}<<1$. To that extent, a thin liquid film with a simple interface is stable when

$$
\frac{S}{A}>\frac{2}{\pi^{2}}
$$

in which $S / A=2 \sigma_{\alpha \beta} H_{C}^{4} / L_{C} \tilde{A}$. 


\section{Numerical implementation}

The numerical solution of the problem is obtained through the finite element method. The computational domain is discretized by an unstructured mesh composed of triangular elements, in which the top boundary represents the free surface, bottom boundary is a symmetry line and left and right boundaries are outflow sections.

We use the FEniCS platform to compute the numerical solution of the problem. FEniCS is a collaborative project for the development of innovative concepts and tools for automated scientific computing and consists of a collection of interoperable components [97]. The framework is available in Python and $\mathrm{C}++$ programming languages and aims at providing high performance computation of finite element code generation while relieving the programmer of time-consuming and error-prone tasks in the creation of specialized codes. The FEniCS module is also equipped with parallel computing tools that allows process-wise degree-of-freedom indexing, full support for problems with over $2^{32}$ degrees of freedom for 64-bit indices and parallel mesh refinement strategy. We use the Python language [98] to implement the algorithms required to solve the system in the FEniCS platform. We use the built-in functions of FEniCS for the implementation of the finite element formulation and mesh generation and update.

This section describes the formulation of the finite element problem and the strategy used to employ computation of the system in the FEniCS platform. We provide a brief introduction to the finite element method and the derivation of the variational problem. We suggest the read of Gresho and Sani [99] and Carvalho and Valério [100] for a thorough overview of the basis and fundamentals of the finite element method and also Alnaes et al. [97] and Daversin-Catty et al. [101] for a more detailed introduction to the employment of automated solutions in the FEniCs platform. 


\section{1}

\section{The finite element method}

The finite element method is a numerical technique to obtain an adequate approximate solution of continuous problems using a finite number of welldefined basis functions. Elements $e_{i}$ associated with a specified number of nodes and vertices are organized in a common coordinate system $(x, y)$ as an assembly of finite elements that represent a domain of interest. Furthermore, the assembly of elements describing the domain of interest is referred as a mesh.

In this thesis we decompose the domain into triangular unstructured elements and use P2-P1 element pairs, also referred as Lagrange or TaylorHood elements, depicted in Figure 4.1. These type of elements yield a stable finite element discretization and high-order accuracy of approximations for the solution of the Navier-Stokes equations.
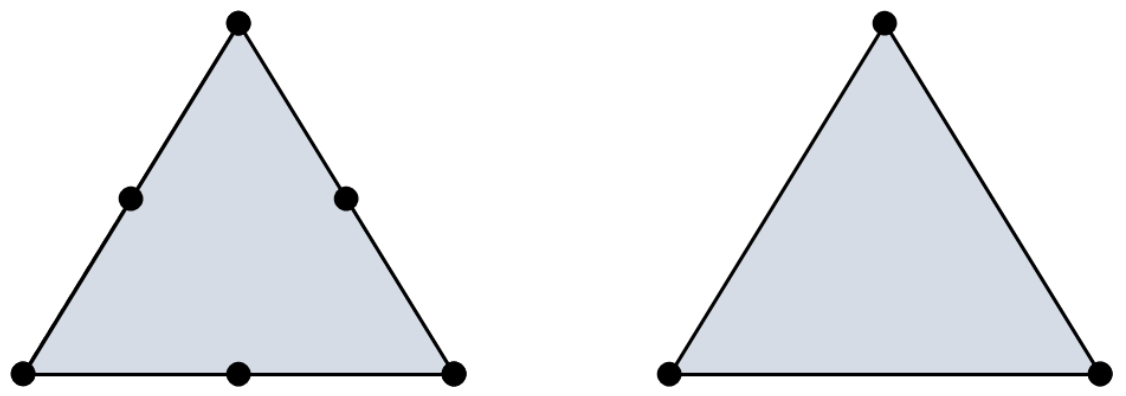

Figure 4.1: Quadratic (left) and linear (right) Taylor-Hood conforming elements.

For continuous problems described by a system of governing equations along with boundary and initial conditions, an accurate approximate solution can be achieved by a linear combination of appropriate basis functions $\psi_{i}(x)$. The approximated solution $u(x)$ is the combination of element-wise basis functions. The approximation $u(x)$ is written as

$$
u(x)=\sum_{i=1}^{N} c_{i} \psi_{i}(x)
$$

where $c_{0}, \ldots, c_{N}$ are unknown expansion coefficients to be determined in a way that approximates the solution of the system. We may also approximate the spatial derivatives of $u(x)$ by differentiating the basis function such that

$$
\frac{\partial u(x)}{\partial x}=\sum_{i=1}^{N} c_{i} \frac{\partial \psi_{i}(x)}{\partial x}
$$


An integral formulation of the system of partial differential equations is performed to ensure the obtention of $N$ linearly independent algebraic equations that approximate the system dynamics, in which $N$ is the number of discrete basis functions used to describe the approximate solution.

Thus, a set of algebraic equations is obtained by replacing $u(x)$ in the integral formulation of the original system of differential equations. The integral formulation of the problem is often performed by introducing weight functions $v_{i}(x) \in V$, where $V$ is a finite dimension function space that collects the weight functions.

The Weighted Residual method is often used to obtain an error measure for the approximate solution. It relies on defining a measure $R(x)$ such that $u(x)$ becomes the exact solution of the integral formulation of the problem if $R(x)=0$. The integration of the residual $R(x)$ along with the weight function $v(x)$ enforces that the weighted average of $R(x)$ is null, thus resulting in the weak form of the residual.

The Weighted Residual formulation is then rearranged in matrix form, which yields a matrix product in the form $\mathbf{A c}=\mathbf{b}$, in which $\mathbf{A}$ is sometimes called stiffness matrix, $\mathbf{b}$ is a vector of known values and $\mathbf{c}$ is the vector of expansion coefficients of the formulation. An approximated solution of the system dynamics is achieved by solving the aformentioned system to obtain the collection of expansion coefficients.

There are many techniques from which the approximations may be assumed, such as the point collocation method [102], subdomain collocation method [103] and the Galerkin method [104]. Our work is based on the Galerkin method, which takes $v_{i}=\Psi_{i}$.

The use of different kinds of finite element spaces for each function space is a normal approach among practitioners, wherein the selection of suitable function space properties may yield stable solution in multiphysics problems. Time-dependent probems require a time-stepping procedure that results in a system of partial differential equations at each time step. 


\section{2}

\section{Weak form and residual equation}

The weak form of a set of equations obtained by multiplying each differential equation by an appropriate arbitrary function and then integrate the product over the domain of the problem. Then, integration by parts may be used to reduce the order of derivatives to a minimum, followed by the introduction of boundary conditions if possible. Thus, considering $q(x) \in Q$ as an arbitrary weight function and $Q$ being a finite dimension scalar function space, the weak form of Eq. 3-1 is obtained as follows:

$$
\int_{\Omega}(\nabla \cdot \mathbf{u}) q d x=0
$$

The weak form of the Navier-Stokes equations is obtained analogously. We introduce an arbitrary weight function $v(x) \in V$, where $V$ denotes the space of vector functions, and multiply Eq. 3-2 by $v(x)$ and integrate over the domain. It goes as follows

$$
\int_{\Omega} \mathbf{v} \cdot\left[\rho\left(\frac{\partial \mathbf{u}}{\partial t}+\mathbf{u} \cdot \nabla \mathbf{u}\right)\right] d x=\int_{\Omega} \mathbf{v} \cdot[\nabla \cdot T(\mathbf{u}, p)] d x
$$

Integration by parts on the right-hand term reduces the order of derivatives

$$
\int_{\Omega} \mathbf{v} \cdot[\nabla \cdot T(\mathbf{u}, p)] d x=-\int_{\Omega} T(\mathbf{u}, p): \nabla \mathbf{v} d x+\int_{\Gamma}(\hat{\mathbf{n}} \cdot T(\mathbf{u}, p)) \cdot \mathbf{v} d s
$$

As mentioned in Figure 3.1, we split the boundary $\Gamma$ in the respective tags 1 for the free surface, 2 for the symmetry line and 3 for the outflow boundaries, so that the boundary conditions are introduced in the weak formulation. Hence, the surface stress condition presented in Eq. 3-8 in weak form is

$$
\int_{(1)}(\hat{\mathbf{n}} \cdot T(\mathbf{u}, p)) \mathbf{v} d s=-\int_{(1)} \hat{\mathbf{n}} \cdot\left(p_{s}+\Phi\right) \cdot \mathbf{v} d s+\int_{(1)}\left(\nabla_{s} \cdot \sigma_{s}\right) \cdot \mathbf{v} d s
$$


The term $\nabla_{s} \cdot \sigma_{s}$ is the second-order interface traction and requires an order reduction, since it contains a second derivative of the velocity along the interface. Therefore, we integrate the viscous contribution of the stress traction by parts. It goes as follows:

$$
\begin{array}{r}
\left.\int_{(1)}(\hat{\mathbf{n}} \cdot T(\mathbf{u}, p))\right) v d s=-\int_{(1)} \hat{\mathbf{n}} \cdot\left(p_{s}+\Phi\right) \cdot \mathbf{v} d s+\int_{(1)}\left(\sigma_{\alpha \beta} \kappa \hat{\mathbf{n}}\right) \cdot \mathbf{v} d s \\
+\eta_{s}\left[\int_{(1)}\left(\frac{d \mathbf{u} \cdot \hat{\mathbf{t}}}{d s} \frac{d \mathbf{v}}{d s}\right) \hat{\mathbf{t}} d s-\left.\frac{d \mathbf{u} \cdot \hat{\mathbf{t}}}{d s} \mathbf{v} \hat{\mathbf{t}}\right|_{s_{i}} ^{s_{f}}\right]
\end{array}
$$

in which the mean curvature of the free surface is $\kappa=-\nabla_{s} \cdot \hat{n} /\|\hat{n}\|$. We introduced $\eta_{s}=\kappa_{s}+\mu_{s}$ as a total interfacial viscosity, which is the only interfacial material property the model relies in $2 \mathrm{D}$ flows. Also, $s_{i}$ and $s_{f}$ denotes the extremities of the surface. Taking $\eta_{s}=0$ yields the behavior of a simple interface with no interfacial material properties. The kinematic condition presented in Eq. 3-7 is implemented by the ALE method and is discussed in the next section. Moreover, we naturally impose the boundary condition presented in Eq.3-13 for the boundaries marked with 3 as

$$
\int_{(3)}(\hat{\mathbf{n}} \cdot T(\mathbf{u}, p)) \cdot \mathbf{v} d s=-\int_{(3)} p_{l r} \hat{\mathbf{n}} \cdot \mathbf{v} d s
$$

As mentioned earlier, the boundary condition in Eq. 3-11 expresses the absence of shear stress on 2. This condition of a Neumann type, and is automatically satisfied if it remains homogeneous. The boundary condition presented in Eq. 3-10 enforces that there is no flow across the symmetry line. Thus, the $y$ component of the solution of the system is known and this implicates that the $y$ component of the weight function is zero. The weak form can be organized in terms of a residual $R$ as below.

$$
\begin{array}{r}
R=\int_{\Omega} \mathbf{v} \cdot\left[\rho\left(\frac{\partial \mathbf{u}}{\partial t}+\mathbf{u} \cdot \nabla \mathbf{u}\right)\right] d x+\int_{\Omega} T(\mathbf{u}, p): \nabla \mathbf{v} d x \\
+\int_{(1)} \hat{\mathbf{n}} \cdot\left(p_{s}+\Phi\right) \cdot \mathbf{v} d s-\int_{(1)}\left(\sigma_{\alpha \beta} \kappa \hat{n}\right) \cdot \mathbf{v} d s \\
-\eta_{s}\left[\int_{(1)}\left(\frac{d \mathbf{u} \cdot \hat{\mathbf{t}}}{d s} \frac{d v}{d s}\right) \hat{t} d s-\left.\frac{d \mathbf{u} \cdot \hat{\mathbf{t}}}{d s} \mathbf{v} \hat{\mathbf{t}}\right|_{s_{i}} ^{s_{f}}\right] \\
+\int_{(3)} p_{l r} \hat{\mathbf{n}} \cdot \mathbf{v} d s+\int_{\Omega}(\nabla \cdot \mathbf{u}) q d x \\
=0
\end{array}
$$

Furthermore, a time-marching scheme is required to forward the simula- 
tion in time. We consider the backward Euler method to evolve the system in time, with prescribed initial conditions. The backward Euler method is based on a truncated Taylor series expansion of an arbitrary solution in the neighborhood of $t=t^{n}$ as $y^{n}=y^{n-1}+\Delta t d y /\left.d t\right|_{t_{n}}+\mathcal{O}\left(\Delta t^{2}\right)$, in which the local truncation error is $\mathcal{O}\left(\Delta t^{2}\right)$ yields a first order method. Thus, provided an initial condition of the system, the term $\partial u / \partial t$ can be obtained as

$$
\frac{\partial u}{\partial t} \approx=\frac{u^{n}-u^{n-1}}{\Delta t}
$$

We consider an implicit time discretization and the solution of the nonlinear system of algebraic equations at each time step is done by the Newton-Raphson method. Considering the set $\left(u_{k}^{n}, p_{k}^{n}\right)$ as the solution of Eq. 4-7 at time $n$ and iteration $k$, the Newton-Raphson method is derived by representing $u_{k}^{n}$ as the sum of the initial guess $u_{k-1}^{n}$ and the correction term $\delta u$. We may rewrite the residual equation in terms of the iteration scheme $u_{k}^{n}=u_{k-1}^{n}+\delta u$ :

$$
\begin{array}{r}
R=\int_{\Omega} \mathbf{v} \cdot\left[\rho\left(\frac{\mathbf{u}_{k-1}^{n}+\delta u-\mathbf{u}^{n-1}}{\Delta t}+\left(\mathbf{u}_{k-1}^{n}+\delta u\right) \cdot \nabla\left(\mathbf{u}_{k-1}^{n}+\delta u\right)\right)\right] d x \\
+\int_{\Omega} T\left(\mathbf{u}_{k-1}^{n}+\delta u, p_{k}^{n}\right): \nabla \mathbf{v} d x \\
+\int_{(1)} \hat{\mathbf{n}} \cdot\left(p_{s}+\Phi\right) \cdot \mathbf{v} d s-\int_{(1)}\left(\sigma_{\alpha \beta} \kappa \hat{\mathbf{n}}\right) \cdot \mathbf{v} d s \\
-\eta_{s}\left[\int_{(1)}\left(\frac{d\left(\mathbf{u}_{k-1}^{n}+\delta u\right) \cdot \hat{\mathbf{t}}}{d s} \frac{d \mathbf{v}}{d s}\right) \hat{\mathbf{t}} d s-\left.\frac{d\left(\mathbf{u}_{k-1}^{n}+\delta u\right) \cdot \hat{t}}{d s} \mathbf{v} \hat{\mathbf{t}}\right|_{s_{i}} ^{s_{f}}\right] \\
+\int_{(3)} p_{l r} \hat{\mathbf{n}} \cdot \mathbf{v} d s+\int_{\Omega}\left(\nabla \cdot\left(\mathbf{u}_{k-1}^{n}+\delta u\right)\right) q d x \\
=0
\end{array}
$$

The algorithm for the Newton-Raphson method is obtained by rearranging Eq. 4-8 such that the left hand side of the equation accounts only for items evaluated at the $k$-th iteration. The Cauchy stress tensor in terms of the primitive variables is as below

$$
T\left(\mathbf{u}_{k-1}^{n}+\delta u, p_{k}^{n}\right)=-p_{k}^{n} I+2 \mu\left[\nabla \mathbf{u}_{k-1}^{n}+\left(\nabla \mathbf{u}_{k-1}^{n}\right)^{T}+\nabla \delta u+(\nabla \delta u)^{T}\right]
$$


Thus, the rearragement of Eq. $4-8$ yields

$$
\begin{array}{r}
\rho\left[\int_{\Omega} \frac{\delta u}{\Delta t} \cdot \mathbf{v} d x+\int_{\Omega}\left(\delta u \cdot \nabla \delta u+\mathbf{u}_{k-1}^{n} \cdot \nabla \delta u+\delta u \cdot \nabla \mathbf{u}_{k-1}^{n}\right) \cdot \mathbf{v} d x\right] \\
+\int_{\Omega} 2 \mu\left(\nabla(\delta u)+(\nabla(\delta u))^{T}\right): \nabla \mathbf{v} d x+\int_{\Omega}(\nabla \cdot \delta u) q d x-\int_{\Omega} p_{k}^{n} I \cdot \nabla \mathbf{v} d x \\
+\eta_{s}\left[\int_{(1)}\left(\frac{d(\delta u \cdot \hat{\mathbf{t}})}{d s} \frac{d \mathbf{v}}{d s}\right) \hat{\mathbf{t}} d s-\left.\frac{d(\delta u \cdot \hat{\mathbf{t}})}{d s} \mathbf{v} \hat{\mathbf{t}}\right|_{s_{i}} ^{s_{f}}\right] \\
\rho\left[\int_{\Omega} \frac{\mathbf{u}^{n-1}-\mathbf{u}_{k-1}^{n}}{\Delta t} \cdot \mathbf{v} d x-\int_{\Omega}\left(\mathbf{u}_{k-1}^{n} \cdot \nabla \mathbf{u}_{k-1}^{n}\right) \cdot \mathbf{v} d x\right]-\int_{\Omega}\left(\nabla \cdot \mathbf{u}_{k-1}^{n}\right) q d x \\
-\int_{(1)} \hat{\mathbf{n}} \cdot\left(p_{s}+\Phi\right) \cdot \mathbf{v} d s+\int_{(1)}\left(\sigma_{\alpha \beta} \kappa \hat{\mathbf{n}}\right) \cdot \mathbf{v} d s-\int_{(3)} p_{l r} \hat{\mathbf{n}} \cdot \mathbf{v} d s \\
+\eta_{s}\left[\int_{(1)}\left(\frac{d\left(\mathbf{u}_{k-1}^{n} \cdot \hat{\mathbf{t}}\right)}{d s} \frac{d \mathbf{v}}{d s}\right) \hat{\mathbf{t}} d s-\left.\frac{d\left(\mathbf{u}_{k-1}^{n} \cdot \hat{\mathbf{t}}\right)}{d s} \mathbf{v} \hat{\mathbf{t}}\right|_{s_{i}} ^{s_{f}}\right]
\end{array}
$$

The equation above may be rewritten in matrix form as $K \delta u=b$, in which $K$ is often referred as the stiffness matrix and $b$ is a vector composed of known terms evaluated at iteration $k-1$. Analogously, Eq. 4-8 can be linearized in terms of a correction term $\delta u$, resulting in $R(u)=R\left(u_{k-1}+\delta u\right)=0$. This procedure yields

$$
R\left(\mathbf{u}_{k-1}\right)+\left.\frac{\partial R}{\partial \mathbf{u}_{k-1}}\right|_{\mathbf{u}_{k-1}} \delta u=0
$$

Rearranging the equation above leads to

$$
\left.\frac{\partial R}{\partial \mathbf{u}_{k-1}}\right|_{\mathbf{u}_{k-1}} \delta u=-R\left(\mathbf{u}_{k-1}\right) .
$$

The expressions portrayed by Eq. 4-9 and Eq. 4-10 are equivalent. The selection of appropriate finite function spaces $U$ for the basis functions and $V$ and $Q$ for the weight functions is required for the assembly of the system in matrix form and solution of the system for time $t_{n}$. Then, the solution of the system is obtained by solving Eq. 4-9 for the correction term $\delta u$ and a updating $u_{k}^{n}$ iteratively until a set of criteria is achieved. This leads to the algorithm below:

The definition of the convergence criteria and iteration tolerance is further discussed in Section 4.4 along with the implementation of the system 


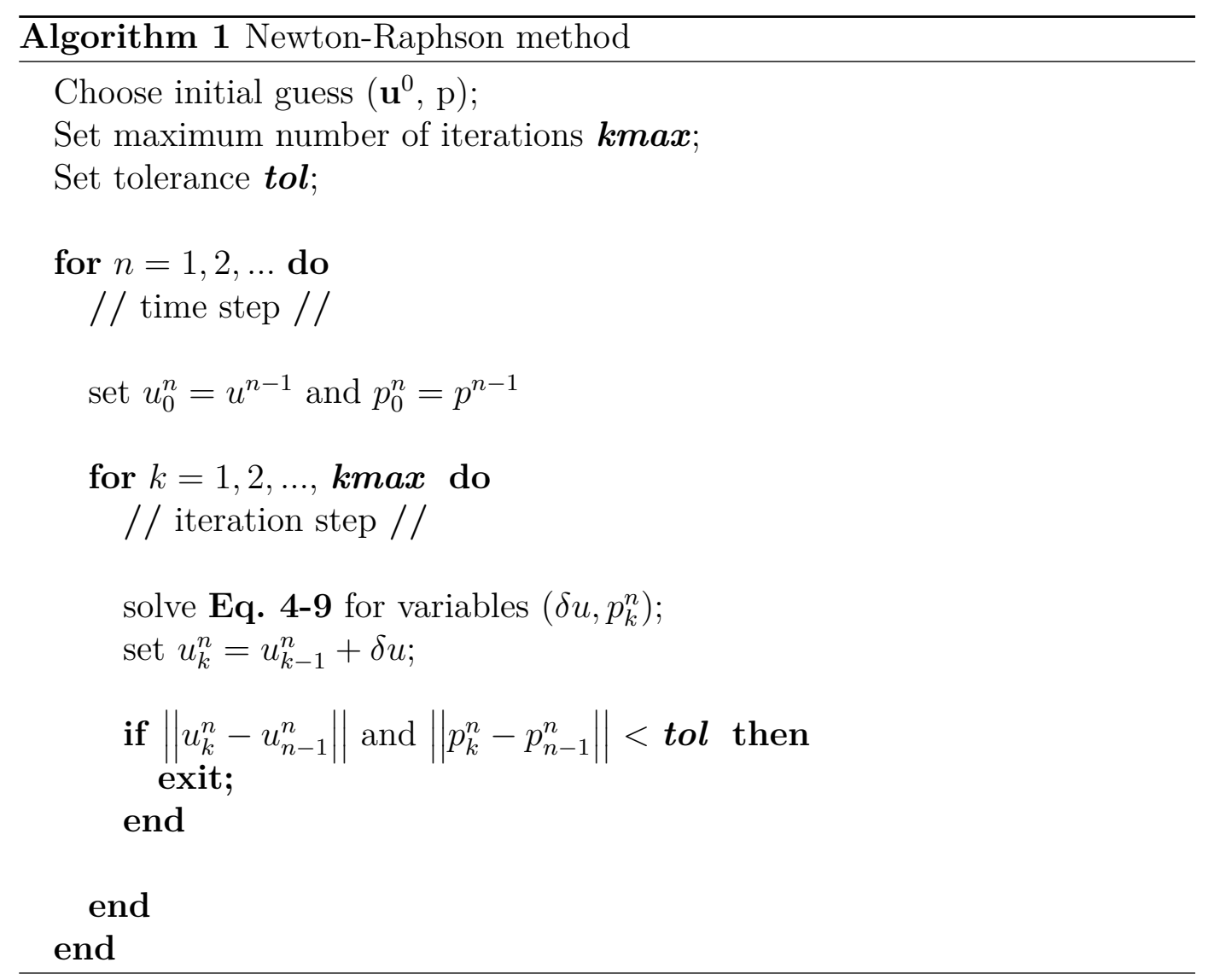

in FEniCS.

\section{3}

\section{Arbitrary Lagrangian-Eulerian method}

As previously mentioned, an arbitrary Lagrangian-Eulerian approach has features of both Lagrangian and Eulerian methods and allows independent movement of the grid points in a time increment $\Delta t$ with a grid velocity $\beta$. To that extent, the ALE description of movement aims at providing a generalized description capable of combining at best the interesting aspects of the material and spatial descriptions while minimizing their drawbacks as far as possible [105].

The Lagrangian viewpoint consist on a material domain $\mathcal{R}_{\boldsymbol{X}}$ composed of material particles $X$, whereas the Eulerian description yields a spatial domain $\mathcal{R}_{x}$ composed by a set of spatial points $\boldsymbol{x}$. The mapping between Lagrangian and Eulerian reference frames is such that the spatial coordinates $\boldsymbol{x}$ depend on the material particle $\boldsymbol{X}$ and the physical time measured by the same variable $t$ in both material and space reference frames.

As the material points coincide with the grid points during the motion, there are no convective effects in this frame of reference. In an Eulerian reference frame, the finite element mesh is fixed and the continuum moves and 
deforms with respect to the computational grid. Thus, an Eulerial description of motion involves only variables and functions having an instantaneous significance in a fixed region of space. In this case, the material velocity $v$ corresponds to the velocity of the material point and is expressed with respect to the fixed-element mesh without any reference to the initial configuration of the continuum.

The dissociation of the mesh nodes from the mesh particles lead to the occurrence of convective effects, which are due to the relative motion between the deforming material and the fixed mesh. The ALE method takes place in a referential configuration $\mathcal{R}_{\xi}$, where $\xi$ is the reference coordinate that identifies the grid points. Concerning Donea et al. [105], the Lagrangian and Eulerian formulations may be obtained as particular cases according to an appropriate choice of mappings between the reference systems.

The most challenging problem in an ALE algorithm is allocating an appropriate value for $\beta$, as there is no general way to determine the mesh velocity. A practical implementation of the ALE description can be overshadowed by the burden of specifying grid velocities well suited to the particular problem under consideration and most likely requires that an automatic mesh displacement prescription algorithm is supplied.

The update of the mesh in the ALE formulation is performed mainly by two basic strategies, namely mesh regularization and mesh adaptation. Mesh regularization is often referred as rezoning, and it consists in keeping the computational mesh as regular as possible during the whole calculation, thus avoiding excessive distortions and squeezing while maintaining an accurate representation of the physical problem. This approach requires that the nodal coordinates are updated at each iteration, either through step displacements or from the computed velocities of the problem.

There are several algorithms to regularize the mesh, such as the transfinite mapping method [106], the Laplacian smoothing [107] and the simple interpolation scheme $[87,108]$. These algoritmhs interpolate the motion of the mesh throughout the domain according to a specific procedure, such as in the Laplace smoothing procedure, where the Laplace equation is solved for each component of the node velocity or position so that on a logically regular region the mesh forms lines of equal potential. In fact, the ALE method allows the use of any mesh-smoothing algorithm designed to improve the shape of the elements once the topology is fixed.

In the mesh adaptation scheme, the objective of the ALE description is to optimize the computational mesh to achieve an improved accuracy at possibly low computing cost, as the number of mesh elements may remain 
unchanged througout the computation. Thus, the ALE method includes an indicator of error and the mesh is modified to obtain an equi-distribution of the error over the entire computational mesh. The ALE method can also be coupled with traditional mesh refinement procedures such as $h$-adaptivity to further enhance accuracy of the computation [109].

In this work, the ALE method tracks the motion of the free surface by employing the kinematic boundary condition presented in Eq. 3-7 to update the position of the free boundary over the computation. More specifically, we use the normal component of the velocity field to update free surface position at each interation. Then, the algorithm performs a mesh regularization procedure on the bulk elements. The update of the surface motion is performed by the built-in packages of the FEniCs library, which by default rezones the computational domain by the Laplacian smoothing technique.

The Laplacian smoothing technique relies on the solution of the Laplace equation for each coordinate direction to compute new coordinates for all vertices, provided new boundary coordinates. Therefore, our method to displace the boundary updates the coordinates of the free surfaces with the ALE method according to the normal velocity of the boundary and then solves the the equation $\nabla^{2} \delta_{s}=0$ to update the coordinates of the bulk vertices with the displacement vector $\delta_{s}$.

\section{4 \\ Numerical procedure}

The procedure to obtain the numerical solution of the problem concerns mesh creation, finite element formulation and the solution of the system. Then, we solve the system using an iterative method to obtain a numerical solution associated with a predefined set of liquid properties and flow conditions.

The mesh generation functionality of FEniCS is accounted by the mshr function, which allows simple generation of meshes from a combination of Constructive Solid Geometry descriptions and Boolean operations. The creation of the computational mesh is a straightforward operation performed by the $\mathrm{mshr}$ module. The boundaries of the mesh are marked with a respective tag for each boundary and are stored in an auxiliary function. Figure 4.2 shows a coarse computational mesh of triangular finite elements created by the mshr library.

Figure 4.2: Computational mesh obtained using the $m s h r$ package. 
The assembly of linear and nonlinear systems in the FEniCS is automated by the DOLFIN module. As previously mentioned, we adopt Lagrange elements, which are unstructured triangular finite elements composed of a P2P1 element pair, namely a pair of quadratic and linear polynomial basis functions, respectively. Then, we take the Galerkin method into consideration to define the appropriate function spaces.

Once the mesh and the function spaces are defined, we declare the material properties of the system, such as bulk viscosity and density, interface viscosity, interfacial tension and Hamaker constant. Afterwards, we employ the Newton-Raphson method to solve Eq. 4-9 iteratively at each time step. The absolute and relative tolerances used in the iterative procedure is $10^{-14}$ and $10^{-16}$, respectively.

The velocity field obtained in the iterative procedure is then used to update the free surface movement, by means of the kinematic condition presented by Eq. 3-7. The ALE method then moves the nodes of the free boundary into a new position in a Lagrangian manner, meaning that the free boundary displacement is obtained directly from $x_{s}=u_{s} \Delta t$. After, the computational mesh is reorganized according to the Laplacian smoothing technique.

We initiate the simulation with $\Delta t=10^{-7} \mathrm{~s}$ and employ an adaptative time step scheme inspired by the Courant-Friedrichs-Lewy (CFL) condition. The CFL condition states that the distance in which physical information travels during one time step within the mesh must be lower than the distance between mesh elements [110]. It is a necessary condition for convergence while solving certain partial differential equations with explicit time integration schemes [111]. The use of an implicit solver usually alleviates numerical instabilities that arise from the CFL condition. Thus, we define an artificial Courant number $\overline{C o}=\left\|u_{m} a x\right\| \Delta t / \Delta x$ to update of the time step as

$$
\Delta t=\frac{\overline{C_{o}} \Delta x}{\left\|\mathbf{u}_{\max }\right\|}
$$

Therefore, the time step decreases as the magnitude of the maximum velocity increases for a given mesh resolution $\Delta x$. We enforce the aforementioned relation between the velocity and time step mainly because as the thin liquid sheet evolves towards rupture, van der Waals forces become more dominant and the thinning of the film accelerates. More briefly, we increase the time resolution near the rupture of the thin liquid sheet by maintaining a fixed value for $\overline{C o}$ in the update procedure. 
Furthermore, Algorithm 2 shows the solution procedure implemented in this work. The results discussed in the next section are saved in general formats such as .pvd and .npy to ease the post-processing task. Moreover, the postprocess of the results obtained from the solution procedure is performed in Paraview and Python.

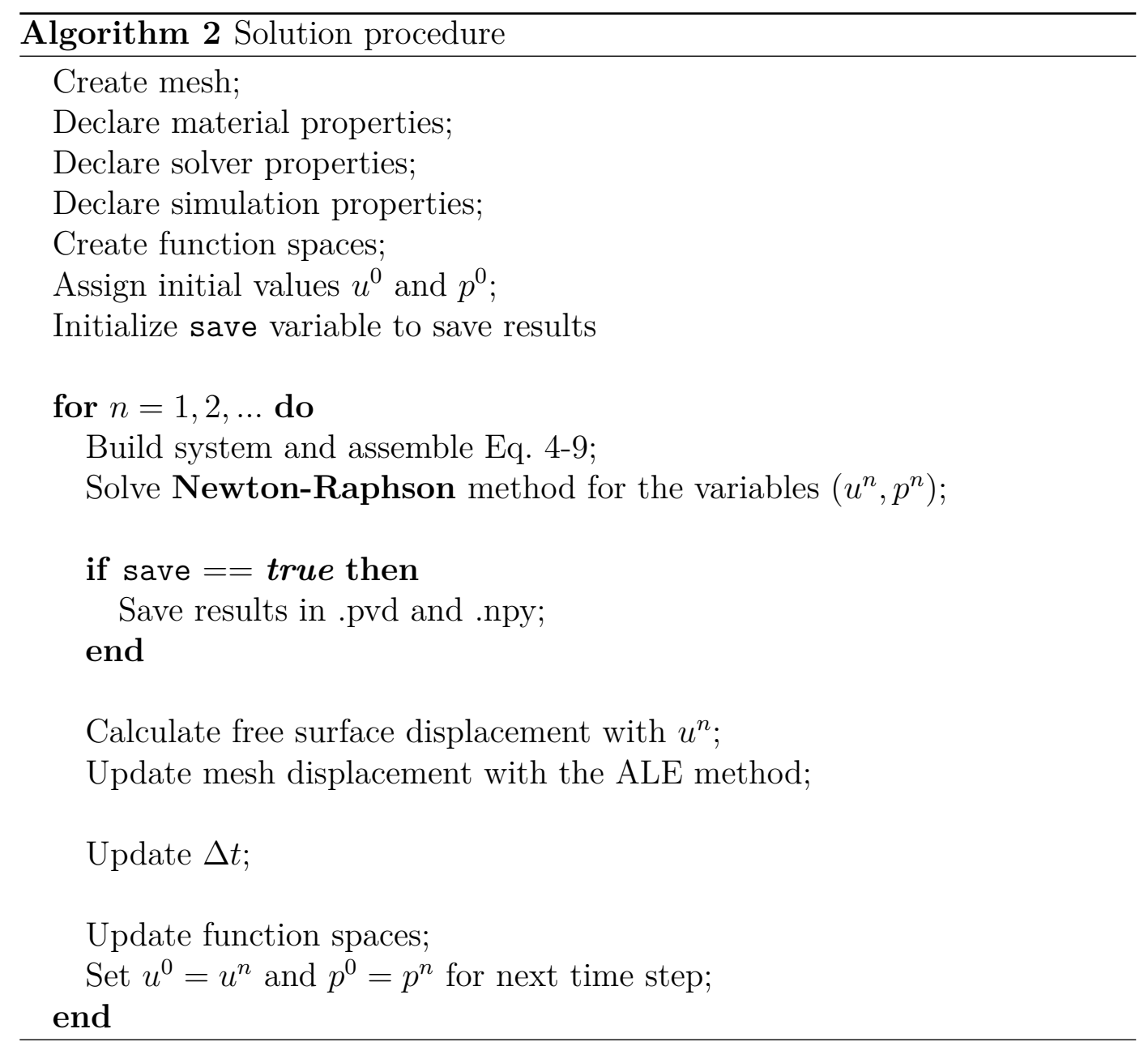




\section{5}

\section{Results and discussion}

In this chapter, we present and discuss the results obtained for the rupture dynamics of thin free liquid sheets. The dynamics of the system is numerically addressed using the system of equations in dimensional form, considering an aqueous solution for reference values of bulk density and viscosity and null initial conditions for the velocity and pressure. Moreover, the domain is configured for $H_{C}=10 \mu \mathrm{m}$ and $L_{C}=10 H_{C}$, and the results presented in this chapter are scaled according to the dimensionless variables presented in Section 3.2.

We take into consideration the stability criterion of free thin liquid sheets introduced in Section 3.2 to account for the balance between capillarity and intermolecular attraction. The numerical solutions discussed in this chapter is obtained by fixing the capillary number at $C a=2$. The analysis of the dynamics of thin liquid films with viscous interfaces is performed for values of the Boussinesq number in the range $B o \in[0,75]$. As previously mentioned, the simulations end when the minimum thickness of the liquid sheet reaches the critical value $h_{f}=0.2 H_{C}$, which is the criterion considered in this work to address the rupture event that occurs in a finite time.

The simple case taken at $C a=2$ and $B o=0$ is used to assess convergence issues regarding the mesh resolution, which is further discussed in Section 5.2. We evaluate the results obtained for the rupture dynamics of thin liquid sheets with a simple interface $(B o=0)$ in Section 5.2. Then, we introduce the results that concern to the effects of interfacial viscosity in the rupture dynamics of the thin film in Section 5.3.

\section{1}

\section{Mesh convergence test}

We analyze the influence of the mesh resolution on the convergence of the numerical method by comparing the time that each simulation reaches the minimum thickness criterion of $h_{f}=0.2 H_{C}$. The parameters are such that $S / A=1 / \pi^{2}$ and an initial perturbation of $\epsilon=0.1 H_{C}$ is imposed. At this value of $S / A$, the flow is unstable and the rupture of the free liquid film occurs. We test if the simulation converges for a given number of elements that compose the computational domain, according to an appropriate time step.

The update of the time step is obtained by varying the artificial Courant number $\overline{C o}$ between 100 and 1000, as defined in Section 4.4. The results 
obtained for the range $\overline{C o} \in[100,1000]$ indicate similar rupture times for a fixed mesh resolution. Thus, we kept $\overline{C o}=500$ fixed for the results presented herein.

The thickness evolution at $x=0$ for different mesh resolution is presented in Figure 5.1. The computational domain is discretized with $\mathrm{N}$ number of elements such that $N \in[4500,12000,32000,37000,44000]$ to account for both coarse and refined grids.

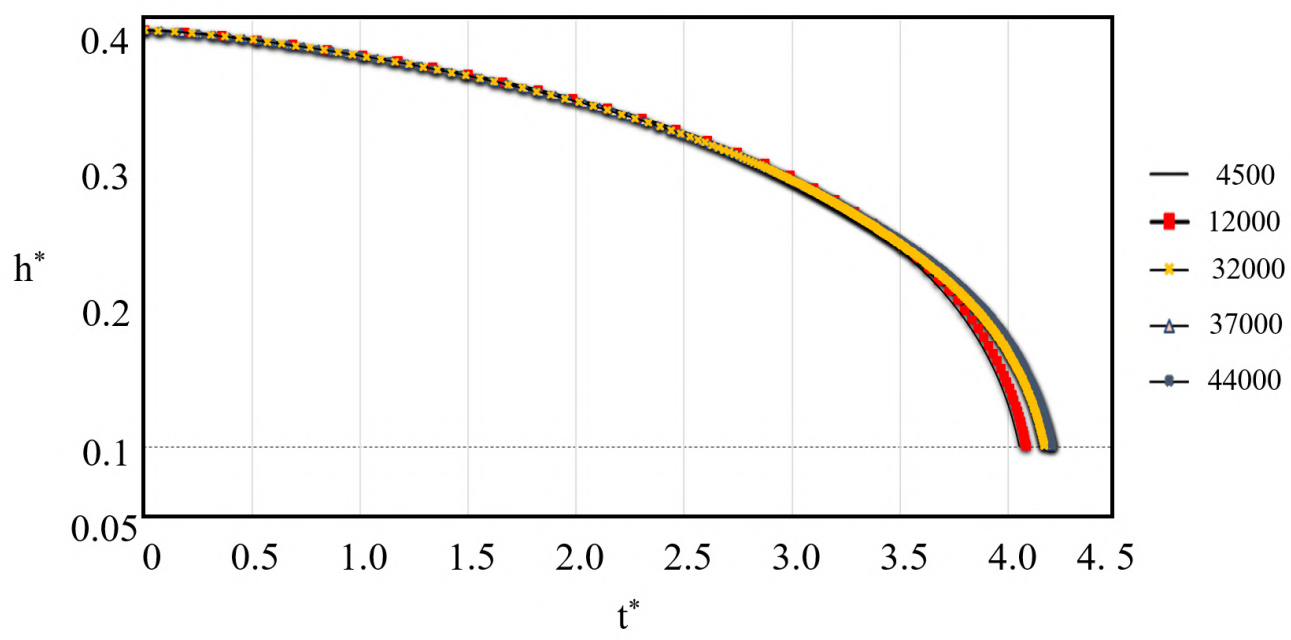

Figure 5.1: Breakup dynamics for different mesh resolutions.

Figure 5.2 depicts a close-up view of the rupture times for each discretization. These results indicate that the simulations converge for rupture times of the same order of magnitude, regardless of the coarse and refined meshes analyzed. However, as we increase the mesh resolution, the breakup time converges to $\mathrm{t}_{f}^{*} \approx 4.3$.

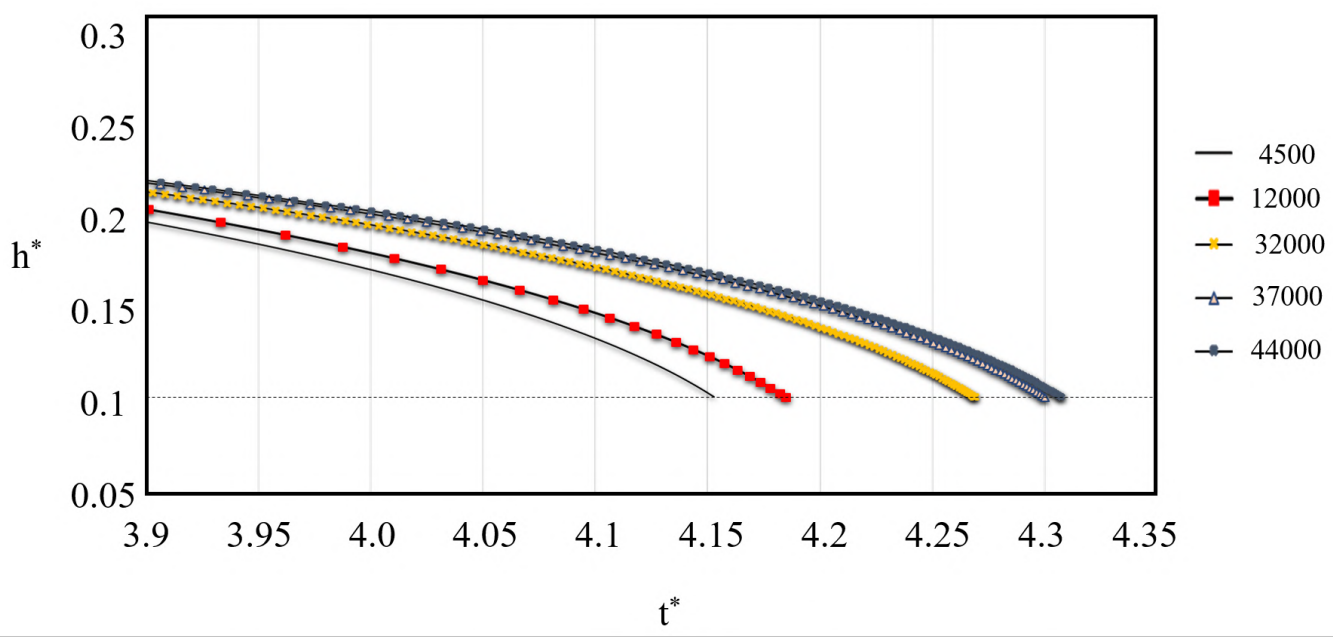

Figure 5.2: Closer look on the thickness profile at rupture neighbouring. 
Furthermore, the trade-off between a highly refined domain and the time of computation associated with the system must be addressed. Therefore, we consider a mesh with $N=37000$ in our study, which yields a computation time of $T \approx 12 h$ for the simulation of the results presented hereby.

\section{2}

\section{Simple interface}

Capillary effects due to the surface curvature introduce a pressure gradient acting on the tangential direction of the free surface, which drives the liquid towards the minimum thickness plane. Long-range intermolecular van der Waals forces act on the normal direction of the free surface, yielding an attraction between the free surfaces of the film and leading the sheet to rupture. Hence, capillary effects are expected to oppose the long range intermolecular attraction between the surfaces of the free thin liquid film. For $S / A \geq 2 / \pi^{2}$, the capillary forces are strong enough to stabilize the flow.

The results of the simple interface case are presented in this section. We investigate the behavior of stationary thin liquid films for $1 / \pi^{2} \leq S / A \leq 3 / \pi^{2}$. The ratio $S / A$ provides insights about the behavior of the system under certain flow conditions, in which the critical value of $S / A=2 / \pi^{2}$ is a bifurcation point. According to the linear stability criterion, the system is stable and the film does not rupture for $S / A>2 / \pi^{2}$, whereas the system is unstable for $S / A \leq 2 / \pi^{2}$ and the film undergoes rupture.

As defined in Chapter 4, an artificial Courant number is used to obtain an adaptive time step for the temporal evolution of the system. We apply $\overline{C o}=500$ for the cases of $S / A<2 / \pi^{2}$, as the rupture dynamics under this configuration speeds up as we decrease the stability ratio $S / A$. Analogously, we implement $\overline{C o}=1000$ to march in time with larger time steps for $S / A \geq 2 / \pi^{2}$.

\subsection{1}

\section{Literature comparison}

We validate our numerical implementation by comparing our results with the predictions obtained by the linear stability criterion proposed by Erneux and Davis [38]. To that extent, we analyze the temporal evolution of the film thickness. Therefore, concerning the symmetry of our domain, we introduce the following variable to represent the dynamics of thin sheets under perturbations:

$$
\omega(t)=\frac{H_{C}}{2}-h(x=0, t) .
$$


The investigation of the stability criterion proceeds by considering an initial perturbation of amplitude $\epsilon_{0}=0.02 H_{C}$, yielding $\omega_{0}=0.02 H_{C}$. Then, we change the values of $S / A$ and evaluate the accuracy of the linear stability analysis in predicting the behavior of the dynamical system. Furthermore, we may evaluate the temporal evolution of the perturbation with respect to the initial perturbation as:

$$
\varepsilon(t)=\omega(t)-\omega_{0}
$$

$\varepsilon(t)>0$ indicates that the perturbation grows with time and that the liquid sheet is unstable. On the other hand, negative values of $\varepsilon(t)$ indicate that the perturbation is damped and the sheet is stable. Linear stability analysis predicts that the liquid sheet is unstable for $S / A$ less than the critical value of $S / A=2 / \pi^{2}$. Figure 5.3 presents the evolution of the perturbation $\varepsilon(t)$ for $1 / \pi^{2}<S / A<3 / \pi^{2}$.

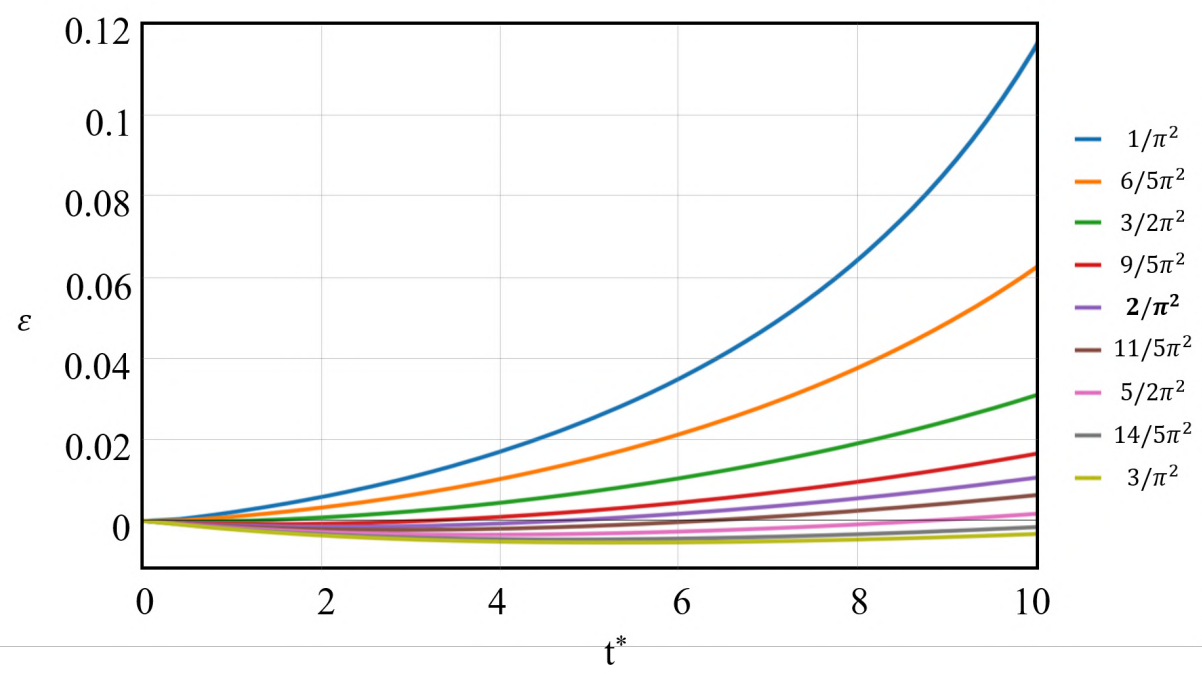

Figure 5.3: Perturbation evolution in time for $1 / \pi^{2} \leq S / A \leq 3 / \pi^{2}$.

Figure 5.4 presents a zoom of the perturbation evolution near $t=0$, which is related to linear stability response, valid at small perturbations. 


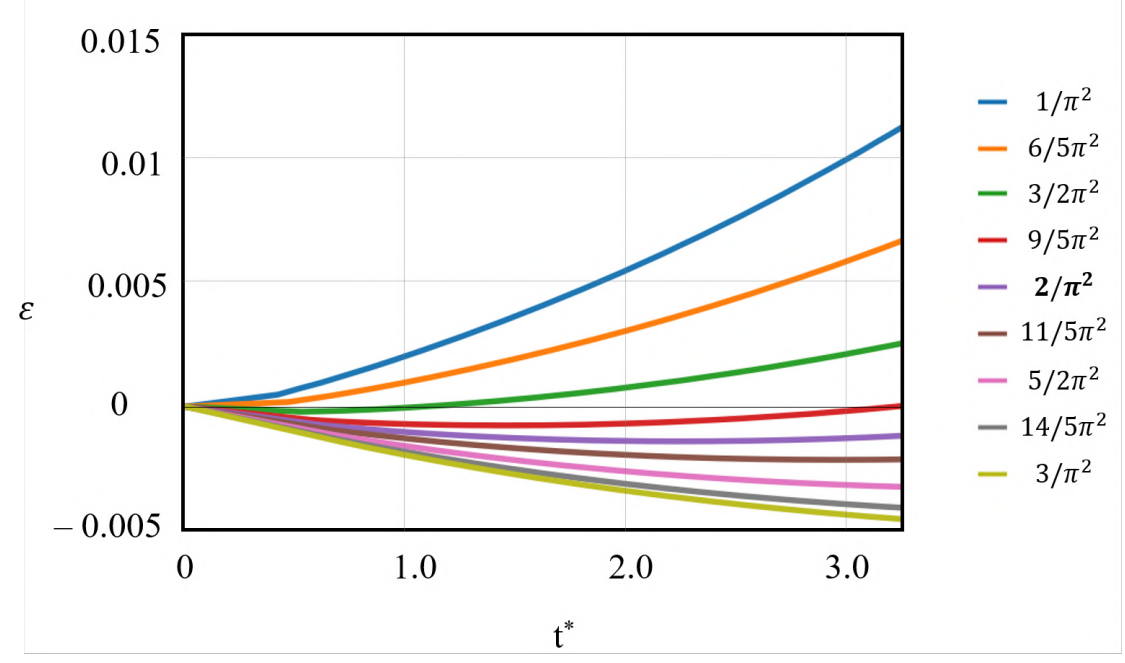

Figure 5.4: Close-up on the early stages of the perturbation evolution for $1 / \pi^{2} \leq S / A \leq 3 / \pi^{2}$.

At small values of $S / A$, i.e. $S / A \leq 6 / 5 \pi^{2}, \varepsilon(t)>0$ for all values of $t$. The perturbation grows until the sheet breakup. At intermediate values of $S / A$, i.e. $3 / 2 \pi^{2} \leq S / A \leq 11 / 5 \pi^{2}$, the perturbation presents a non-monotonic behavior, it first decays but then it starts to grow after some time. At large values of $S / A$, i.e. $S / A>5 / 2 \pi^{2}$, the perturbation always decay $(\varepsilon(t)<0$ for all $t)$ and a film with uniform thickness is recovered.

It is important to note that the linear stability analysis cannot predict this non-monotonic behavior. It only determines the sign of the growth rate at vanishingly small perturbations, close to $t=0$. The non-monotonic behavior is associated with nonlinear effects neglected by the linear stability analysis.

From the predictions of the sign of $d \varepsilon / d t(t \rightarrow 0)$ from the nonlinear flow solution, which can be determined from Figure 5.4, the sheet is stable for $S / A>3 / 2 \pi^{2}$. Figure 5.5 depicts a comparison between our results concerning the perturbation $\varepsilon$ at $t^{*}=1$ and similar predictions of Bazzi and Carvalho [9]. 


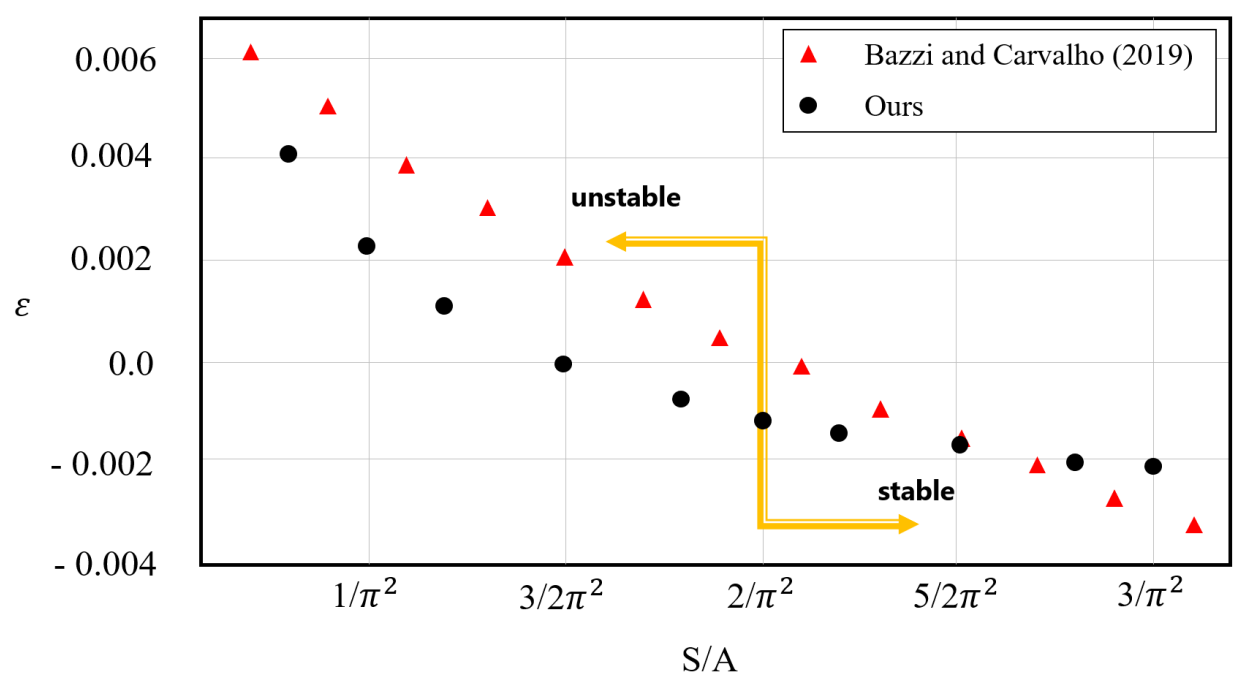

Figure 5.5: Comparison of the results obtained perturbation amplitude at $t^{*}=1$ with the literature.

These results also indicate that the perturbation retracts for $S / A>3 / 2 \pi^{2}$ and grows otherwise. We also notice that the way at which the perturbation growth varies with $S / A$ differs from the results presented by Bazzi and Carvalho [9]. This behavior may be due to the differences in our modeling. They used lubrication theory to derive a set of nonlinear equations, whereas our model solves the complete Navier-Stokes equations at which inertial forces are not neglected.

We present the perturbation $\varepsilon$ as function of $S / A$ at $t^{*}=2.1$ and $t^{*}=4$ in Figure 5.6. According to our results presented in Figures 5.3 and 5.4, the growth of the perturbation for $S / A=2 / \pi^{2}$ stops retracting at $t^{*}=2.1$. The perturbation amplitude at $t^{*}=4$ indicates that system evolves towards rupture. 


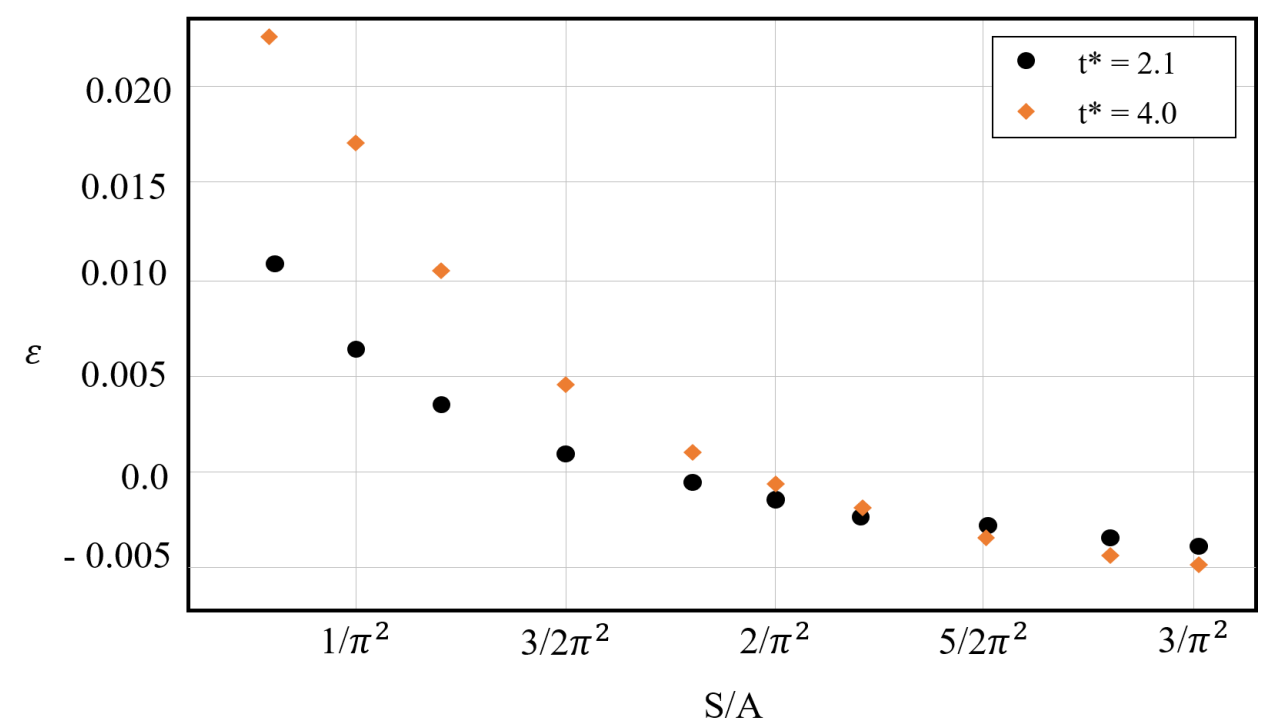

Figure 5.6: Perturbation growth for $1 / \pi^{2} \leq S / A \leq 3 / \pi^{2}$ at $t^{*}=2.1$ and $t^{*}=4$.

Thus, the critical stability ratio $S / A=2 / \pi^{2}$ results in a retraction of the perturbation up until $t^{*}=2.1$, and then the liquid sheet undergoes a finite time rupture. This behavior is also observed for $2 / \pi^{2} \leq S / A \leq 3 / \pi^{2}$, where the balance between capillarity and intermolecular van der Waals forces are no longer accurately predicted by the stability criterion after a given time.

We remark that the predictions proposed by the the stability criterion are obtained by neglecting nonlinear effects on the system dynamics, which may lead to innacurate forecasts. Moreover, the evolution of the liquid film thickness under a perturbation amplitude of $\epsilon=0.1 H_{C}$ and stability ratios of $S / A=1 / \pi^{2}$ and $S / A=3 / \pi^{2}$ is depicted in Figure 5.7. According to the findings of Bazzi and Carvalho [9], the aforementioned configuration should still yield a retraction of the perturbation for $S / A=3 / \pi^{2}$ and a perturbation growth for $S / A=1 / \pi^{2}$, albeit the larger initial perturbation. 


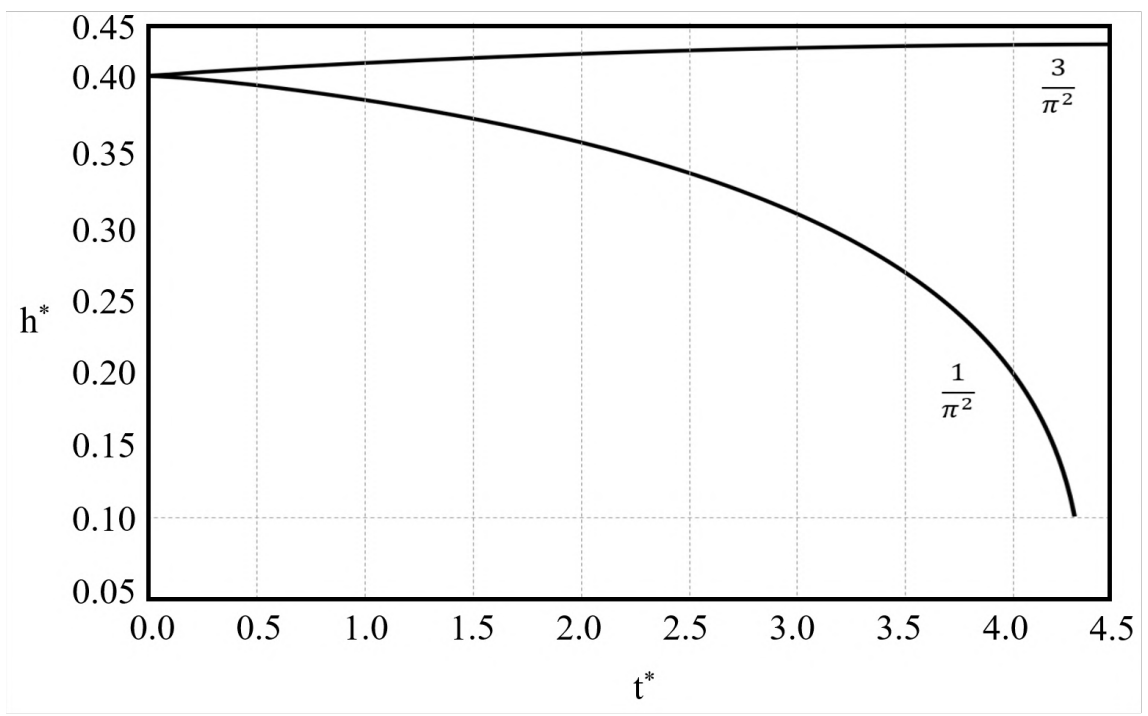

Figure 5.7: Evolution of the film thickness $h^{*}$ at $x=0$ for $S / A=1 / \pi^{2}$ and $S / A=3 / \pi^{2}$.

We introduce the term pinch velocity to refer to the velocity of which the free surfaces moves towards each other to facilitate the analysis. To that extent, the profile of the film thickness $h^{*}$ portrayed in Figure 5.7 for $S / A=1 / \pi^{2}$ indicates that the pinch velocity increases as the film rupture becomes imminent. Concerning $S / A=3 / \pi^{2}$, the perturbation amplitude of the thin film for the stability ratio $S / A=3 / \pi^{2}$ retracts up until $t^{*}=5.26$. Therefore, the case $S / A=3 / \pi^{2}$ portrays the behavior predicted by the linear stability criterion. On the other hand, the stability ratio $S / A=1 / \pi^{2}$ yields a rupture time of $t_{f}^{*}=4.3$ and behaves according to the prediction of the stability criterion. 


\subsection{2}

\section{Thin film rupture}

The rupture process is addressed under a perturbation of amplitude $\epsilon=0.1 H_{C}$ and ratio $S / A=1 / \pi^{2}$. At these conditions, the liquid sheet is unstable and ruptures in a finite time. As the film thins, nonlinear effects become more prominent and the drainage of the film increases. Figure 5.8 illustrates the temporal evolution of the free surface under the aforementined configuration.

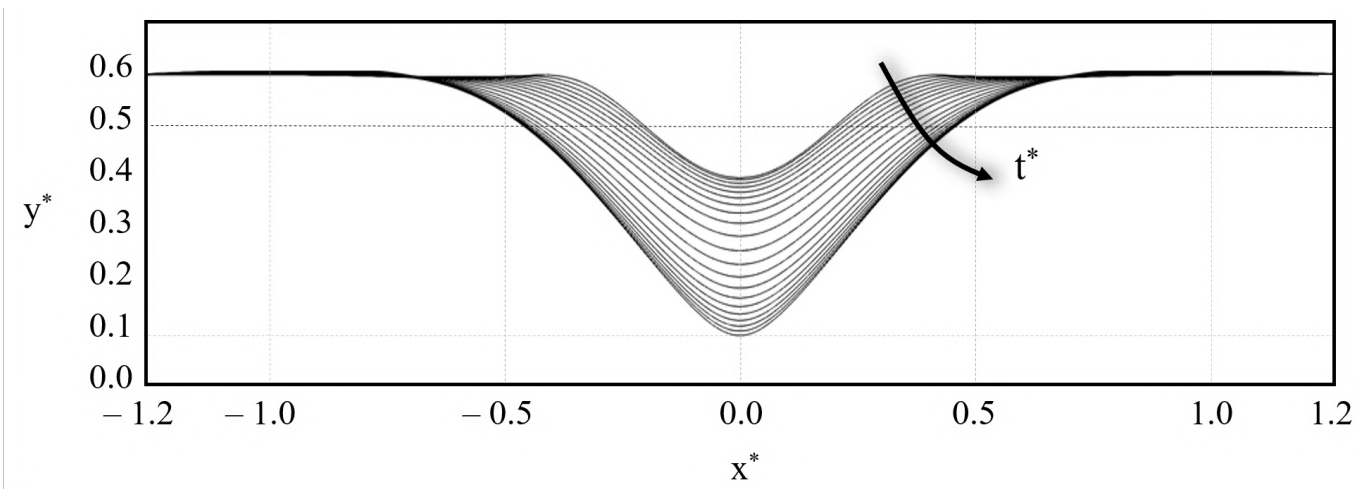

Figure 5.8: Temporal evolution of the free thin liquid film near the perturbed region.

The action of intermolecular van der Waals forces on the interfaces of the liquid film lead to a pressure gradient from the region of minimum thickness towards the sheet extremities. Figure 5.9 shows the dimensionless pressure distribution inside the domain of the thin liquid film at $t^{*}=0.1$. Initially, the pressure difference from $x^{*}=0$ to $x^{*}=1$ is around $\delta p^{*}=0.33$. Also, a local pressure gradient below the perturbed region of the free surface become apparent in the beginning of the temporal evolution.

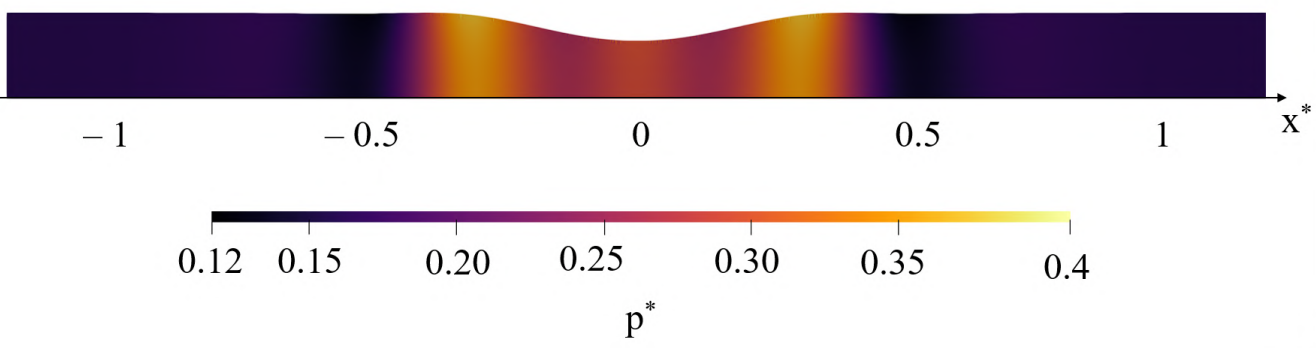

Figure 5.9: Bulk pressure distribution at $t^{*}=0.1$.

The stronger pressure gradient occurs from $x^{*}=0.4$ towards $x^{*}=1$ and it is symmetric with respect to $x^{*}=0$. This occurrence portrays the effects of capillarity and as expected, reaches its peaks in the maximum surface 
curvature points. However, as the curvature of the free surface decreases, these local variations in the pressure field become weak and no longer contribute to the system dynamics. Then, the bulk pressure at $x^{*}=0$ increases with time until the thin film reaches the critical value $h_{f}$, leading to a pressure difference of approximately $\delta p^{*}=12$ towards the outflow direction. The pressure field at $t^{*}=0,1,2,3.5$ and 4.3 is presented in Figure 5.10, respectively from top to bottom.

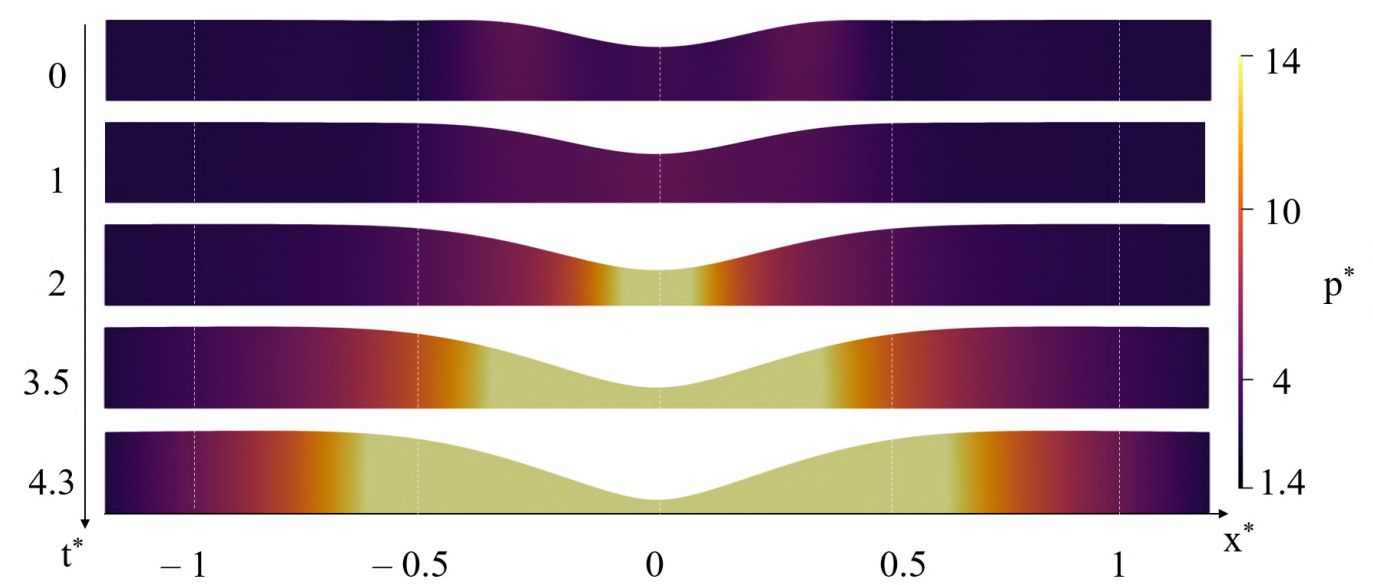

Figure 5.10: Bulk pressure field at $t^{*}=0,1,2,3.5$ and 4.3 .

The drainage of the thin liquid film is better analyzed by examining the $x$ component of the velocity field, shown in Figure 5.11. The flow occurs symmetrically in terms of $x^{*}=0$, which is an assumption used in the theoretical findings of Ida and Miksis [52]. As the film thins and the pressure gradient becomes stronger, the drainage velocity rises, as expected.

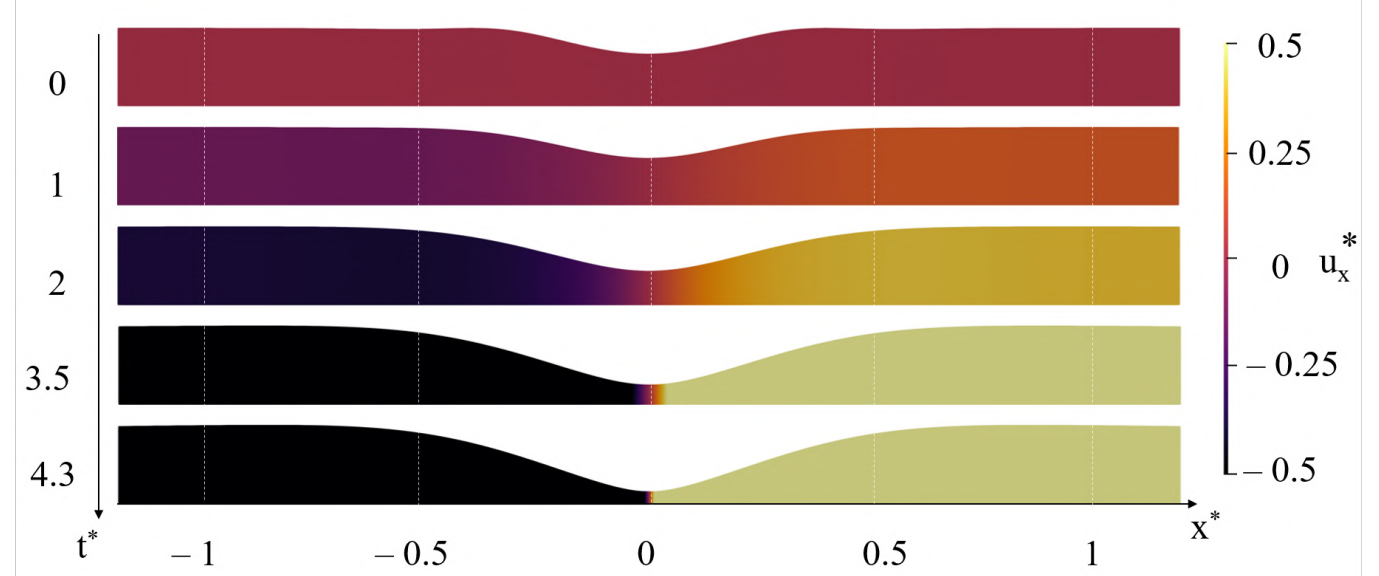

Figure 5.11: Temporal evolution of the $x$ component of the velocity field at $t^{*}=0,1,2,3.5$ and 4.3 . 
Figure 5.12 represents the evolution of the $y$ component of the velocity field. It is noticeable that the $y$ component of the velocity is mostly null across the domain, except near the region of minimum thickness. As discussed earlier, the nonlinear evolution of the sheet thickness may be due to an increase in the surface velocity, which are driven by the long range intermolecular attractions.

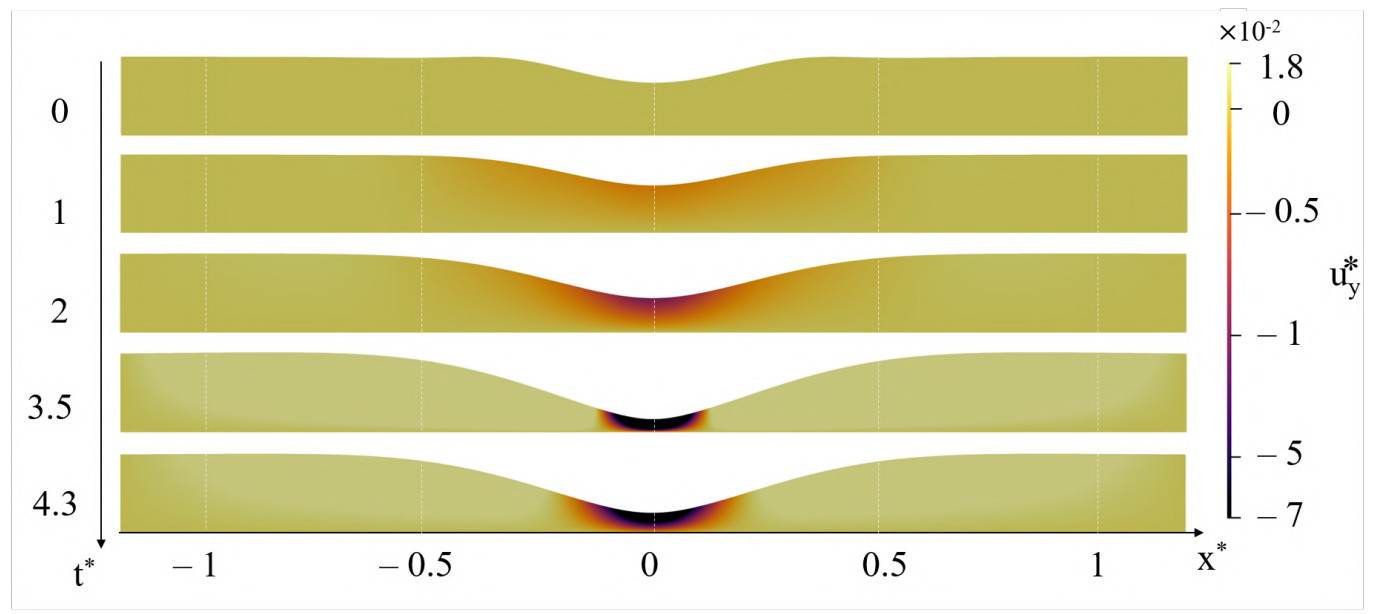

Figure 5.12: Time evolution of the $y$ component of the velocity field at $t^{*}=0,1,2,3.5$ and 4.3

As the thin liquid film undergoes rupture, the velocity of attraction between the free surfaces is maximum at $x^{*}=0$. Consequently, the free surfaces moving towards one another, squeezing the liquid film at $\left(x^{*}, y^{*}\right)=(0,0)$. The displacement of fluid away from the rupture causes an increase in the film thickness immediately near $x=0$, which may be related to the physical phenomenon of capillary ridges. Also at $x^{*}=0$, the $x$ component of the velocity field is equal to zero because of the flow symmetry and $y$ component is maximum, which resembles the behavior of a saddle point. Figure 5.13 portrays the velocity magnitude $\left\|\mathbf{u}^{*}\right\|=\sqrt{\left(u_{x}^{*}\right)^{2}+\left(u_{y}^{*}\right)^{2}}$ in the spatial vicinity of the rupture region at $t^{*}=0.1$.

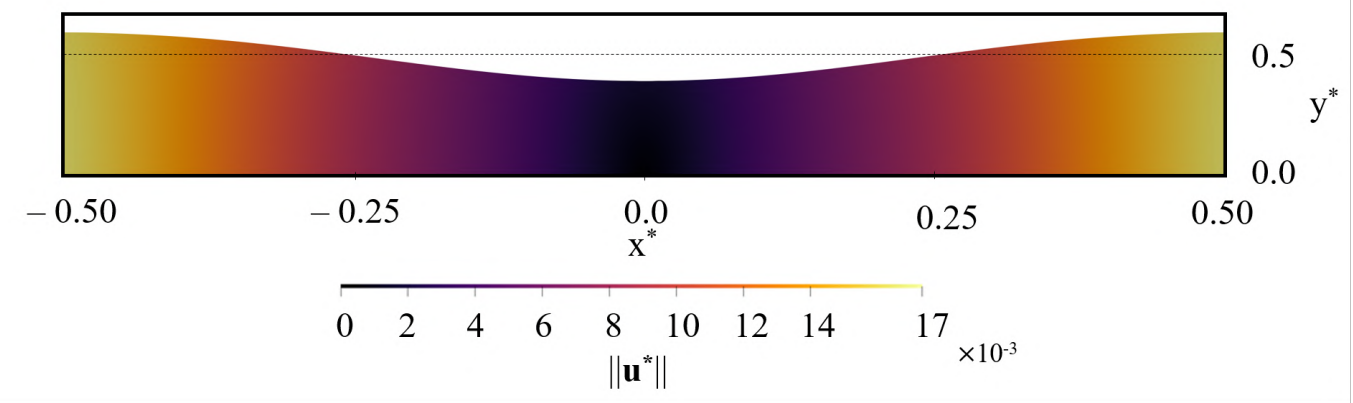

Figure 5.13: Velocity magnitude in the spatial vicinity of the rupture point at $t^{*}=0.1$. 
The behavior of the velocity magnitude depicted by Figure 5.13 continues as the rupture of the film evolves in time. The region present an analogous dynamic behavior throughout the entire rupture event. Figure 5.14 shows the velocity magnitude near the rupture region at rupture time $t_{r}^{*}=4.3$.

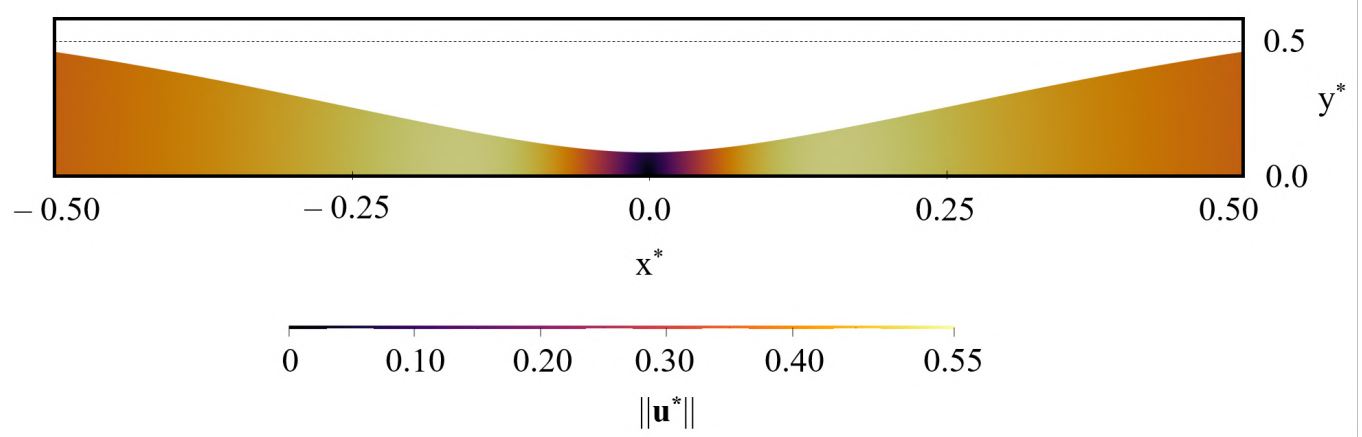

Figure 5.14: Velocity magnitude near the rupture point at $t^{*}=4.3$.

The $x$ and $y$ components of the velocity field along the free surface at $t^{*}=4.3$ is presented in Figure 5.15. The $x$ component of the surface velocity reaches its maximum value $u_{x}^{*}=0.52$ at $x^{*}=0.15$ and minimum value $u_{x}^{*}=-0.52$ at $x^{*}=-0.15$. Analogously, the $y$ component of the velocity field reaches the maximum value $u_{y}^{*}=0.018$ respectively at $x^{*}= \pm 0.275$ and minimum value $u_{y}^{*}=-0.076$ at $x^{*}=0$.

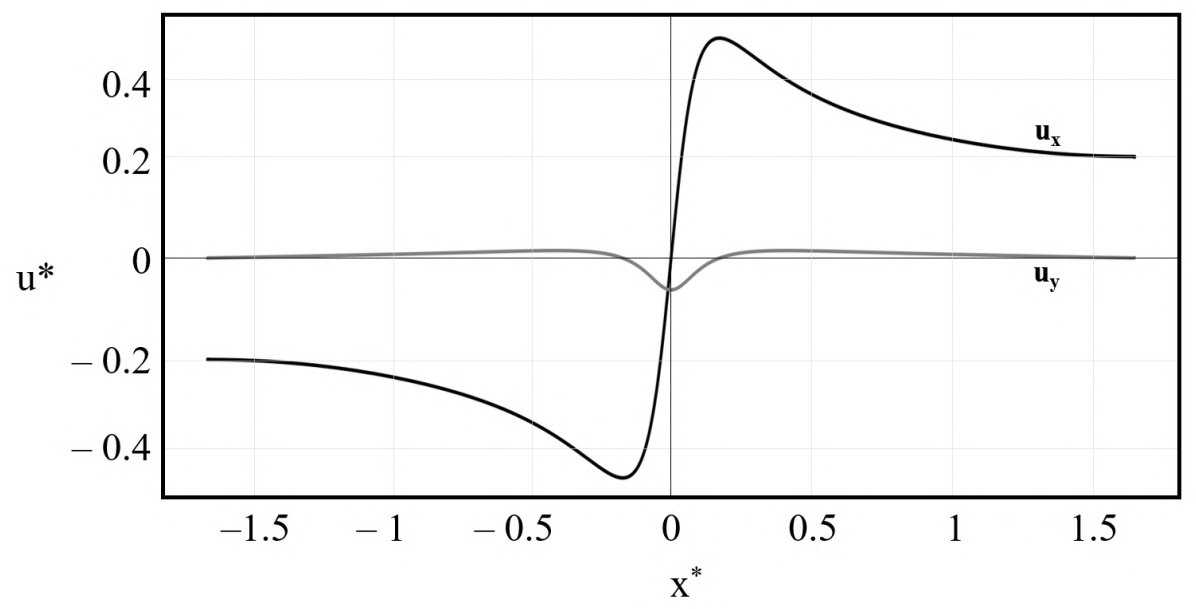

Figure 5.15: Surface $x$ and $y$ velocity components at $t^{*}=4.3$.

\section{3}

\section{Viscous interface}

The results presented in this section refer to the dynamics of thin liquid films with viscous interfaces. As discussed earlier, the Boussinesq number is the dimensionless number which measures the ratio of surface viscous forces 
to bulk viscous forces. We evaluate the action of interfacial viscosity on the rupture process sistematically for a fixed stability ratio of $S / A=1 / \pi^{2}$ and $0 \leq B o \leq 75$.

We aim at analyzing the effects interfacial rheology near the rupture, wherein the results obtained for $0 \leq B o \leq 75$ are used to provide a broad view on the system dynamics. Also, we implement an adaptative time step using $\overline{C o}=5000$ to evolve the system in time, as in the simple case.

\subsection{1}

\section{Amplitude growth}

Interfacial viscosity leads to extra interfacial stresses along the tangential direction of the interface, which affect the dynamics of thin liquid sheets. Interfacial viscosity introduces an additional resistance to the movement of the free surface, which is due to the presence of complex interfacial structures on the microscopic scale. The interfacial stresses oppose the interface deformation and delay the perturbation growth.

Figure 5.16 presents the evolution of the perturbation $\varepsilon(t)$ at $S / A=1 / \pi^{2}$ for $0 \leq B o \leq 75$. $B o=0$ corresponds to the simple interface case, shown before. At this value of $S / A$, the perturbation grows rapidly until breakup. For $B o \geq 15$, the growth of the perturbation decreases drastically, albeit still being nonzero.

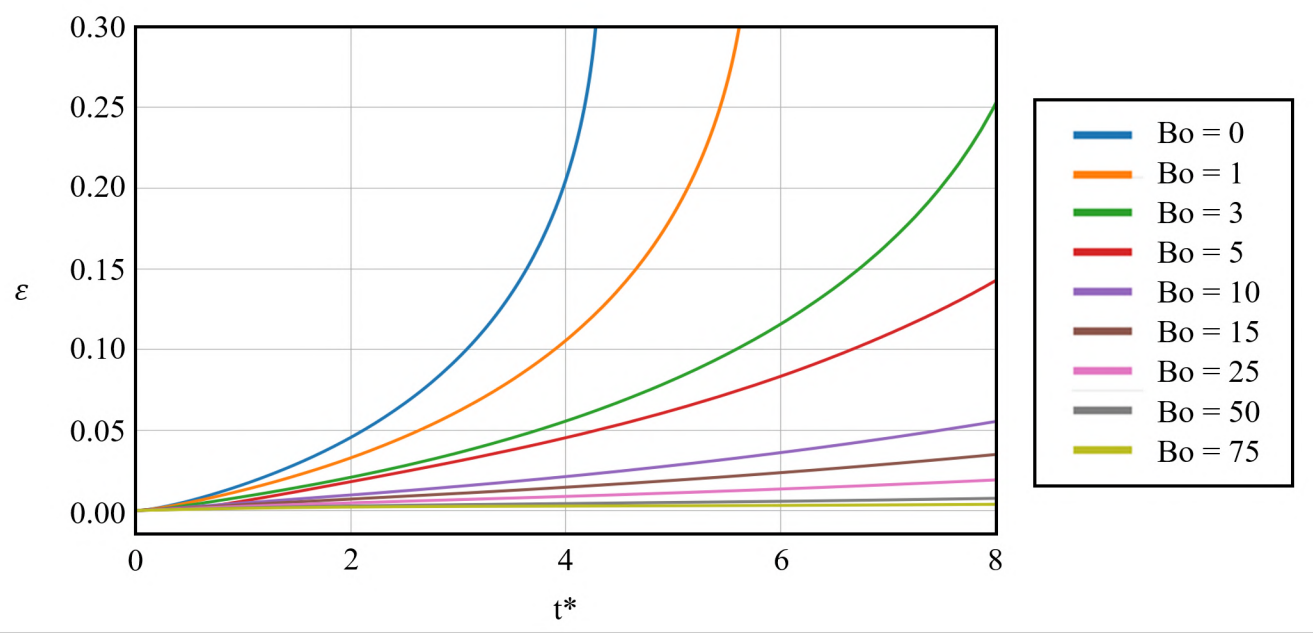

Figure 5.16: Evolution of the amplitude of the perturbation for $0 \leq B o \leq 75$.

The evolution of the film thickness at $x^{*}=0$ under the influence of interfacial viscosity is presented in Figure 5.17. The results indicate that the rupture process is slowed down as $B o$ increases. The sheet breakup time increases as Bo rises. 


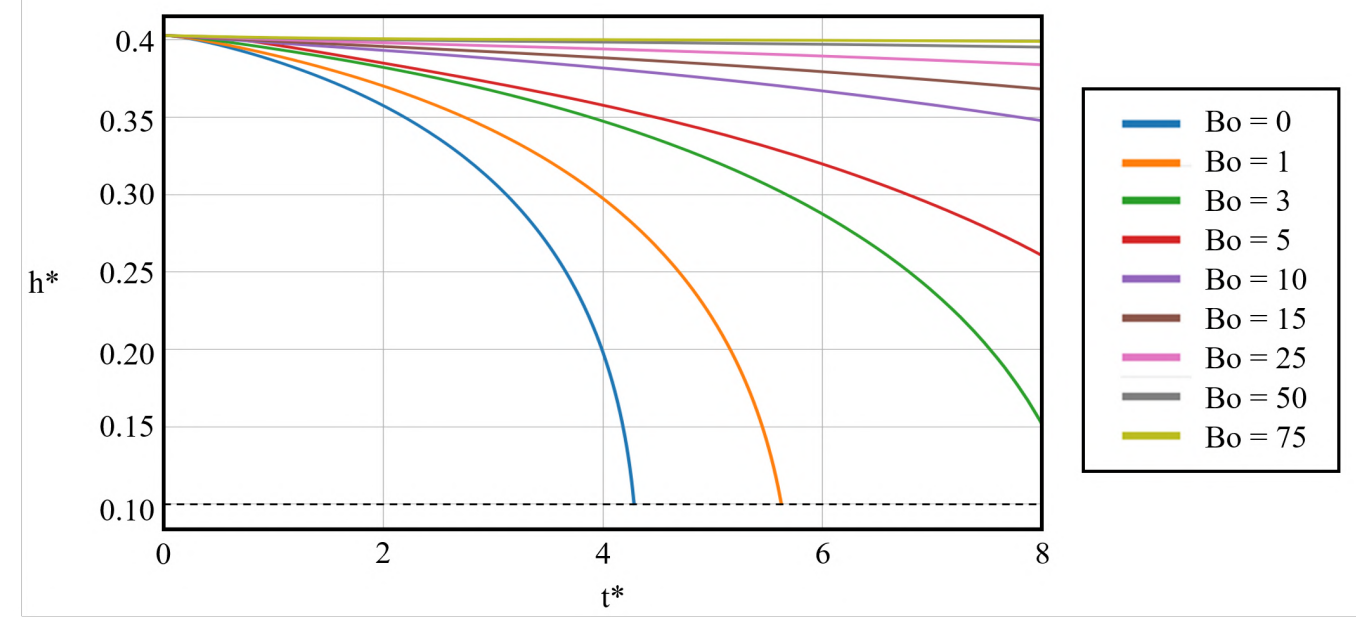

Figure 5.17: Film thickness evolution for $0 \leq B o \leq 75$.

Furthermore, Figure 5.18 presents the rupture time as a function of the Boussinesq number, for $0 \leq B o, \leq 5$.

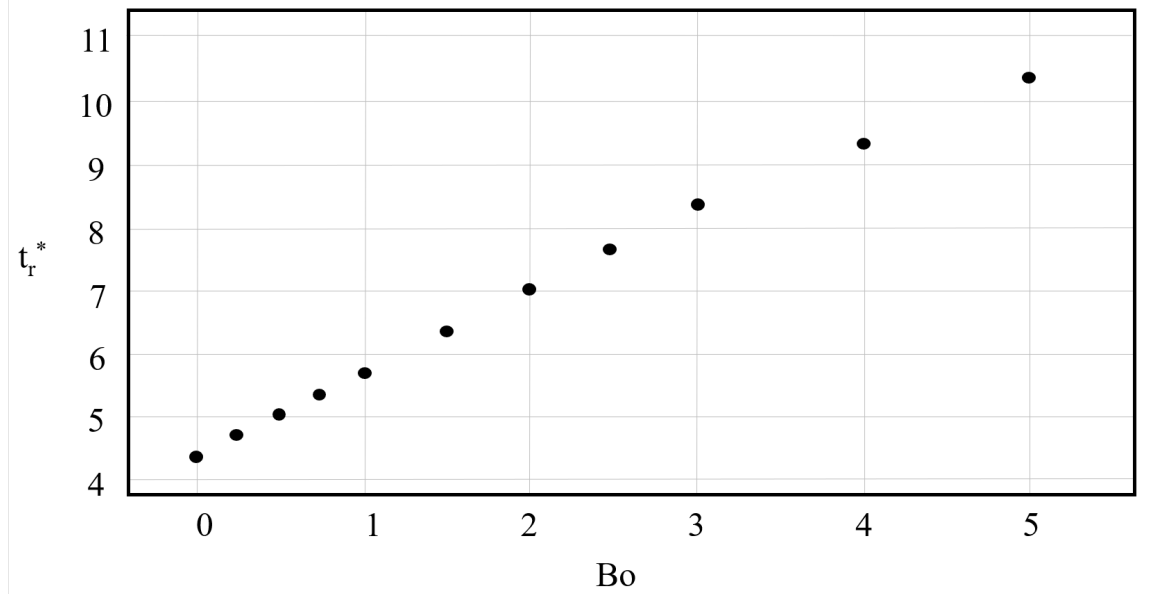

Figure 5.18: Rupture time as a function of the Boussinesq Number. 


\subsection{2}

\section{Velocity and pressure fields}

The configuration of the free surface near the perturbed region at the rupture time for $0 \leq B o \leq 5$ is illustrated in Figure 5.19. It is interesting to note that as the interfacial viscosity rises, the surface area at the breakup time becomes smaller.

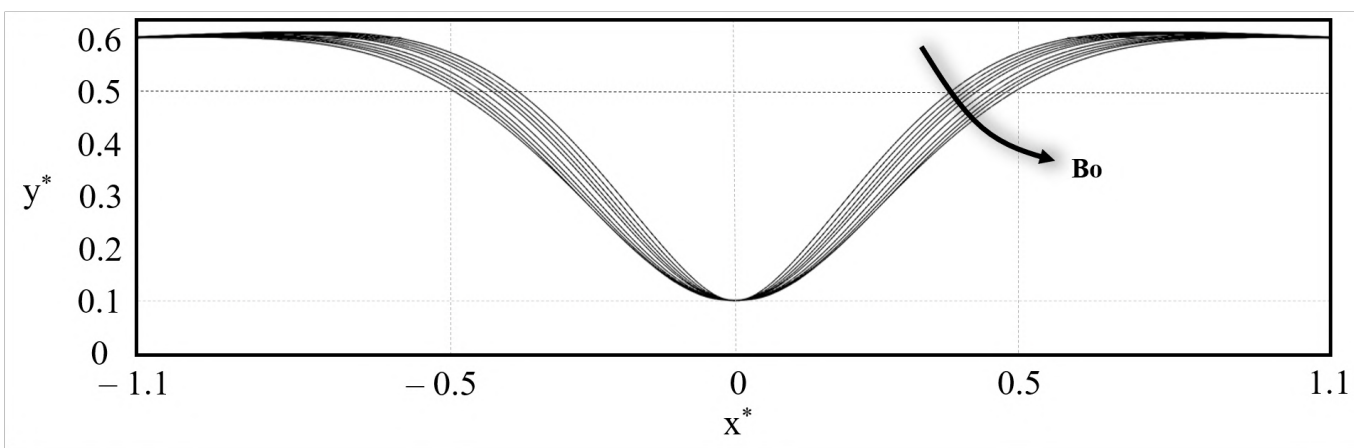

Figure 5.19: Configuration of the free surface at rupture time for $0 \leq B o \leq 5$.

Figure 5.20 portrays the pressure distribution over the domain at $t^{*}=1$ for $B o=0,5,15,25,50$ and 75 . We notice that the pressure distribution in the bulk at $t^{*}=1$ is similar for all the values of $B o$, as the system hasn't reached the temporal vicinity of rupture. However, the thickness evolution slows down as we increase $B o$, as previously reported in Figures 5.16, 5.17 and 5.18.

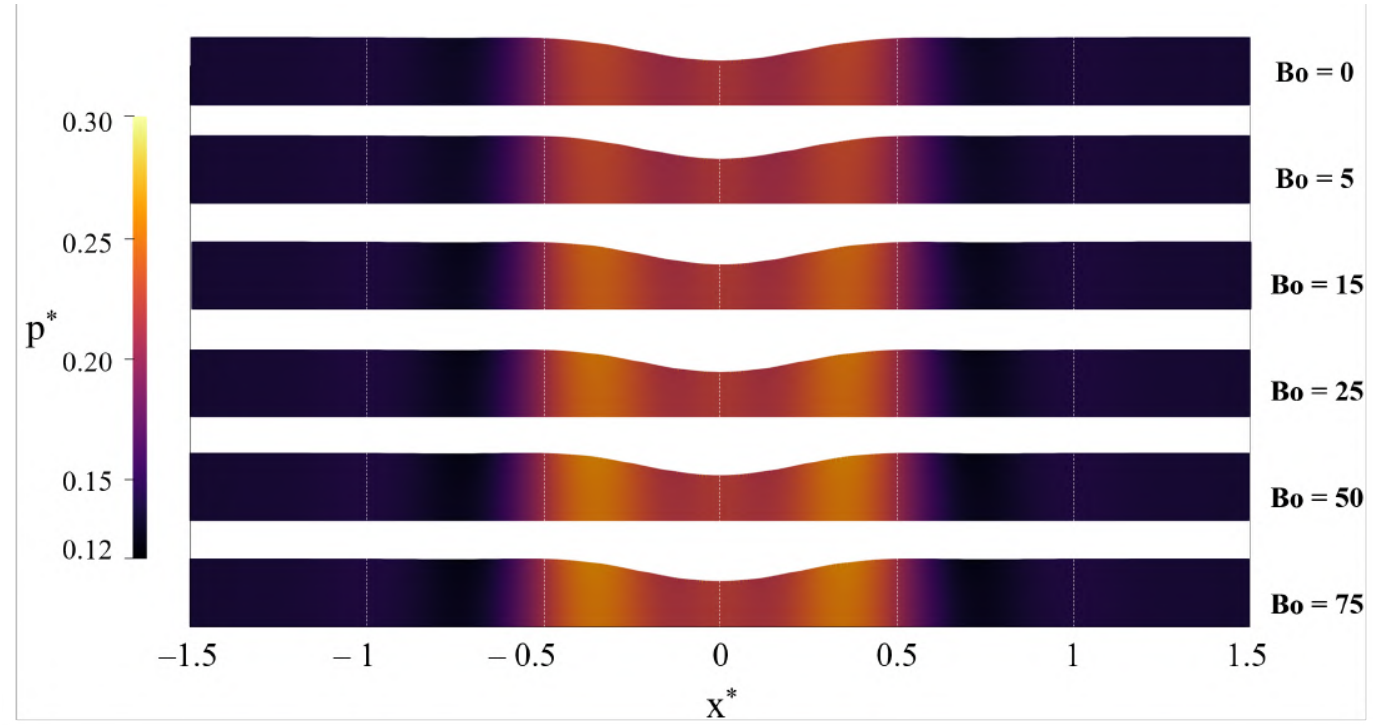

Figure 5.20: Pressure distribution at $t^{*}=1$ for $B o \in[0,75]$.

Moreover, the velocity field in the domain is clearly influenced by the effects of surface viscosity. Figures 5.21 and 5.22 show the $x$ and $y$ components of the velocity field at $t^{*}=1$ for $B o=0,5,15,25,50$ and 75 . The effects of 
interfacial rheology in the early dynamics of the system result in a reduction of the velocity gradient along the interface, which leads to a reduction on the bulk velocity.

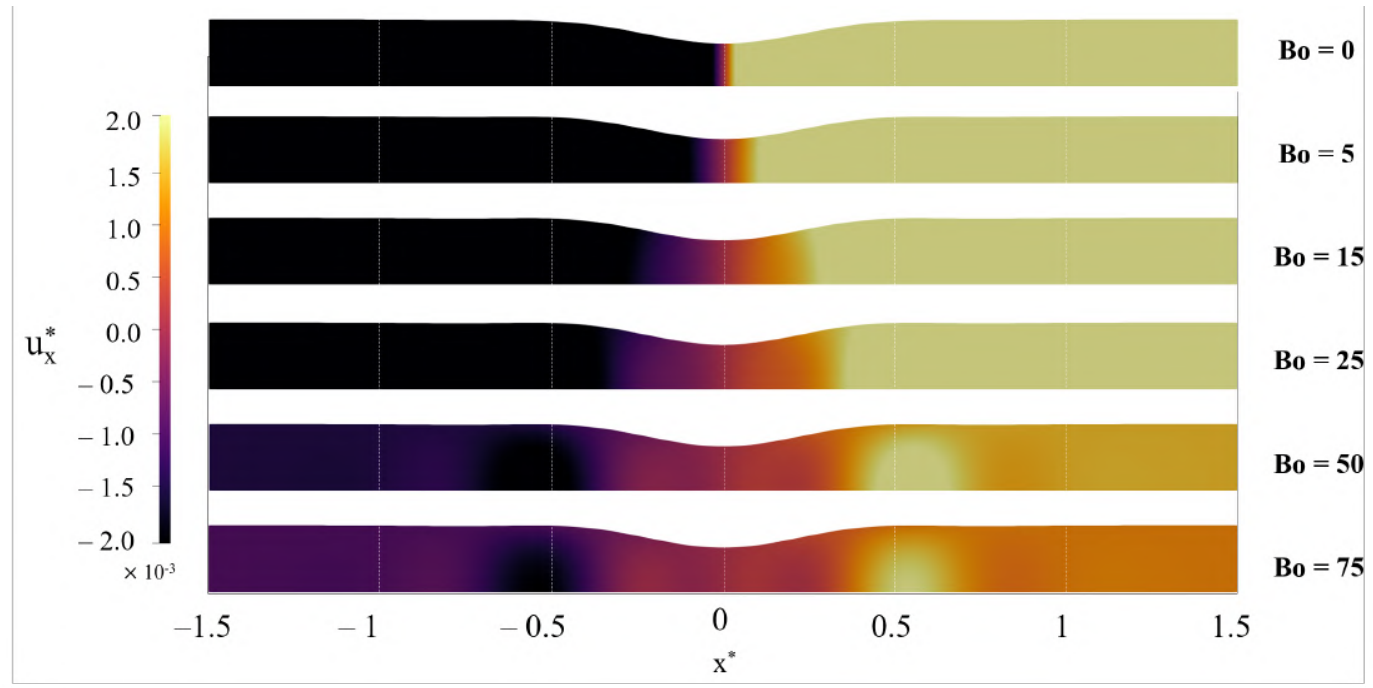

Figure 5.21: $x$ component of the velocity field at $t^{*}=1$ for $B o \in[0,75]$.

The reduction of the $x$ component of the velocity occurs close to the surface and near $x^{*}=0.5$ and $x^{*}=-0.5$. Furthermore, Figure 5.22 indicates that the increment of interfacial viscosity reduces the value of the $y$ component of the velocity field at $x^{*}=0$.

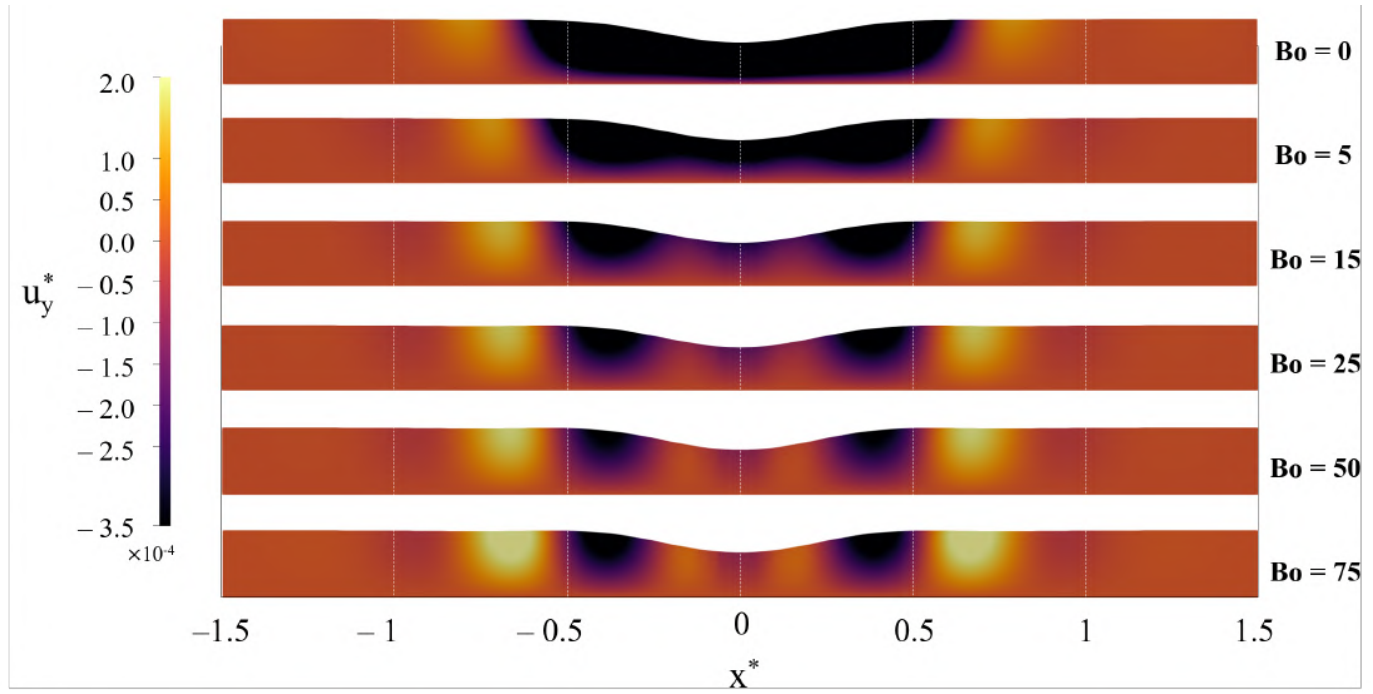

Figure 5.22: $y$ component of the velocity field at $t^{*}=1$ for $B o \in[0,75]$.

The results presented in Figures 5.21 and 5.22 also indicate that the additional effects of interfacial rheology reduces the drainage rate of the film. As the long-range intermolecular attraction between the free surface occurs, the effects of interfacial viscosity slow down the surface movement. Figures 5.23, 
5.24 and 5.25 illustrate the behavior of the pressure and $x$ and $y$ components of the velocity field at $t^{*}=4.3$, respectively. All these cases are presented using the same scale to ease the comparison.

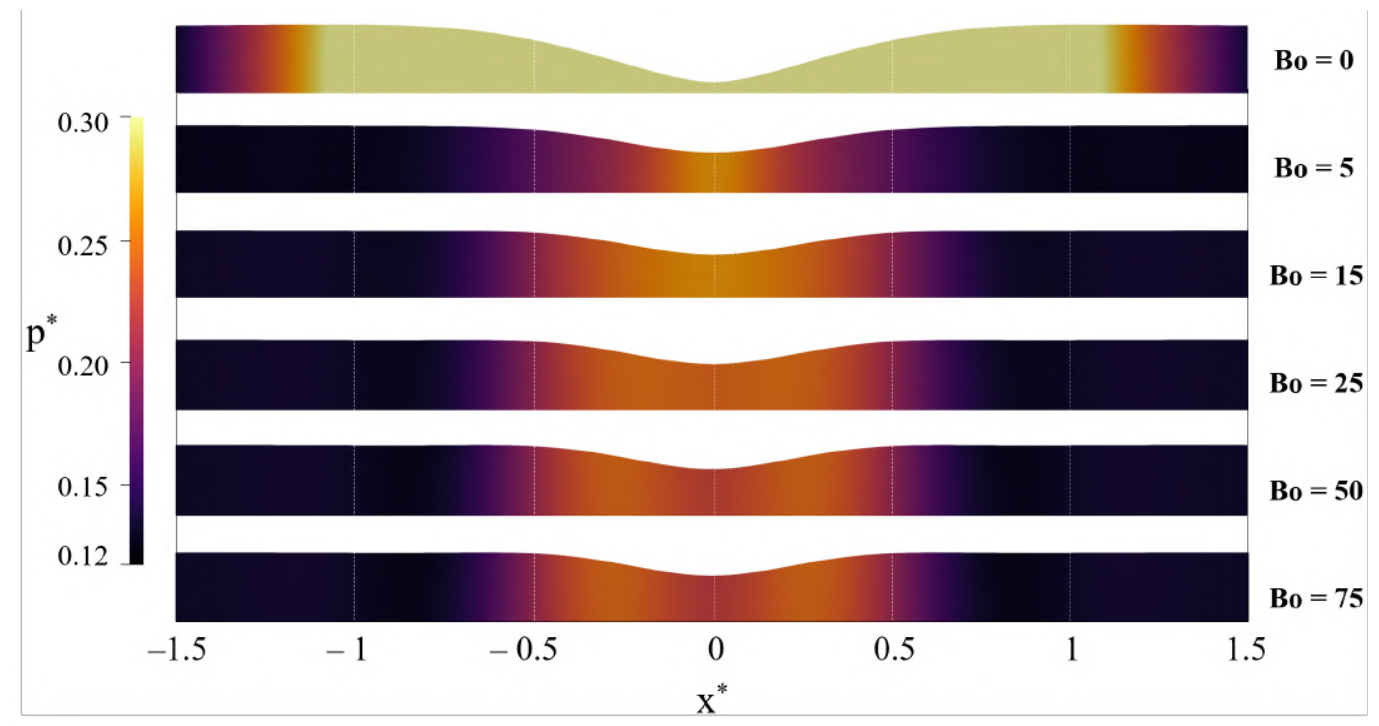

Figure 5.23: Pressure distribution at $t^{*}=4.3$ for $B o \in[0,75]$.

The evolution of the pressure field occur analogously to the simple case. The results portrayed in Figure 5.24 also indicate the reduction of the drainage rate, which may be accounted for the delay on the rupture process. Likewise, the result presented in Figure 5.25 indicates a strong reduction of the $y$ component of the velocity field.

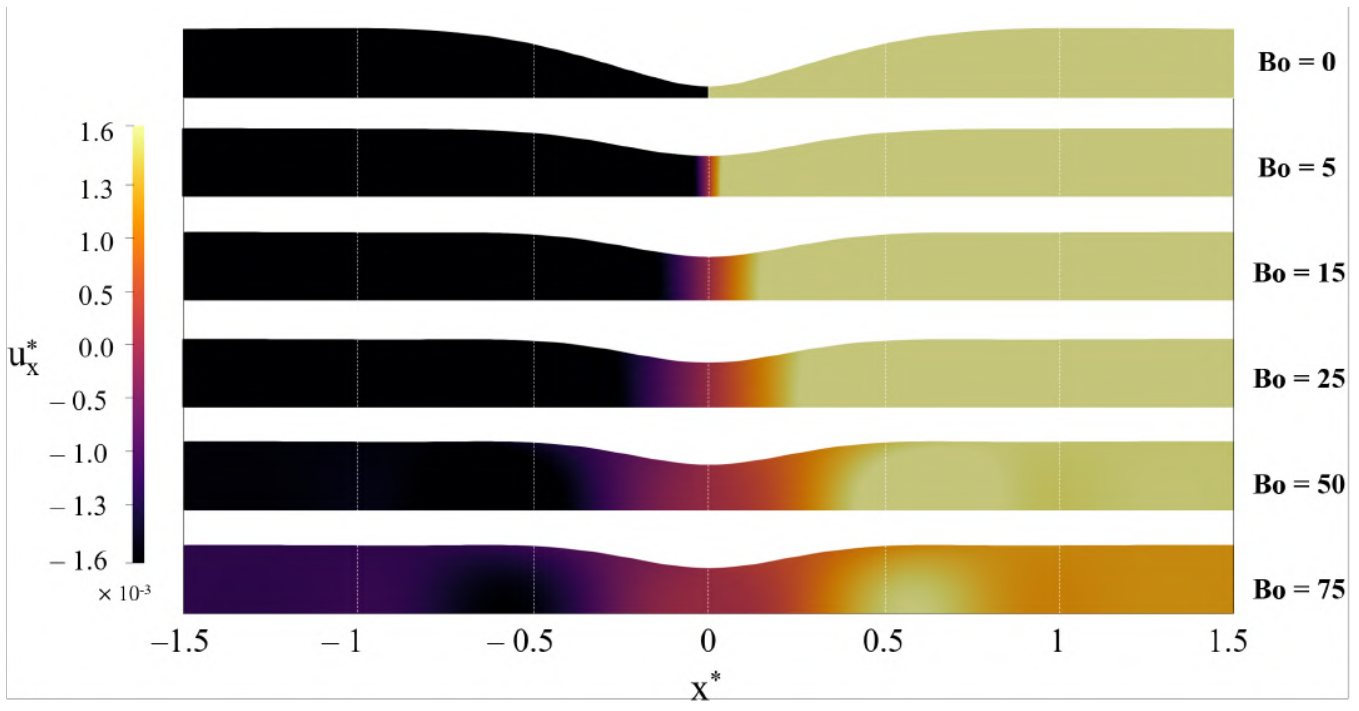

Figure 5.24: $x$ component of the velocity field at $t^{*}=4.3$ for $B o \in[0,75]$.

As the additional effects of interfacial viscosity oppose the intermolecular attraction between the free surface, the drainage of the film becomes slower. 
Also, the pinch velocity is reduced as the Boussinesq number increases.

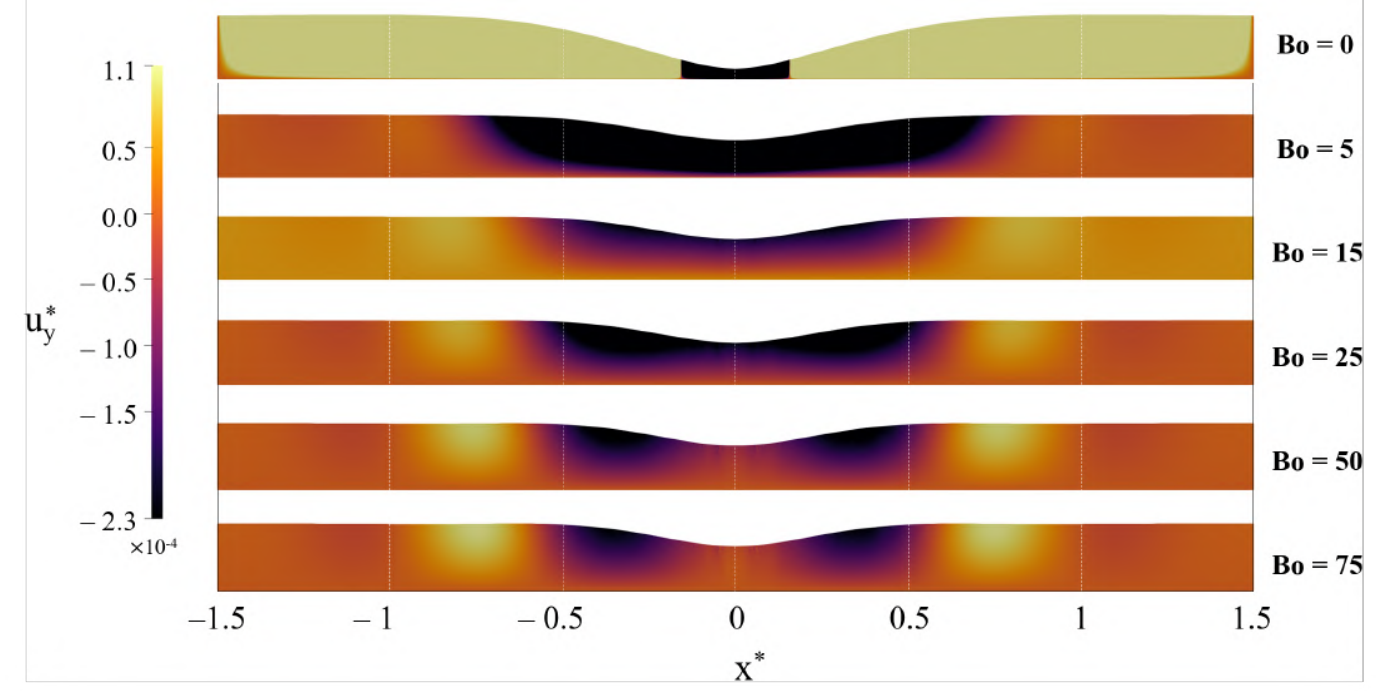

Figure 5.25: $y$ component of the velocity field at $t^{*}=4.3$ for $B o \in[0,75]$.

Thus, the amount of fluid displaced from the perturbed region of the bulk towards the extremities is consequently reduced. The $x$ and $y$ components of the surface velocity at the rupture times presented in Figure 5.18 are illustrated in Figure 5.26.

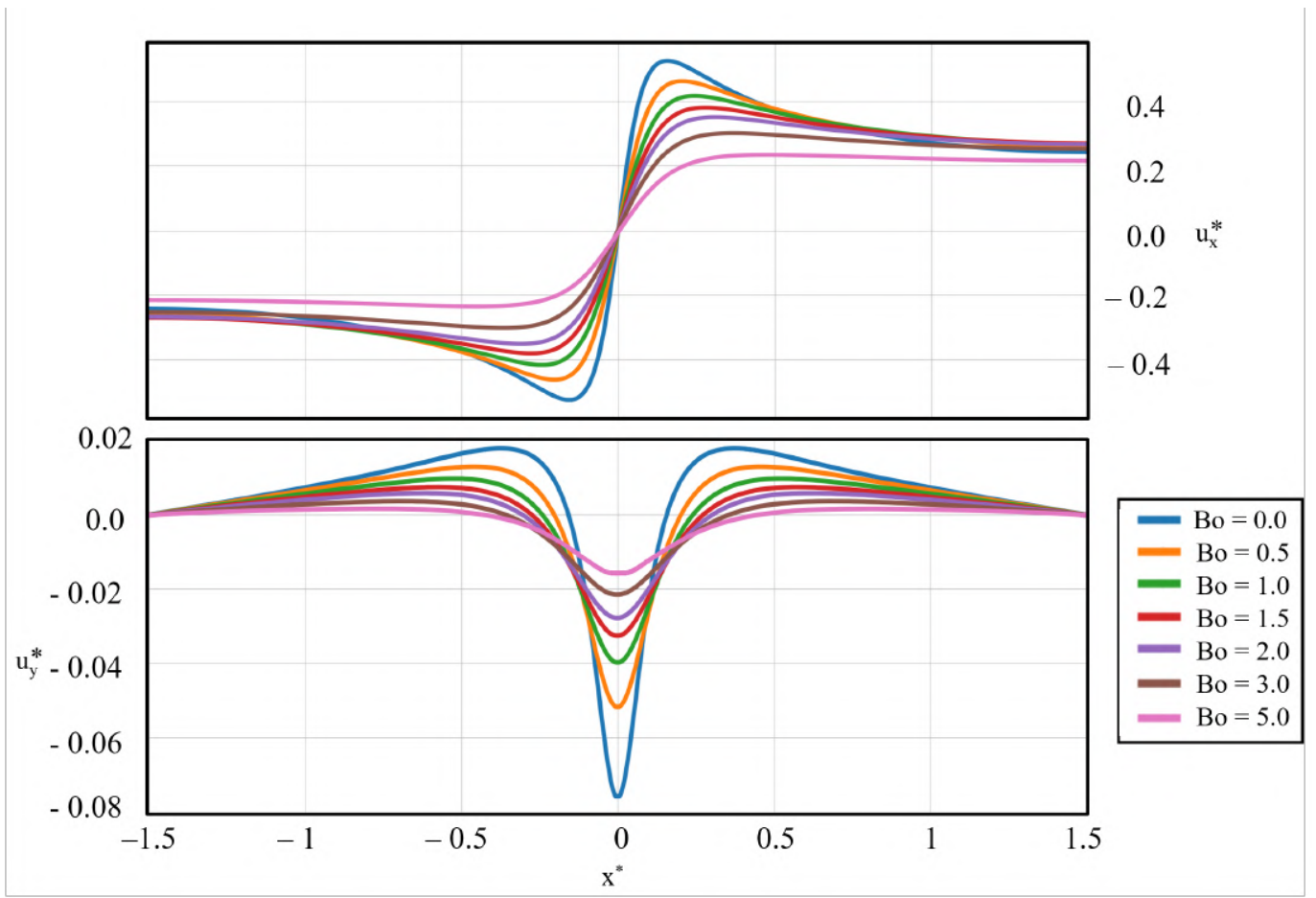

Figure 5.26: Surface $x$ and $y$ velocity components at rupture time for $0 \leq B o \leq$ 5 . 
With the increment of the interfacial viscosity, the mobility of the free surface is reduced in both $x$ and $y$ directions. Hence, the long-range intermolecular van der Waals forces are strongly opposed by the extra viscous stresses. The profile of the surface velocity presented in Figure 5.26 indicates a reduction of surface mobility near $x^{*} \pm 0.5$. 


\section{6 \\ Conclusion}

We presented a numerical investigation of the rupture dynamics of stationary free thin liquid films with viscous interfaces. We employed a 2D model of the domain with a symmetry line and used conservation of mass and linear momentum to tackle the dynamics of thin liquid films. To account for the extra viscous stress along the free surface of the film, the Boussinesq-Scriven constitutive model was implemented.

The thin liquid film was taken out of the equilibrium position by an initial perturbation shaped as a sine wave. The set of differential equations was simulated using the Finite Element Method implemented in Python by the FEniCS framework and the displacement of the interface was handled with the ALE method.

The long-range intermolecular van der Waals forces play a dominant role on the system dynamics, which is accounted by our modelling through a surface force referred as disjoining pressure. The effects of capillarity and interfacial viscosity oppose the action of the disjoining pressure, and according to the stability criterion proposed by Erneux and Davis [38], the ratio $S / A$ defines whether the system recovers from the intermolecular attraction between the free surfaces or undergoes a finite time rupture.

We evaluated the behavior of a simple interface under different conditions of the stability ratio. Concerning our findings, the stability criterion succeeds at predicting the early behavior of the system but fails to accurately describe the temporal evolution of the free thin liquid film after a given time. This occurrence may be due to the fact that the stability criterion does not account for the nonlinear terms of the governing equations, which can lead to false predictions when nonlinear effects become prominent on the system dynamics. Nonetheless, the stability criterion provides thoughtful insights on the early dynamics of the thin liquid film.

The numerical solution of the system also revealed that the effects of interfacial rheology strongly opposes the action of the disjoining pressure. The effects of interfacial rheology were analyzed by varying the Boussinesq Number $B o$ while maintaining constant all other parameters. The results obtained show that for the range $0 \leq B o \leq 5$, the rupture delay behaves almost linearly with the surface viscosity. Also, the mobility of the free surface is highly affected by surface viscosity, meaning that the increase of $B o$ reduces the magnitude of the surface velocity. 
By including interfacial viscosity, the growth of perturbation decelerated and the rupture slowed down with a flatter interface. Moreover, the effects of rheology have been accounted for the delay of the growth of perturbations in free thin liquid films in the literature. To that extent, the effect of interfacial rheology may also be accounted for the rupture delay, as the interfacial state of the fluid is modeled by an approach similar to that of rheollogically complex fluids.

\section{1}

\section{Future works}

We studied the effects of interfacial viscosity on the breakup of thin liquid films for $S / A=1 / \pi^{2}$, which indicates that the reduction of surface mobility reduces the drainage rate of the liquid film. However, one may ask whether the action of interfacial viscosity could delay the evolution of out-ofequilibrium thin liquid sheets represented by $S / A \geq 2 / \pi^{2}$. The linear stability criterion predicts a sheet recovery under these configuration, which may also be deccelerated due to the reduction of surface mobility.

The effects of interfacial rheology were evaluated in a 2D configuration under a symmetric perturbation and provided insights on the interplay between surface forces and the hydrodynamics of a Newtonian bulk. This approach may also be useful to numerically analyze the hydrodynamics of viscoelastic bulks. Surfactant transport across the interface may also be included in the system dynamics, provided a constitutive model for the temporal evolution of the surfactant concentration. The latter could lead to interesting results regarding the action of Marangoni convection on the interplay between interface forces and the disjoining pressure. Furthermore, the implementation of a 3D model using the FEniCS framework is straightforward and could showcase visual insights on the dynamics of thin liquid films.

The evolution of asymmetric perturbations on the free surfaces of the thin liquid film may also reveal several interesting behaviors. The absence of a symmetry plane in the formulation could yield generalized results such as the occurrence of both squeezing and buckling of the liquid film during the rupture dynamics.

The latest developments of the FEniCS framework provided an update approach to treat complex boundary conditions, namely the Meshview functionality. The use of Meshview could improve the accuracy of the numerical solutions while reducing the occurrence of spurious oscillations in the results, as the tool provides a straightforward approach to deal with complex conditions over boundaries. 
We emphasize the functionality of the FEniCS platform mainly due to its open-source character and multi-purpose nature. The work developed in this manuscript relies on the dynamics of stationary thin liquid films, which could be extended for studies that account for more realistic descriptions of the physical phenomena, such as moving liquid curtains and other well behaved free surface phenomena. $\times$ 


\section{Bibliography}

[1] R. V. Craster and O. K. Matar. Dynamics and stability of thin liquid films. Reviews of Modern Physics, 81(3):1131-1198, 2009.

[2] Michael Angelo Y.H. Lam, Linda J. Cummings, and Lou Kondic. Stability of thin fluid films characterised by a complex form of effective disjoining pressure. Journal of Fluid Mechanics, 841(2003):925-961, 2018.

[3] Gioele Mariano Nicolò Balestra. Pattern formation in thin liquid films: from coating-flow instabilities to microfluidic droplets, 2018.

[4] L. Zhong, C. F. Ketelaar, R. J. Braun, C. G. Begley, and P. E. KingSmith. Mathematical modelling of glob-driven tear film breakup. Mathematical Medicine and Biology, 36(1):55-91, 2019.

[5] J. B. Grotberg. Pulmonary flow and transport phenomena. Annual Review of Fluid Mechanics, 26(1):529-571, 1994.

[6] N. J. Dudney and B. J. Neudecker. Solid state thin-film lithium battery systems. Current Opinion in Solid State and Materials Science, 4(5):479482, 1999.

[7] Mahmoud Abdelfatah, Johannes Ledig, Abdelhamid El-Shaer, Alexander Wagner, Azat Sharafeev, Peter Lemmens, Mohsen Mohamed Mosaad, Andreas Waag, and Andrey Bakin. Fabrication and characterization of flexible solar cell from electrodeposited $\mathrm{Cu} 2 \mathrm{O}$ thin film on plastic substrate. Solar Energy, 122:1193-1198, 2015.

[8] Melisa Becerra and Marcio S. Carvalho. Stability of viscoelastic liquid curtain. Chemical Engineering and Processing: Process Intensification, 50(5-6):445-449, 2011.

[9] M. S. Bazzi and M. S. Carvalho. Effect of viscoelasticity on liquid sheet rupture. Journal of Non-Newtonian Fluid Mechanics, 264:107-116, 2019.

[10] Close up of an eye. http://imgur.com/gallery/Krc2iG3. Accessed: 2021-07-20.

[11] Dramatic lava flow in hawaii. https://www.cbsnews.com/pictures/ dramatic-lava-flow-in-hawaii/. Accessed: 2021-07-20. 
[12] Yasunori Takeda, Kazuma Hayasaka, Rei Shiwaku, Koji Yokosawa, Takeo Shiba, Masashi Mamada, Daisuke Kumaki, Kenjiro Fukuda, and Shizuo Tokito. Fabrication of Ultra-Thin printed organic TFT CMOS logic circuits optimized for low-voltage wearable sensor applications. Scientific Reports, 6:1-9, 2016.

[13] Mohar Dey, Atul S. Vivek, Harish N. Dixit, Ashutosh Richhariya, and James J. Feng. A model of tear-film breakup with continuous mucin concentration and viscosity profiles. Journal of Fluid Mechanics, 858:352-376, 2019.

[14] Gesundheit industrie on the health of bronchial tress. https://www. gesundheitsindustrie-bw.de/. Accessed: 2021-07-20.

[15] M. Pepicelli, N. Jaensson, C. Tregouët, B. Schroyen, A. Alicke, T. Tervoort, C. Monteux, and J. Vermant. Surface viscoelasticity in model polymer multilayers: From planar interfaces to rising bubbles. Journal of Rheology, 63(5):815-828, 2019.

[16] Nampueng Pangpaiboon, Rakchart Traiphol, and Nisanart Traiphol. Enhancing the stability of polystyrene ultrathin films by using star-shape polymers as dewetting inhibitors. Journal of Coatings Technology and Research, 12(6):1173-1183, 2015.

[17] Eline Hermans, M. Saad Bhamla, Peter Kao, Gerald G. Fuller, and Jan Vermant. Lung surfactants and different contributions to thin film stability. Soft Matter, 11(41):8048-8057, 2015.

[18] Maria Ekimova, Wilson Quevedo, Manfred Faube, Philippe Wernet, and Erik T.J. Nibbering. A liquid flatjet system for solution phase soft-x-ray spectroscopy. Structural Dynamics, 2(5), 2015.

[19] Jake D. Koralek, Jongjin B. Kim, Petr Brůža, Chandra B. Curry, Zhijiang Chen, Hans A. Bechtel, Amy A. Cordones, Philipp Sperling, Sven Toleikis, Jan F. Kern, Stefan P. Moeller, Siegfried H. Glenzer, and Daniel P. DePonte. Generation and characterization of ultrathin freeflowing liquid sheets. Nature Communications, 9(1):1-8, 2018.

[20] David J. Collins, Ofer Manor, Andreas Winkler, Hagen Schmidt, James R. Friend, and Leslie Y. Yeo. Atomization off thin water films generated by high-frequency substrate wave vibrations. Physical Review E - Statistical, Nonlinear, and Soft Matter Physics, 86(5), 2012. 
[21] Seungjun Chung, Kyungjune Cho, and Takhee Lee. Recent Progress in Inkjet-Printed Thin-Film Transistors. Advanced Science, 6(6), 2019.

[22] Rhutesh K. Shah, Ho Cheung Shum, Amy C. Rowat, Daeyeon Lee, Jeremy J. Agresti, Andrew S. Utada, Liang Yin Chu, Jin Woong Kim, Alberto Fernandez-Nieves, Carlos J. Martinez, and David A. Weitz. Designer emulsions using microfluidics. Materials Today, 11(4):18-27, 2008.

[23] Priscila Gonçalves Vasconcelos Sampaio, Mario Orestes Aguirre González, Paula de Oliveira Ferreira, Priscila da Cunha Jácome Vidal, Jonathan Paulo Pinheiro Pereira, Helder Rodrigues Ferreira, and Pedro Carlos Oprime. Overview of printing and coating techniques in the production of organic photovoltaic cells. International Journal of Energy Research, 44(13):9912-9931, 2020.

[24] Short course on atomization and sprays. https://ilasseurope.org/ short-course-on-atomization-and-sprays-february-15-18-2016-darmstadt/. Accessed: 2021-07-20.

[25] Alireza Mohammad Karim, Wieslaw J. Suszynski, William B. Griffith, Saswati Pujari, Lorraine F. Francis, and Marcio S. Carvalho. Effect of viscoelasticity on stability of liquid curtain. Journal of Non-Newtonian Fluid Mechanics, 257:83-94, 2018.

[26] Satoru Izumisawa and Myung S. Jhon. Stability analysis and molecular simulation of nanoscale lubricant films with chain-end functional groups. Journal of Applied Physics, 91(10 I):7583-7585, 2002.

[27] Makoto Mizukami, Shinya Oku, Seung Il Cho, Masahiro Tatetsu, Miho Abiko, Masashi Mamada, Tomo Sakanoue, Yoshiyuki Suzuri, Junji Kido, and Shizuo Tokito. A Solution-Processed Organic Thin-Film Transistor Backplane for Flexible Multiphoton Emission Organic Light-Emitting Diode Displays. IEEE Electron Device Letters, 36(8):841-843, 2015.

[28] Vincenzo Fiore, Student Member, Placido Battiato, Sahel Abdinia, Stephanie Jacobs, Egidio Ragonese, Senior Member, Giuseppe Palmisano, and Senior Member. complementary TFT technology on flexible substrate An Integrated 13 .56 - MHz RFID Tag in a Printed Organic Complementary TFT Technology on Flexible Substrate. IEEE Transactions on Circuits and Systems-I: regular Papers, 62(6):1668$1678,2015$. 
[29] Ahmed Esmail Shalan. Challenges and approaches towards upscaling the assembly of hybrid perovskite solar cells. Materials Advances, 1(3):292309, 2020.

[30] Akihiro Kojima, Kenjiro Teshima, Yasuo Shirai, and Tsutomu Miyasaka. Organometal halide perovskites as visible-light sensitizers for photovoltaic cells. Journal of the American Chemical Society, 131(17):60506051, 2009.

[31] Jeong Hyeok Im, Chang Ryul Lee, Jin Wook Lee, Sang Won Park, and Nam Gyu Park. 6.5 Nanoscale, 3(10):4088-4093, 2011.

[32] Nam Gyu Park and Kai Zhu. Scalable fabrication and coating methods for perovskite solar cells and solar modules. Nature Reviews Materials, 5(5):333-350, 2020.

[33] Atsushi Sakuda, Kentaro Kuratani, Mari Yamamoto, Masanari Takahashi, Tomonari Takeuchi, and Hironori Kobayashi. All-Solid-State Battery Electrode Sheets Prepared by a Slurry Coating Process. Journal of The Electrochemical Society, 164(12):A2474-A2478, 2017.

[34] Nurbol Tolganbek, Almagul Mentbayeva, Nurassyl Serik, Nursaule Batyrgali, Miras Naizakarayev, Kiyoshi Kanamura, and Zhumabay Bakenov. Design and preparation of thin film gel polymer electrolyte for 3D Li-ion battery. Journal of Power Sources, 493:229686, 2021.

[35] S. A. Shain and J.M. Prausnitz. Thermodynamics and Interfacial Tension of Multicomponent Liquid-Liquid Interfaces. J. Am. Chem. SOC., 65, and J. McMicking, Am. Petrol. Inst. Rasearch Report. 8. Thodos, George, A.1.Ch.E. Journal, 9(3):182, 1964.

[36] J. N. Israelachvili. Intermolecular and surface forces. Academic press, 2011.

[37] M. A. Carrozza, M. A. Hulsen, and P. D. Anderson. Benchmark solutions for flows with rheologically complex interfaces. Journal of NonNewtonian Fluid Mechanics, 286, 2020.

[38] Thomas Erneux and Stephen H. Davis. Nonlinear rupture of free films. Physics of Fluids A, 5(5):1117-1122, 1992.

[39] Dimitri Vaynblat, John R. Lister, and Thomas P. Witelski. Rupture of thin viscous films by van der waals forces: Evolution and self-similarity. Physics of Fluids, 13(5):1130-1141, 2001. 
[40] A. Sheludko. Thin liquid films. Advances in Colloid and Interface Science, (T49B):519-524, 1967.

[41] Martine Prévost and Dominique Gallez. Nonlinear rupture of thin free liquid films. The Journal of Chemical Physics, 84(7):4043-4048, 1985.

[42] Saad M. Bhamla, Chew Chai, Marco A. Àlvarez-Valenzuela, Javier Tajuelo, and Gerald G. Fuller. Interfacial mechanisms for stability of surfactant-laden films. PLoS ONE, 12(5):1-14, 2017.

[43] B. V. Derjaguin and E. Obukov. Anomalien dinner flussigkeits- schichten iii. Acta Physicochim U.R.S.S., 5:1-22, 1936.

[44] B. V. Derjaguin and I. I. Abrikosova. Zh. Eksp Teor Fiz, 21:945, 1951.

[45] E.M. Lifshitz. The theory of molecular attractive forces between solids. Perspectives in Theoretical Physics, 2(1):329-349, 1956.

[46] I. E. Dzyaloshinskii and L. P. Pitaevskii. Van der Waals Forces in an Inhomogeneous Dielectric. J. Exptl. Theoret. Phys. (U.S.S.R.), 36(36):1797-1805, 1959.

[47] I.E. DZYALOSHINSKII, E.M. LIFSHITZ, L.P. PITAEVSKII, and D. ter Haar. Van der Waals forces in liquid films. Perspectives in Theoretical Physics, 37(1):425-441, 1960.

[48] Geoffrey Taylor and Proc R Soc Lond A. The dynamics of thin sheets of fluid II. Waves on fluid sheets. Proceedings of the Royal Society of London. Series A. Mathematical and Physical Sciences, 253(1274):296$312,1959$.

[49] D. R. Brown. A study of the behaviour of a thin sheet of moving liquid. Journal of Fluid Mechanics, 10(2):297-305, 1961.

[50] Malcom .B. Williams and Stephen H. Davis. Nonlinear Theory of Film Rupture. Journal of Colloid and Interface Science, 90(1), 1982.

[51] S. P. Lin, Z. W. Lian, and B. J. Creighton. Absolute and convective instability of a liquid sheet. Journal of Fluid Mechanics, 220(September):673689, 1990.

[52] M. P. Ida and M. J. Miksis. Thin film rupture. Applied Mathematics Letters, 9(3):35-40, 1996. 
[53] L. E. Scriven. Dynamics of a fluid interface Equation of motion for Newtonian surface fluids. Chemical Engineering Science, 12(2):98-108, 1960.

[54] Takuya Yamamoto, Yasunori Okano, and Sadik Dost. Development of a numerical model for marangoni convection in the micro-scale environment. Journal of Thermal Science and Technology, 11(3), 2016.

[55] Emmanouil Chatzigiannakis, Nick Jaensson, and Jan Vermant. Thin liquid films: Where hydrodynamics, capillarity, surface stresses and intermolecular forces meet. Current Opinion in Colloid and Interface Science, 53, 2021.

[56] A. Ponce-Torres, J. M. Montanero, M. A. Herrada, E. J. Vega, and J. M. Vega. Influence of the Surface Viscosity on the Breakup of a SurfactantLaden Drop. Physical Review Letters, 118(2):1-5, 2017.

[57] Bruna C. Leopércio. Kinetics of cyclopentane hydrate formation - an interfacial rheology study, 2016.

[58] Jürgen Krägel and Svetlana R. Derkatch. Interfacial shear rheology. Current Opinion in Colloid and Interface Science, 15(4):246-255, 2010.

[59] Gerald G. Fuller and Jan Vermant. Complex Fluid-Fluid Interfaces: Rheology and Structure. Annual Review of Chemical and Biomolecular Engineering, 3(1):519-543, 2012.

[60] Julia Maldonado-Valderrama and Juan M.Rodríguez Patino. Interfacial rheology of protein-surfactant mixtures. Current Opinion in Colloid and Interface Science, 15(4):271-282, 2010.

[61] John C Slattery, Leonard Sagis, and Eun-Suok Oh. Interfacial transport phenomena. Springer Science \& Business Media, 2007.

[62] M Boussinesq. Sur l'existence d'une viscosité superficielle, dans la mince couche de transition séparant un liquide d'un autre fluide contigue. Ann. Chim., 29:349-357, 1913.

[63] S. Gross and A. Reusken. Numerical simulation of continuum models for fluid-fluid interface dynamics. European Physical Journal: Special Topics, 222(1):211-239, 2013.

[64] O. E. Jensen and J. B. Grotberg. Insoluble surfactant spreading on a thin viscous film: Shock evolution and film rupture. Journal of Fluid Mechanics, 240:259-288, 1992. 
[65] A. De Wit, D. Gallez, and C. I. Christov. Nonlinear evolution equations for thin liquid films with insoluble surfactants. Physics of Fluids, 6(10):3256-3266, 1994.

[66] Sumeet Suresh Thete, Christopher Anthony, Osman A. Basaran, and Pankaj Doshi. Self-similar rupture of thin free films of power-law fluids. Physical Review E - Statistical, Nonlinear, and Soft Matter Physics, 92(2):2-6, 2015.

[67] S. Thete, C. Anthony, M. Harris, O. Basaran, and P. Doshi. Selfsimilarity and scaling transitions during rupture of thin free films of Newtonian fluids. Physics of Fluids, 28(9), 2016.

[68] Patrick T. Underhill, Amir H. Hirsa, and Juan M. Lopez. Modelling steady shear flows of Newtonian liquids with non-Newtonian interfaces. Journal of Fluid Mechanics, 814:5-23, 2017.

[69] M. Saad Bhamla, Chew Chai, Noelle I. Rabiah, John M. Frostad, and Gerald G. Fuller. Instability and breakup of model tear films. Investigative Ophthalmology and Visual Science, 57(3):949-958, 2016.

[70] Alireza Mohammad Karim, Wieslaw J. Suszynski, William B. Griffith, Saswati Pujari, Lorraine F. Francis, and Marcio S. Carvalho. Effect of rheological properties of shear thinning liquids on curtain stability. Journal of Non-Newtonian Fluid Mechanics, 263(November 2018):6976,2019 .

[71] Alireza Mohammad Karim, Wieslaw J. Suszynski, Saswati Pujari, Lorraine F. Francis, and Marcio S. Carvalho. Delaying breakup and avoiding air entrainment in curtain coating using a two-layer liquid structure. Chemical Engineering Science, 213:115376, 2020.

[72] Lou Kondic, Alejandro G. Gonzalez, Javier A. Diez, Jason D. Fowlkes, and Philip Rack. Liquid-State Dewetting of Pulsed-Laser-Heated Nanoscale Metal Films and Other Geometries. Annual Review of Fluid Mechanics, 52:235-262, 2020.

[73] D. Moreno-Boza, A. Martínez-Calvo, and A. Sevilla. The role of inertia in the rupture of ultrathin liquid films. Physics of Fluids, 32(11), 2020.

[74] Alejandro G. González, Javier A. Diez, and Mathieu Sellier. Inertial and dimensional effects on the instability of a thin film. Journal of Fluid Mechanics, 787(i):449-473, 2015. 
[75] Bruno M. Kassar. Numerical simulation of multiphase flows with enhanced curvature computation by point-clouds sampling, 2016.

[76] John W. Cahn and John E. Hilliard. Free energy of a nonuniform system. i. interfacial free energy. The Journal of Chemical Physics, 28(2):258$267,1958$.

[77] Bing Dai and L. Gary Leal. The mechanism of surfactant effects on drop coalescence. Physics of Fluids, 20(4), 2008.

[78] Tayfun E. Tezduyar. Interface-tracking and interface-capturing techniques for finite element computation of moving boundaries and interfaces. Computer Methods in Applied Mechanics and Engineering, 195(2324):2983-3000, 2006.

[79] Salih Ozen Unverdi and Grétar Tryggvason. A front-tracking method for viscous, incompressible, multi-fluid flows. Journal of Computational Physics, 100(1):25-37, 1992.

[80] Francis H. Harlow and J. Eddie Welch. Numerical calculation of timedependent viscous incompressible flow of fluid with free surface. Physics of Fluids, 8(12):2182-2189, 1965.

[81] Bart J. Daly. Numerical study of two fluid rayleigh-taylor instability. Physics of Fluids, 10(2):297-307, 1967.

[82] Soyoung You and Klaus Jürgen Bathe. Transient solution of 3D free surface flows using large time steps. Computers and Structures, 158:346$354,2015$.

[83] A. Prosperetti and G. Tryggvason. Polygon Mesh Processing. Cambridge University Press, 2007.

[84] O. Ubbink and R.I. Issa. A method for capturing sharp fluid interfaces on arbitrary meshes. Journal of Computational Physics, 153(1):26-50, 1999.

[85] Vinay R. Gopala and Berend G.M. van Wachem. Volume of fluid methods for immiscible-fluid and free-surface flows. Chemical Engineering Journal, 141(1):204-221, 2008.

[86] J. Klostermann, K. Schaake, and R. Schwarze. Numerical simulation of a single rising bubble by vof with surface compression. International Journal for Numerical Methods in Fluids, 71(8):960-982, 2013. 
[87] J. Donea, S. Giuliani, and J. P. Halleux. An arbitrary lagrangian-eulerian finite element method for transient dynamic fluid-structure interactions. Computer Methods in Applied Mechanics and Engineering, 33(1-3):689$723,1982$.

[88] Joan Baiges, Ramon Codina, Arnau Pont, and Ernesto Castillo. An adaptive Fixed-Mesh ALE method for free surface flows. Computer Methods in Applied Mechanics and Engineering, 313:159-188, 2017.

[89] C. W. Hirt, A. A. Amsden, and J. L. Cook. An arbitrary LagrangianEulerian computing method for all flow speeds. Journal of Computational Physics, 14(3):227-253, 1974.

[90] Thomas J.R. Hughes, Wing Kam Liu, and Thomas K. Zimmermann. Lagrangian-Eulerian finite element formulation for incompressible viscous flows. Computer Methods in Applied Mechanics and Engineering, 29(3):329-349, 1981.

[91] M. Souli and J. P. Zolesio. Arbitrary Lagrangian-Eulerian and free surface methods in fluid mechanics. Computer Methods in Applied Mechanics and Engineering, 191(3-5):451-466, 2001.

[92] Amaresh Sahu, Yannick A.D. Omar, Roger A. Sauer, and Kranthi K. Mandadapu. Arbitrary Lagrangian-Eulerian finite element method for curved and deforming surfaces: I. General theory and application to fluid interfaces. Journal of Computational Physics, 407:109253, 2020.

[93] S. Tanaka and K. Kashiyama. ALE finite element method for FSI problems with free surface using mesh re-generation method based on background mesh. International Journal of Computational Fluid Dynamics, 20(3-4):229-236, 2006.

[94] Sashikumaar Ganesan and Lutz Tobiska. A coupled arbitrary Lagrangian-Eulerian and Lagrangian method for computation of free surface flows with insoluble surfactants. Journal of Computational Physics, 228(8):2859-2873, 2009.

[95] Ivan Fumagalli, Nicola Parolini, and Marco Verani. On a free-surface problem with moving contact line: From variational principles to stable numerical approximations. Journal of Computational Physics, 355(03):253-284, 2018.

[96] Ye Wang, Jiejin Cai, Qiong Li, Huaqiang Yin, and Xingtuan Yang. Diffuse interface simulation of bubble rising process: a comparison of 
adaptive mesh refinement and arbitrary lagrange-euler methods. Heat and Mass Transfer/Waerme- und Stoffuebertragung, 54(6):1767-1778, 2018.

[97] Martin S Alnæs, Jan Blechta, Johan Hake, August Johansson, Benjamin Kehlet, Anders Logg, Chris Richardson, Johannes Ring, E Rognes, and Garth N Wells. The FEniCS Project Version 1 . 5. 3(100):9-23, 2015.

[98] Guido van Rossum and The Python Development Team. The Python Language Reference - Release 3.8.1. 2018.

[99] P. M. Gresho and R. L. Sani. Incompressible Flow and the Finite Element Method. John Wiley and Sons, 1998.

[100] Marcio da Silveira Carvalho and Juliana Vianna Valério. Introdução ao método de Elementos Finitos: aplicação em dinâmica dos fluidos. 2012.

[101] Cécile Daversin-Catty, Chris N. Richardson, Ada J. Ellingsrud, and Marie E. Rognes. Abstractions and automated algorithms for mixed domain finite element methods. arXiv, 2019.

[102] R.A. Frazer, W.J. Duncan, and A.R. Collar. Elementary matrices and some applications to dynamics and differential equations. 1937.

[103] Cornelis B. Biezeno and Richard Grammel. Technische Dynamik. 1953.

[104] Boris G. Galerkin. Series occurring in various questions concerning the elastic equilibrium of rods and plates. Engineers Bulletin (Vestnik Inzhenerov), 19:897-908 (in Russian), 1915.

[105] J Donea, Antonio Huerta, and JP Ponthot. Chapter 14: Arbitrary Lagrangian-Eulerian Methods. Encyclopedia of Computational, pages $1-25,2004$.

[106] W.J. Gordon and C.H. Hall. Construction of curvilinear co-ordinate systems and applications to mesh generation. Int. J. Numer. Methods Eng., 7(4):461-477, 1973.

[107] A.M. Winslow. Equipotential zoning of two-dimensional meshes. Report UCRL-7312, 1963.

[108] S. Giuliani. An algorithm for continuous rezoning of the hydrodynamic grid in arbitrary Lagrangian-Eulerian computer codes. Nucl. Eng. Des., 72:205-212, 1982. 
[109] H. Askes and A. Rodrigues-Ferran. A combined rh-adaptative scheme based on dumain subdivision. Formulation and linear examples. Int. J. Numer. Methods Eng., 51(3):253-273, 2001.

[110] R. Courant, H. Lewy, and K. Friedrichs. Über die partiellen Differenzengleichungen der mathematischen Physik. Mathematische Annalen, 100(1):32-74, 1928.

[111] R. Courant, H. Lewy, and K. Friedrichs. On the partial difference equations of mathematical physics. AEC Research and Development Report, 1956. 